Old Dominion University

ODU Digital Commons

Electrical \& Computer Engineering Theses \&

Dissertations

Fall 1990

\title{
The Modeling of the Interactions of Pellets With Magnetic Fusion Plasmas for Alpha Particle Diagnostics
}

Abdel-Ghafar Mahmood El-Cashlan

Old Dominion University

Follow this and additional works at: https://digitalcommons.odu.edu/ece_etds

Part of the Electrical and Computer Engineering Commons

\section{Recommended Citation}

El-Cashlan, Abdel-Ghafar M.. "The Modeling of the Interactions of Pellets With Magnetic Fusion Plasmas for Alpha Particle Diagnostics" (1990). Doctor of Philosophy (PhD), Dissertation, Electrical \& Computer Engineering, Old Dominion University, DOI: 10.25777/gnav-3h08

https://digitalcommons.odu.edu/ece_etds/173

This Dissertation is brought to you for free and open access by the Electrical \& Computer Engineering at ODU Digital Commons. It has been accepted for inclusion in Electrical \& Computer Engineering Theses \& Dissertations by an authorized administrator of ODU Digital Commons. For more information, please contact digitalcommons@odu.edu. 
THE MODELING OF THE INTERACTIONS OF PELLETS WITH MAGNETIC FUSION

PLASMAS FOR ALPHA PARTICLE DIAGNOSTICS

by

Abdel-Ghafar Mahmood El-Cashlan

M.SC. 1982, University of Helwan, Cairo, EGYPT

A Dissertation Submitted to the Faculty of Old Dominion University in Partial Fulfillment of the Requirements for the Degree of

DOCTOR OF PHILOSOPHY

ELECTRICAL ENGINEERING

OLD DOMINION UNIVERSITY

FALL 1990

Approved by:

Glenn A. Gerdin (Director)

Linda L. Vahala (Director) 


\title{
ABSTRACT \\ The Modeling of the Interactions of Pellets with Magnetic Fusion Plasmas for Alpha Particle Diagnostics
}

\author{
Abdel-Ghafar Mahmood El-Cashlan \\ Old Dominion University \\ Director(s): Dr. Glenn A Gerdin \\ Dr. Linda L. Vahala
}

\begin{abstract}
A model for the interactions of hydrogen and carbon pellets with thermonuclear fusion plasmas has been developed and solved to determine the charge-state spatial distributions in the ablation cloud surrounding the pellet. The ionization is treated in a self-consistent manner in the energy balance and the collisional radiative (CR) model is used to calculate the ionization levels. External plasma electron effects on ionization are included. The electron heat flux is modeled as a multigroup distribution. The channelling of the flow along the field line is treated phenomenologically by restricting the cross-sectional area of the flow. The predictions of the model are compared with the results from the General Atomics experiments on the TEXT tokamak for $\mathrm{C}+2$ and $\mathrm{C}+3$ clouds of definite size. A reasonable agreement for the $\mathrm{C}+2$ cloud is found.
\end{abstract}




\section{ACKNOWLEDGEMENTS}

This work was made possible with contribution from a number of people to whom I am very grateful. At the outset I would like to express my deepest gratitude to Dr. Glenn Gerdin, the chair of my dissertation committee, for the insight he provided throughout the course of this research, and to Dr. Linda Vahala, the co-chair of the committee, for guidance and encouragement. I would like to thank the other committee members, Dr. Amin Dharamsi and Dr. James Cox, Jr., for their guidance and thorough review of the manuscript. I am grateful to Drs. Raymond Fisher and Paul Parks of the General Atomics Corporation, for providing the experimental data and for their suggestions on the theoretical model.

I would also like to record my deepest thanks to Dr. Hasan Erkaya and Mr. Zayn El-Abdean for their great help during the preparation of this dissertation. Finally, my most loving gratitude goes to my parents, Mahmood and Wafiqah, my wife, Amal, and my daughters, Eshraq and Marwah, for their great support without which my graduate studies would not have been completed. 
TABLE OF CONTENTS

$\underline{\text { PAGE }}$

ACKNOWLEDGEMENTS $\ldots \ldots \ldots \ldots \ldots \ldots \ldots \ldots \ldots \ldots$ ii

TABLE OF CONTENTS $\ldots \ldots \ldots \ldots \ldots \ldots \ldots \ldots \ldots \ldots \ldots \ldots \ldots \ldots \ldots$ iii

LIST OF TABLES $\ldots \ldots \ldots \ldots \ldots \ldots \ldots \ldots \ldots \ldots \ldots \ldots \ldots \ldots \ldots \ldots$

LIST OF FIGURES $\ldots \ldots \ldots \ldots \ldots \ldots \ldots \ldots \ldots \ldots \ldots \ldots \ldots \ldots \ldots \ldots \ldots$ vii

CHAPTER

1. INTRODUCTION $\ldots \ldots \ldots \ldots \ldots \ldots \ldots \ldots \ldots \ldots \ldots \ldots$

1.1 BACKGROUND AND MOTIVATION FOR THIS STUDY 1

1.2 BRIEF REVIEW OF PREVIOUS PERTINENT WORK . . . 2

1.3 OBJECTIVES AND SCOPE OF THE STUDY $\ldots \ldots \ldots 4$

2. EQUILIBRIUM AND NONEQUILIBRIUM THEORY $\ldots \ldots \ldots \ldots 6$

$2.1 \quad$ INTRODUCTION $\ldots \ldots \ldots \ldots \ldots \ldots \ldots \ldots \ldots, 6$

2.2 PLASMA MODELS $\ldots \ldots \ldots \ldots \ldots \ldots \ldots \ldots \ldots, 7$

2.3 THE RATE EQUATIONS $\ldots \ldots \ldots \ldots \ldots \ldots \ldots, 9$

2.4 CRITERION FOR EQUILIBRIUM $\ldots \ldots \ldots \ldots \ldots \ldots, 12$

3. THE GENERAL MODEL FORMULATION $\ldots \ldots \ldots \ldots \ldots \ldots, 17$

$3.1 \quad$ INTRODUCTION . . . . . . . . . . . . . . . . . . 17

3.2 GENERAL ASSUMPTIONS $\ldots \ldots \ldots \ldots \ldots \ldots \ldots \ldots, 17$

3.3 GOVERNING EQUATIONS . . . . . . . . . . . . . . 19

3.4 THE GENERAL EQUILIBRIUM MODEL . . . . . . . 24

3.5 METHOD OF SOLUTION $\ldots \ldots \ldots \ldots \ldots \ldots \ldots, 25$

iii 
TABLE OF CONTENTS - Continued

4. APPLICATION TO HYDROGEN PELLETS $\ldots \ldots \ldots \ldots \ldots 28$

$4.1 \quad$ INTRODUCTION . . . . . . . . . . . . . . . 28

4.2 HYDROGEN MODEL EQUATIONS $\ldots \ldots \ldots \ldots \ldots \ldots 28$

4.3 INITIAL CONDITIONS $\ldots \ldots \ldots \ldots \ldots \ldots \ldots \ldots, 33$

4.4 DISCUSSION OF RESULTS $\ldots \ldots \ldots \ldots \ldots \ldots \ldots \ldots, 35$

5. APPLICATION TO CARBON PELLETS $\ldots \ldots \ldots \ldots \ldots \ldots .47$

$5.1 \quad$ INTRODUCTION $\ldots \ldots \ldots \ldots \ldots \ldots \ldots \ldots \ldots \ldots$

5.2 HEAT FLUX EQUATION ................. 49

5.3 CARBON PELLET MODELS $\ldots \ldots \ldots \ldots \ldots \ldots \ldots 49$

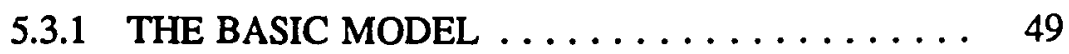

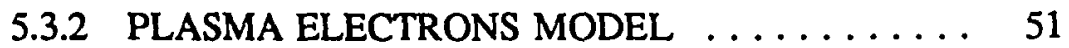

5.3.3 CHANNEL FLOW MODEL ........... 54

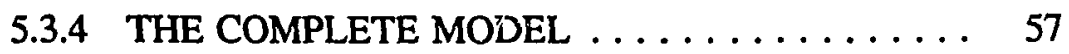

5.4 INTTIAL CONDITIONS $\ldots \ldots \ldots \ldots \ldots \ldots \ldots \ldots . \ldots \ldots$

5.5 SHOCK EQUATION $\ldots \ldots \ldots \ldots \ldots \ldots \ldots \ldots \ldots \ldots \ldots$

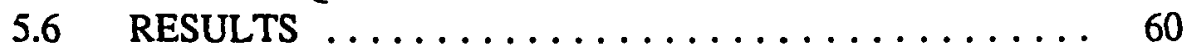

6. COMPARISON WITH EXPERIMENTAL RESULTS $\ldots \ldots \ldots, 72$

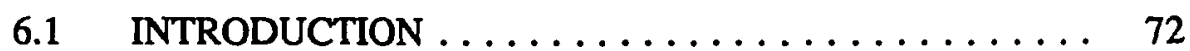

6.2 CRITICAL FLOW MODEL . ............. 72

6.3 COMPARISON OF THE MODEL PREDICTIONS $\ldots \ldots .74$

6.4 PELLET PENETRATION $\ldots \ldots \ldots \ldots \ldots \ldots \ldots, 76$

7. CONCLUSIONS AND RECOMMENDATIONS $\ldots \ldots \ldots \ldots \ldots$

$7.1 \quad$ CONCLUSIONS ....................... 86

7.2 RECOMMENDATIONS $\ldots \ldots \ldots \ldots \ldots \ldots \ldots \ldots 87$

LIST OF REFERENCES $\ldots \ldots \ldots \ldots \ldots \ldots \ldots \ldots \ldots \ldots \ldots$ 
TABLE OF CONTENTS - Continued

\section{APPENDICES}
A. DERIVATION OF THE EXPRESSIONS $U_{1}, U_{2}, U_{3}$, AND $U_{4} \ldots \ldots 93$
B. DERIVATION OF THE HEAT FLUX DEGRADATION ...... 101
C. DERIVATION OF IONIZATION RATE $\ldots \ldots \ldots \ldots \ldots \ldots \ldots$
D. DERIVATION OF THE EXPRESSIONS $U_{1}{ }^{X}, U_{2}{ }^{x}, U_{3}{ }^{X}$, AND $U_{4}{ }^{x} .107$
E. SHOCK EQUATIONS $\ldots \ldots \ldots \ldots \ldots \ldots \ldots \ldots \ldots \ldots \ldots \ldots$
F. CRITICAL FLOW MODEL ................ 119 


\section{LIST OF TABLES}

\section{TABLE}

PAGE

1-1 The ratio $\xi_{\mathrm{j}} / \mathrm{r}_{\mathrm{p}}$ for TEXT, TFTR and CIT devices $\ldots \ldots \ldots \ldots$

4-1 $\xi_{\mathrm{j}} / \mathrm{r}_{\mathrm{p}}$ ratio for $\mathrm{n}_{c}=10^{14} \mathrm{~cm}^{-3} \ldots \ldots \ldots \ldots \ldots \ldots \ldots \ldots \ldots \ldots$

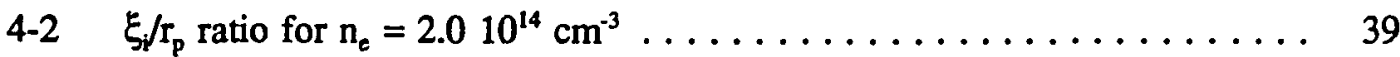

4-3 $\xi_{\mathrm{j}} / \mathrm{r}_{\mathrm{p}}$ ratio for $\mathrm{n}_{c}=3.010^{14} \mathrm{~cm}^{-3} \ldots \ldots \ldots \ldots \ldots \ldots \ldots$

4-4 The minimum background electron temperature $T_{e}$ for $\xi_{j} / r_{p} \leq 1.0 \ldots \ldots 41$

4-5 The minimum background electron temperature $T_{e}$ for $\xi_{j} / r_{p} \leq 0.1 \ldots 42$

4-6 The minimum background electron temperature $T_{c}$ for $\xi_{/} / r_{p} \leq 0.01 \ldots 43$

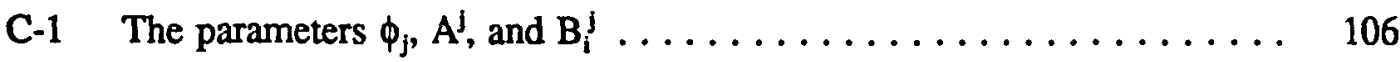




\section{LIST OF FIGURES}

\section{FIGURE}

2-1 Geometry of cloud. Alpha particles will interact with an ablation cloud surrounding an injected pellet. By observing helium neutrals, information on the alpha particles can be obtained. . . . . . . . . . . .

4-1 Comparison of Mach number $M$, flow velocity $v$, total cloud number density $n$, cloud temperature $T$, and ionization $\mathrm{f} 1$ for equilibrium and non-equilibrium hydrogen models. The pellet radius is $0.28 \mathrm{~cm}$, and the background plasma temperature and density are $11.5 \mathrm{keV}$ and $1.01 \times 10^{14}$ $\mathrm{cm}^{-3}$, respectively. $\mathrm{r}_{*}=0.484 \mathrm{~cm}$ and $\mathrm{v}_{*}=1.72 \times 10^{6} \mathrm{~cm} / \mathrm{s} . \ldots \ldots \ldots$

4-2 Comparison of Mach number $M$, flow velocity $v$, total cloud number density $n$, cloud temperature $T$, and ionization $f 1$ for equilibrium and non-equilibrium hydrogen models. The pellet radius is $0.2 \mathrm{~cm}$, and the background plasma temperature and density are $5 \mathrm{keV}$ and $1.0 \times 10^{14} \mathrm{~cm}^{-3}$, respectively. $r_{*}=0.34 \mathrm{~cm}$ and $v_{*}=1.6 \times 10^{6} \mathrm{~cm} / \mathrm{s} . \ldots \ldots \ldots \ldots$

4-3 Comparison of Mach number $M$, flow velocity $v$, total cloud number density $n$, cloud temperature $T$, and ionization $\mathrm{f} 1$ for equilibrium and non-equilibrium hydrogen models. The pellet radius is $0.25 \mathrm{~cm}$, and the background plasma temperature and density are $6.33 \mathrm{keV}$ and $1.0 \times 10^{14}$ $\mathrm{cm}^{-3}$, respectively. $r_{*}=0.427 \mathrm{~cm}$ and $v_{*}=1.7 \times 10^{6} \mathrm{~cm} / \mathrm{s} . \ldots \ldots \ldots$

5-1 Comparison of a) Mach number $M$, flow velocity $v$, and cloud temperature $T, b)$ total cloud number density $n$ and charge-state fractions ( $f 1, f 2, f 3, f 4$ ) for equilibrium and non-equilibrium basic models. The pellet radius is $0.05 \mathrm{~cm}$, and the background plasma temperature and density are $20.0 \mathrm{keV}$ and $1.0 \times 10^{14} \mathrm{~cm}^{-3}$, respectively. $r_{0}=0.067 \mathrm{~cm}$ and $v_{*}=5.3 \times 10^{5} \mathrm{~cm} / \mathrm{s}$.

5-2 Comparison of a) Mach number $M$, flow velocity $v$, and cloud temperature $T, b)$ total cloud number density $n$ and charge-state fractions ( $f 1, f 2, f 3, f 4$ ) for equilibrium and non-equilibrium basic models. The pellet radius is $0.05 \mathrm{~cm}$, and the background plasma temperature and density are $10.0 \mathrm{keV}$ and $1.0 \times 10^{14} \mathrm{~cm}^{-3}$, respectively. $\mathrm{r}_{*}=0.067 \mathrm{~cm}$ and $\mathrm{v}_{*}=5.31 \times 10^{5} \mathrm{~cm} / \mathrm{s}$. . 


\section{LIST OF FIGURES - CONTINUED}

5-3 Comparison of a) Mach number $M$, flow velocity $v$, and cloud temperature $T, b)$ total cloud number density $n$ and charge-state fractions ( $f 1, f 2, f 3, f 4$ ) for equilibrium and non-equilibrium basic models. The pellet radius is $0.025 \mathrm{~cm}$, and the background plasma temperature and density are $3.0 \mathrm{keV}$ and $5.0 \times 10^{13} \mathrm{~cm}^{-3}$, respectively. $r_{*}=0.033 \mathrm{~cm}$ and $v_{\bullet}=4.38 \times 10^{5} \mathrm{~cm} / \mathrm{s} .$.

5-4 The predictions of the basic model of the Mach number $M$, flow velocity $v$, total cloud number density $n$, cloud temperature $T$, and charge-state fractions ( $\mathrm{f} 1, \mathrm{f} 2, \mathrm{f3}, \mathrm{f4})$. The pellet radius is $0.05 \mathrm{~cm}$, and the background plasma temperature and density are $20.0 \mathrm{keV}$ and $1.0 \times 10^{14} \mathrm{~cm}^{-3}$,

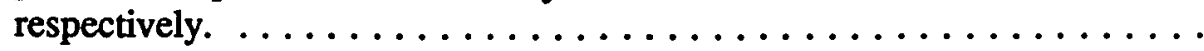

5-5 The predictions of the plasma electrons model of the Mach number $M$, flow velocity $v$, total cloud number density $n$, cloud temperature $T$, and charge-state fractions ( $\mathrm{f} 1, \mathrm{f} 2, \mathrm{f} 3, \mathrm{f} 4)$. The pellet radius is $0.05 \mathrm{~cm}$, and the background plasma temperature and density are $20.0 \mathrm{keV}$ and $1.0 \times 10^{14}$

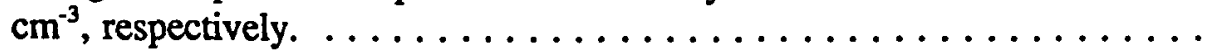

5-6 The predictions of the channel flow model of the Mach number M, flow velocity $v$, total cloud number density $n$, cloud temperature $T$, and charge-state fractions ( $\mathrm{fl}, \mathrm{f2}, \mathrm{f3}, \mathrm{f4}$ ). The pellet radius is $0.05 \mathrm{~cm}$, and the background plasma temperature and density are $20.0 \mathrm{keV}$ and $1.0 \times 10^{14}$ $\mathrm{cm}^{-3}$, respectively. The channel half-width is $25 \mathrm{r}_{*}$, and Eq. (5-6) is used

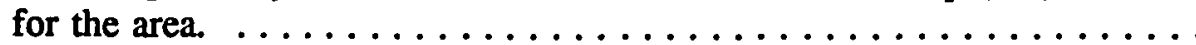

5-7 The predictions of the complete model of the Mach number M, flow velocity $v$, total cloud number density $n$, cloud temperature $T$, and charge-state fractions $(f 1, f 2, f 3, f 4)$. The pellet radius is $0.05 \mathrm{~cm}$, and the background plasma temperature and density are $20.0 \mathrm{keV}$ and $1.0 \times 10^{14}$ $\mathrm{cm}^{-3}$, respectively. The channel half-width is $25 \mathrm{r}_{*}$, and Eq. $(5-6)$ is used for the area. . . . . . . . . . . . . . . . . . . . . .

6-1 The predictions of the complete model and critical flow model for a typical TEXT case: a) charge state fraction and total number density, b) Mach number, flow velocity and cloud temperature. The pellet radius is $0.03 \mathrm{~cm}$, and the background plasma temperature and density are $1.0 \mathrm{keV}$ and $5.0 \times 10^{13} \mathrm{~cm}^{-3}$, respectively. The channel half-width is $20 \mathrm{r}_{*}$, and Eq. $(5-26)$ is used for the area. $r_{*}=0.038 \mathrm{~cm}$ and $v_{*}=4.93 \times 10^{5} \mathrm{~cm} / \mathrm{s} . \ldots$ 


\section{LIST OF FIGURES - CONTINUED}

6-2 The predictions of the complete model and critical flow model for a typical TEXT case. The pellet radius is $0.03 \mathrm{~cm}$, and the background plasma temperature and density are $1.0 \mathrm{keV}$ and $5.0 \times 10^{13} \mathrm{~cm}^{-3}$, respectively. The channel half-width is $20 \mathrm{r.n}$, and Eq. (5-26) is used for the area. $r_{*}=0.038 \mathrm{~cm}$ and $v_{*}=4.93 \times 10^{5} \mathrm{~cm} / \mathrm{s} . \ldots \ldots \ldots$

6-3 Comparison of the predictions of theory with the data from TEXT for $\mathrm{C}+2$. The pellet radius is $0.03 \mathrm{~cm}$ and the background plasma temperature

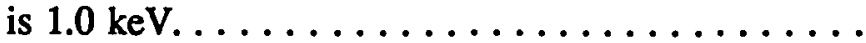

6-4 Comparison of the predictions of theory with the data from TEXT for $\mathrm{C}+3$. The pellet radius is $0.03 \mathrm{~cm}$ and the background plasma temperature is $1.0 \mathrm{keV} . \ldots \ldots \ldots \ldots \ldots \ldots \ldots \ldots$

6-5 The predictions of the complete model and critical flow model for a typical CIT case: a) charge state fraction and total number density, b) Mach number, flow velocity and cloud temperature. The pellet radius is $0.05 \mathrm{~cm}$, and the background plasma temperature and density are $20.0 \mathrm{keV}$ and $1.0 \times 10^{14} \mathrm{~cm}^{-3}$, respectively. The channel half-width is $20 \mathrm{r}$, and Eq. $(6-16)$ is used for the area. $r_{*}=0.067 \mathrm{~cm}$ and $v_{*}=5.3 \times 10^{5} \mathrm{~cm} / \mathrm{s} . \ldots \ldots$

6-6 The predictions of the complete model and critical flow morlel for a typical CIT case. The pellet radius is $0.05 \mathrm{~cm}$, and the background plasma temperature and density are $20.0 \mathrm{keV}$ and $1.0 \times 10^{14} \mathrm{~cm}^{-3}$, respectively. The channel half-width is $20 \mathrm{r}_{*}$, and Eq. (6-16) is used for the area. $r_{*}=0.067$

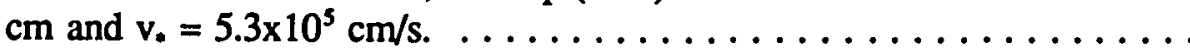

6-7 The penetration depth measured from plasma edge versus the pellet velocity. The pellet radius is $0.14 \mathrm{~cm}$, and the background tempreature and density profiles are of the form $T_{e}=T_{e 0}\left(1-x^{2}\right)$ and $n_{e}=n_{e 0}\left(1-x^{2}\right)$ with $\mathrm{T}_{\mathrm{e} 0}=16.7 \mathrm{keV}$, and $\mathrm{n}_{\mathrm{e} 0}=1.65 \times 10^{14} \mathrm{~cm}^{-3} \ldots \ldots \ldots \ldots \ldots$

6-8 Radius of a carbon pellet versus the normalized minor radius $(r / a)$. The pellet velocity is $10 \mathrm{~km} / \mathrm{s}$. Also shown are the preinjection profiles for the background temperature and density $\ldots \ldots \ldots \ldots \ldots \ldots$ 


\section{Chapter 1.}

\section{INTRODUCTION}

\subsection{Background and motivation for this study}

Heating a plasma to thermonuclear temperature has been and remains one of the principal objectives of controlled-fusion research. The fusion alpha particles, created in the deuterium-tritium (D-T) reactions, give up their energy to the plasma particles via Coulomb collisions, and thus provide an intrinsic self-heating mechanism once thermonuclear temperatures are achieved. Understanding the effect of plasma on alpha confinement and the effect of alphas on plasma confinement and heating requires information on the fast confined alpha-particle energy distribution. There have been several techniques proposed to diagnose the properties of alpha particles in a fusion plasma [1-9]. The technique of using pellet injection to provide a target for fast alpha particle interaction through charge exchange reaction is considered to be promising.

Once thermonuclear temperatures are achieved in a D-T plasma, the achievement of a prolonged plasma burn will depend on, among other things, the maintenance of the plasma ion density against depletion due to fusion and transport losses. Some mechanism for refueling the burning plasma is needed. Most magnetic fusion reactor concepts have incorporated pellet injection refueling schemes into their designs [10-22]. Therefore, modeling the pellet-plasma interaction is very important to both alpha particle diagnostics and refueling techniques. In both of these applications a clear understanding of the 
physics of the ablation cloud, surrounding an injected pellet into a hot magnetplasma, is required.

\subsection{Brief review of previous pertinent work}

A number of theories have been proposed to describe the interaction between hydrogenic pellet and magnetic fusion plasma. The most accepted theory is the neutral gas shielding (NGS) model [12]. The NGS model is a 1-D spherically symmetric quasisteady description of the pellet ablating fluid. According to this model, hot electrons of ambient plasma bombarding the pellet surface build up a neutral gas cloud around it on very short time scale. The incident electron heat flux deposits its energy in the ablation cloud through elastic and inelastic collisions with hydrogen molecules. The thickness of this cloud is of self-regulating nature; if the cloud gets thinner, ablation increases causing the cloud to grow and vice-versa. The ablatant leaves the pellet surface slowly and then accelerates to supersonic velocity. Far from the pellet surface, subsonic flow is restored. Atomic processes and magnetic field effects are ignored in this NGS model. Parks and Tumbull solved the fluid equations for the NGS model describing the gas dynamic expansion of an inviscid perfect gas with constant specific heats. Scaling laws for hydrogen pellet ablation rate and ablation cloud properties were given.

The thermal dissociation and ionization process in the ablation cloud act as energy sinks, and consequently they slow the ablation rate and modify the cloud profile. A revised version $[17,18]$ of the NGS model included a self-consistent treatment of these atomic processes (ionization, dissociation) which occur in the ablation cloud. In this model atomic processes are governed by the Saha equation, since local thermodynamic 
equilibrium (LTE) is assumed. For low temperature background plasma, this model predicts singularities in the supersonic region of the flow [18]. These occur in the region where LTE is no longer valid. Thus it appears that a modification of this model to include non-LTE is necessary.

Since we are modeling the charge-state distribution in the cloud and since the ablatant is constrained to flow along the magnetic field once ionized, it is important to consider the interaction between the ablation flow and the magnetic field. The NGS model has been upgraded by Parks [19] to include the magnetic field effects. In his model, the cloud expansion is basically spherical out to a critical radius where the cloud pressure is equal to the magnetic field pressure. Then the expansion of the ionized position of the cloud changes from that of a spherical flow to a channel flow along the magnetic field lines. It has been shown that the ablation flow distorts magnetic field near the pellet surface. This distortion causes a modest reduction in ablation rate. The magnetic shielding of the pellet will reduce the required injection velocities and allow small pellet injection.

Recently, the injection of a low- $\mathrm{Z}$ pellet like $\mathrm{Li}, \mathrm{Be}$, and $\mathrm{C}$ is being studied for the purpose of plasma diagnostic applications [2-6]. For example, as a fast alpha-particle diagnostic tool, the high-density ablation cloud serves as the target material for doublecharge exchange reactions between the alphas and the cloud ions [2]. The difference in the ablation mechanism between a hydrogen pellet and a low- $Z$ pellet is due to the difference in their sublimation energy. Due to the low sublimation energy of frozen hydrogen, shielding is complete in hydrogen pellet ablation, and the heat flux at the pellet 
surface is essentially reduced to zero. For a low-Z pellet, the ablation cloud is more tenuous, and the heat flux arriving at the pellet surface is $30 \%$ to $70 \%$ of its asymptotic value [6].

More recently, Parks et.al. [6] developed a model for low-Z pellets. Their approach and assumptions are similar to those of the hydrogen pellet model [17], but this model does not provide a solution to the fluid equations. However, it establishes the sonicsurface variables in a self-consistent manner. The model is based on the Saha equation, , and therefore it is assumed that collisional processes dominate both ionization and recombination. The critical density at which LTE breaks down increases with $Z$, hence large regions of the flow will not satisfy LTE. The outer regions of the cloud are important for alpha particle diagnostics. Therefore, the inclusion of the non-LTE effect in the low-Z pellet model seems essential.

The charge-state-equilibrium model, where non-LTE effects (radiative and dielectronic recombination) are included, demands that the characteristic time for readjustment by collisions, $\tau_{i}$, be negligible compared with the time required for a fluid element, $\tau_{f}$, to encounter a significant change in conditions (temperature and density) as it moves through the flow field. If, as can happen in the lower density outer regions of the cloud, $\tau_{\mathrm{i}}$ is of the same order as $\tau_{\mathrm{f}}$, then the non-equilibrium effects must be taken into account $[23,24]$.

\subsection{Objectives and scope of the study}

The objective of the present study is to model the charge-state distributions for carbon pellets interacting with plasmas of temperature and density relevant to those of the 
Compact Ignition Tokamak (CIT) or the International Thermonuclear Experimental Reactor (TTER).

The scope of this study includes:

1. Charge state equilibrium model

2. Nonequilibrium flow model

3. Effects of external plasma electrons on the ablation cloud and channel flow

4. Application to hydrogen and carbon pellets

The organization of the present dissertation is as follows. A review of the equilibrium and non-equilibrium theories is given in chapter 2 . The basic assumptions and formulation of the physical model and computational techniques are given in chapter 3. The application of this model to hydrogen and carbon pellets is described in chapters 4 and 5, respectively. Correlation between this theory and previous experimental results is given in chapter 6. Summary of the conclusions and recommendations for further work are given in chapter 7 . 


\section{Chapter 2.}

\section{EQUILIBRIUM AND NONEQUILIBRIUM THEORY}

\subsection{Introduction}

In calculating the distribution of the atomic species among their various stages of ionization, conditions in the plasma can be considered to be at either of two extremes; (1) low-temperature and high-density near the pellets' surface where the assumption of local thermodynamic equilibrium (LTE) is valid (in which case 3-body collisions dominate the state populations) or (2) high temperature and low density far from the pellets' surface where the non-LTE, coronal equilibrium, is valid. In the latter case the 2-body radiative effects are the dominant mechanisms, of recombination and deexcitation. However, a number of situations exist in which the plasma can migrate between these two extremes, thus requiring a solution in which both 2-body and 3-body atomic processes are fully considered. Examples of this sort include stellar atmospheres, inertial confinement fusion target chambers, and the ablation cloud resulting from pellet injection into a magnetoplasma. The latter, of course, is the subject of this dissertation. Atomic processes have a significant effect on the macroscopic nature of the flow field in the ablation cloud $[17,18]$ and so must be considered in the modeling of this flow field. These processes are also crucial to alpha-particle diagnostics, since the charge-state distributions are required to compare with experiments. The complete set of fluid and rate equations is presented 
in chapter 3 . In this chapter we establish a criterion to characterize the flow as it departs from equilibrium toward non-equilibrium.

\subsection{Plasma Models}

Three types of model of a plasma are usually considered: (1) the coronal model in the limit of low density, (2) the model of local thermodynamic equilibrium (LTE) at high density, and (3) the model of a plasma in the intermediate region which is often referred to as the collisional-radiative model.

In the local thermodynamic equilibrium model (LTE), it is assumed that collisional processes dominate both ionization and recombination and that the charge states depend on local temperature and density of the plasma. The plasma conditions required for this to happen have been calculated and give rise to criteria for LTE to be a valid description of the plasma. The LTE criterion for electron density is given by [25]:

$$
n_{e}>1.7210^{14} T_{e}(e v)^{1 / 2} x(p, q)^{3} \mathrm{~cm}^{-3}
$$

where $x(p, q)$ is the excitation potential in $\mathrm{eV}$ between levels $\mathrm{p}$ and $\mathrm{q}$. For a given ion to be in LTE this inequality must be met by all pairs of levels of the ion. Since it is least met by the pair having the greatest excitation potential it is sufficient if the criterion is satisfied by this largest value of $x(p, q)$ for the ion.

In the previous studies $[17,18]$ LTE was assumed, hence the charge state distribution was obtained by the well-known Saha equation [26]. The advantage of this equation is that it is independent of atomic cross-sections. In the outer regions of the 
ablatant, however, the density is much too low to guarantee the validity of LTE. Therefore, we need to consider other models that meet the physics of our problem.

The atomic process models are based on solutions of the statistical balance equations where the dominant terms are not the inverse of each other. The steady-state coronal model is based on this argument, and its quantitative interpretation depends critically on atomic cross-sectional data [25]. Instead of each collisional process being balanced by its inverse as in the LTE model, the balance in the coronal model is between collisional ionization (and excitation) and radiative recombination (and spontaneous decay). There is a maximum electron density limit above which the coronal model breaks down [25]:

$$
n_{e}<6.0310^{10}(z+1)^{6} T_{e}(e V)^{1 / 2} \exp \left[(z+1)^{2} / T_{a}\right] c m^{-3}
$$

where $\mathrm{z}$ is the ionic charge.

For ccmpleteness, it is assumed in this model that if there is a change in the plasma parameters, it takes place sufficiently slowly for the population densities to take up their new steady state values at each instant. If there is a sudden change in the plasma parameters, the time dependent coronal model should be used [25].

For carbon pellet and $1 \mathrm{Mev}$ alpha particles, the most suitable cloud thickness [see Fig. (2-1)] has been shown to be in the range of $10^{17} / \mathrm{cm}^{2}$ to $10^{19} / \mathrm{cm}^{2}$ [1]. For pellet ablation clouds, this region is encountered in the portion of the flow where $\mathrm{C}^{+4}$ exist. If we apply both the LTE and coronal limits in this region we find that both criteria break down. Therefore, both 2-body and 3-body atomic processes must be simultaneously considered in calculating the charge state distribution. These processes are included in the 
collisional-radiative (CR) model [27-30] which we apply in this study. In the CR model, the populations of the atomic levels are determined by all collisional and radiative processes. The processes that can be included in calculations are the following: collisional excitation and de-excitation, ionization and the inverse process of three-body recombination, radiative and dielectronic recombination, and spontaneous radiative decay. When the degree of ionization is not too low, only the electronic collisions are of importance.

Following Magill [31], only the ground state of the ionization stages present in the plasma will be treated. Therefore, the atomic processes considered here are collisional ionization, radiative recombination, dielectronic recombination, and collisional recombination.

\subsection{The Rate Equations}

The number of ions in the $\mathrm{jth}$ ionization state, $\mathrm{n}_{\mathrm{j}}$, is determined from the coupled set of rate equations:

$$
d n_{j} / d t=n_{a L}\left[n_{j-1} C_{j-1}^{\text {coll }}-n_{j}\left(C_{j}^{\text {coll }}+\alpha_{j}^{\infty o g}\right)+n_{j+1} \alpha_{j+1}^{\infty \alpha t}\right] \quad j=1, Z
$$

where $\mathrm{n}_{\mathrm{eL}}$ is the ablatant electron density, $\mathrm{Z}$ is the atomic number, $\mathrm{C}_{\mathrm{j}}^{\mathrm{coll}}$ is the collisional ionization rate from state $\mathrm{j}$ to $\mathrm{j}+1$, and $\alpha_{\mathrm{j}+1}{ }^{\text {tot }}$ is the sum of the collisional, radiative, and dielectronic recombination rates from state $j+1$ to $j$.

Under steady state conditions, $\mathrm{d}_{\mathrm{j}} / \mathrm{dt}=0$, and the ionizations fractions, $\mathrm{f}_{\mathrm{j}}$, are determined by, 


$$
f_{j}=\left(\prod_{m=0}^{j-1} R_{m, m+1}\right) /\left(1+\sum_{l=1}^{Z} \prod_{m=0}^{l-1} R_{m, m+1}\right)
$$

where

$$
R_{m, m+1}=\frac{C_{m}^{\text {coll }}}{\alpha_{m+1}^{\text {coll }}+\alpha_{m+1}^{\text {rod }}+\alpha_{m+1}^{d \text { dil }}}
$$

Collisional recombination is a 3-body process involving two electrons and one ion. Radiative and dielectronic recombination are 2-body processes in which the excess energy is carried away by a photon as opposed to a second electron. Because of its stronger dependence on the electron density, collisional effects dominate the recombination process in the high density limit.

Collisional ionization involves the reaction $\mathrm{A}^{j+}+\mathrm{e}^{-} \rightarrow \mathrm{A}^{(j+1)+}+\mathrm{e}^{-+}+\mathrm{e}^{-}$, where $\mathrm{A}^{j+}$ represents the jth ionization stage of atom $\mathrm{A}$.

Since accurate quantum-mechanical calculation of this reaction is complex, recourse must be made to semi-empirical formulas. These rely upon the collision of the incident electron with the target ion approximating to a classical binary encounter between the incident and initially bound electrons. For example, there are Seaton's semi-empirical formula, (SEF), [32] and the exchange classical impact parameter, (ECIP), [33,34]. Typical results of applying Coulomb-Born calculations to some ionization cross-sections $[35,36]$ fall between ECIP and SEF and are convenient to use. The rate of collisional ionization for an electron can be written as [37] : 


$$
C_{j}^{\text {call }}=\left(1.09 \times 10^{-6} \mathrm{~cm}^{3} \mathrm{~S}^{-1}\right) n_{e l} n_{j} T^{1 / 2} e^{-\not \partial}\left(1-e^{-\not D}\right) \phi_{j}^{-2} \Gamma_{J}
$$

The various formulas in the literature differ primarily in their choice of the Gaunt factor, $\Gamma_{j}$. We use the empirical formula given in reference [37] because it combines all the various prescriptions to the Gaunt factor. In eq. (2-5) $n_{j}$ is the density of the ions in the jth state, $T$ is the electron temperature is $\mathrm{eV}$ and $\mathrm{x}_{\mathrm{j}} \equiv \Phi_{\mathrm{j}} / \mathrm{T}$ is the effective ionization potential in $\mathrm{eV}$, which is slightly reduced from the isolated atom ionization potential, $\Phi$, by the amount [38]

$$
\Delta \phi=e^{2} /\left(4 \pi \varepsilon_{0} \lambda_{D}\right),
$$

where $\lambda_{D}$ is the Debye shielding distance, $e$ is the elementary change, and $\varepsilon_{0}$ is the permittivity of free space.

The collisional recombination rate, which is the reverse process of collisional ionization, can be written as [37]

$$
\alpha_{j+1}^{c o l l}=C_{j}^{\text {coll }}\left(1.66 \times 10^{-22}\right) n_{\alpha L} T^{-3 / 2} e^{x_{j}}\left(u_{j+1} / u_{j}\right)
$$

where $C_{j}^{\text {coll }}$ is defined in equation $(2-5)$, and $u_{j}$ and $u_{j+1}$ are the electronic partition functions for the $\mathrm{jth}$ and the $(j+1)^{\text {st }}$ ionization stages.

For radiative recombination rate, we use the expression derived by Seaton [39].

$$
\alpha_{j+1}^{r a d}=\left(5.2 \times 10^{-14} \mathrm{~cm}^{3} S^{-1}\right) n_{a l} n_{j+1}(j+1) x_{j}^{3 / 2} e^{x_{j}} E_{1}\left(x_{j}\right)
$$

This is in accordance with the accuracy of assuming a simple coulomb field and using non-integral effective quantum numbers [40].

Dielectronic recombination becomes important at relatively high temperatures and low densities. For the dielectronic recombination rate we use [37] 


$$
\alpha_{j+1}^{d e l}=\left(2.4 \times 10^{-9} \mathrm{~cm}^{3} S^{-1}\right) n_{\alpha L} n_{j+1} T^{-32} B(j+1) D(j+1) \cdot \Sigma_{N} f_{n} A(y) e^{-E_{N}(S) T}
$$

where $i$ is the initial electronic state of the ion, and the summation is over all bound states $n, f_{n i}$ is the oscillator strength for the exciting transition. $B$ and $\bar{E}$ are given by

$$
\begin{aligned}
B(z \equiv j+1) & =z^{1 / 2}(z+1)^{5 / 2}\left(z^{2}+13.4\right)^{-1 / 2} \\
\bar{E}_{n j}(z) & =13.6 \mathrm{eV}(z+1)^{2}\left(v_{i}^{-2}-v_{n}^{-2}\right) / a
\end{aligned}
$$

where

$$
a=1+0.015(j+1)^{3} /(j+2)^{2}
$$

and $v_{i}$ and $v_{n}$ are the effective principal quantum numbers of state $i$ and $n$ respectively. The expressions for $A(y)$ and $D(j+1)$ are defined as:

$$
\begin{aligned}
& A(y)=y^{1 / 2} /\left(1+0.105 y+0.105 y^{2}\right) \\
& D(q=j+2)=N /\left(N_{t}+200\right)
\end{aligned}
$$

where

$$
y=(j+2) /\left(v_{1}^{-2}-v_{n}^{-2}\right)
$$

and

$$
N_{t}=\left[1.51 \times 10^{17}(j+1)^{6} T^{1 / 2} / n_{c}\right]^{1 / 7}
$$

\subsection{Criterion for Equilibrium}

As we shall see in chapter 3 , the flow field around the injected pellet is described by the fluid equations coupled with the rate equations (2-3). Hence, there are different 
time scales associated with the atomic processes and the fluid motion, such time scales are used to set the equilibrium and non-equilibrium flow limits.

It is of interest to examine the behavior of the rate equations (2-3), when the relaxation time, $\tau_{\mathrm{i}}$, of the atomic process is smaller or larger than the time required for a fluid element, $\tau_{\mathrm{f}}$, to encounter a significant change in temperature and density as it moves through the flow field. The assumption of equilibrium flow requires that $\tau_{i}$ be negligible compared with $\tau_{f}$ [24]. If $\tau_{i}$ is of the same order as $\tau_{f}$, then non-equilibrium effects must be taken into account [23]. Such effects can occur in connection with any of the molecular processes; translation, rotation, vibration, chemical composition, etc. These processes have been investigated by several authors in the study of problems such as non-equilibrium flow downstream of a strong shock wave, non-equilibrium nozzle flows, and chemically reacting flows $[23,24,41-45]$.

In addition to the above mentioned processes, non-equilibrium ionization has been investigated in the study of expanding flows and the flow field around hypersonic flight vehicles $[46,47]$.

The resulting theory, in general, showed that the equilibrium flow is the limiting case of non-equilibrium flow when the relaxation time of the rate processes tends to zero $[23,24]$. For a steady one-dimensional flow, the time derivative $d / d t$ for a fluid element becomes $v d / d r$, and so equation (2-3) can be written as,

$$
v d f_{j} / d r=n_{\text {ed }}\left[f_{j-1} C_{j-1}^{\text {coll }}-f_{j}\left(C_{j}^{\text {coll }}+\alpha_{j}^{\mathrm{cos}}\right)+f_{j+1} \alpha_{j+1}^{\mathrm{cos}}\right]
$$

Following Bray [23], an estimate of the relaxation time can be obtained from the inverse of ainy of the rates on the right hand side of equation (2-9), i.e. 


$$
\tau_{i}=1 /\left(n_{a L} f_{j-1} C_{j-1}^{\infty}\right)
$$

This is the ionization time required to obtain a specific stage of ionization. The corresponding flow time, $\tau_{\mathrm{f}}$, considered in this study is the pellet radius, $\mathrm{r}_{\mathrm{p}}$, divided by the flow velocity, $v, \tau_{f} \equiv r_{p} / v$, and hence, the equilibrium flow is valid approximation if $\tau_{i} \ll$ $\tau_{\mathrm{f}}$. Equilibrium calculations are performed using the equations set (2-4). If this criterion is violated, then non-equilibrium flow calculations are performed using equation (2-3). Thus for equilibrium to exist one expects that $\tau_{i} \ll \tau_{f}$ or:

$$
1 /\left[n_{a L} f_{0} C_{0}\right] \ll r_{p} / v
$$

If we define an ionization length, $\xi_{\mathrm{i}}$ as the flow velocity times the ionization time, then Eq. (2-10) becomes:

$$
\xi_{i} \ll r_{p}
$$

or

$$
\xi / r_{p} \ll 1
$$

For this study, the above ratio is calculated for typical conditions and pellet radii for the TEXT, TFTR, and CIT devices. The results are summarized in Table 1 and reveal that the nonequilibrium model is required. 
Table 1

\begin{tabular}{|c|c|c|c|c|}
\hline Device & $\mathrm{r}_{\mathrm{p}}(\mathrm{cm})$ & $\mathrm{n}_{\mathrm{e}}\left(10^{14} \mathrm{~cm}^{3}\right)$ & $\mathrm{T}_{\mathrm{e}}(\mathrm{keV})$ & $\xi_{\mathrm{i}} / \mathrm{r}_{\mathrm{p}}$ \\
\hline \hline TEXT & 0.025 & 0.3 & 1 & 340. \\
\hline TFTR & 0.025 & 0.5 & 5 & 3. \\
\hline CIT & 0.05 & 1 & 20 & 0.004 \\
\hline
\end{tabular}

In summary the rate equations, based on the collisional radiative model (CR), will be used to calculate the charge-state distribution for equilibrium and nonequilibrium flow. The atomic processes most relevant to this study are the collisional ionization, collisional recombination, radiative recombination, and dielectronic recombination. The validity of the much simpler equilibrium solution will be determined. In the following Chapter the rate equations appended to the fluid equations to form a general model for the chargestate equilibrium and nonequilibrium calculations. This general model will be applied to hydrogen and carbon pellets in Chapter 4 and 5 respectively. 

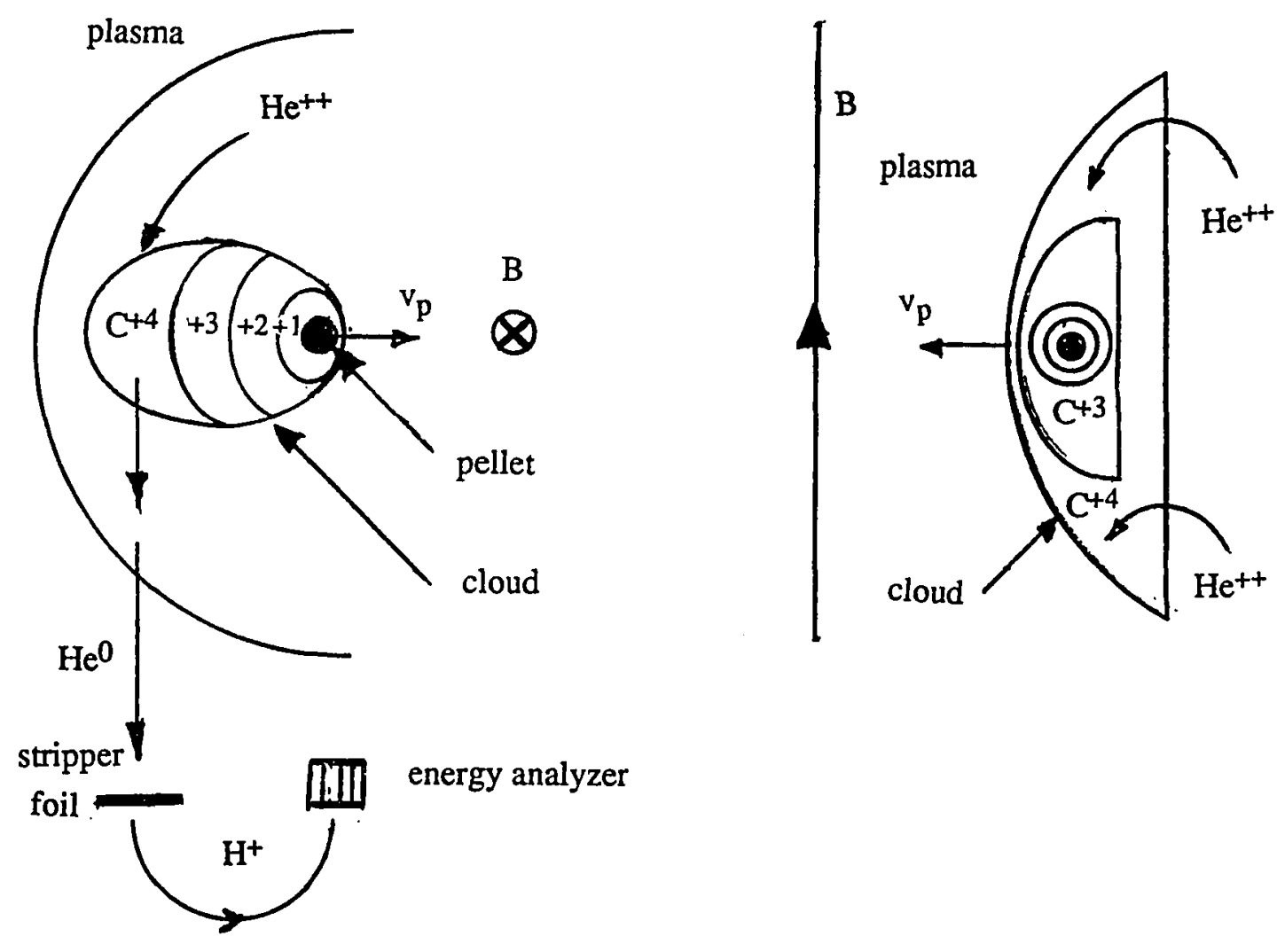

Fig. 2-1 Geometry of cloud. Alpha particles will interact with an ablation cloud surrounding an injected pellet. By observing helium neutrals, information on the alpha particles can be obtained. 


\section{Chapter 3.}

\section{THE GENERAL MODEL FORMULATION}

\subsection{Introduction}

The rate equations of chapter 2 are coupled to the gas-dynamic conservation equations in our development to the charge-state equilibrium and non-equilibrium models. In these models the heat transport to the pellet surface is accomplished via the external plasma particles. The energy lost by these particles is deposited within the cloud as a heat, and this links the plasma parameters to the gas density and remaining fluid and thermodynamic variables of the cloud through the constitutive gas-dynamic equations describing the ablatant. In this chapter in particular, we shall (1) give a discussion of the general assumptions used in this study, and (2) derive the governing equations for both equilibrium and non-equilibrium models.

\subsection{General Assumptions}

We describe the fluid equations for the ablation cloud surrounding the pellet, which include the atomic processes described in the previous chapter. The initial conditions used in this model are evaluated using the predictions of Felber et.al. [17] for hydrogen pellet and Parks et.al. [6] for carbon pellets. Hence, our assumptions follow closely these references. 
Thus the basic assumptions in this model as consistent with these references are:

1. Spherical expansion.

2. Steady-state flow.

3. Heat transport to the pellet is accomplished via plasma electrons.

4. Transonic flow.

5 Ionization energy will be treated in a self-consistent manner with the flow parameters in the energy balance.

6. Both nonequilibrium and equilibrium charge-state distribution will be studied.

We assume that the pellet is spherical and that the ablated material undergoes spherically symmetric expansion. This assumption has its known limitation, since it is observed experimentally that the spherical flow changes to channel flow after the cloud reaches a diameter of a few millimeters [21,48,49]. This assumption is discussed further in chapter 5 where the interaction of the ablatant cloud with the magnetic field, is studied.

The ablation is assumed steady. This is based on the fact that the characteristic time of the flow of the ablated material (time to establish the cloud; i.e. the pellet radius divided by the vapor flow velocity) is much shorter than the characteristic time of the recession of the pellet radius (pellet life-time), typical values for the times are $1 \mu$ s to establish the ablation cloud and $0.1 \mathrm{~ms}$ for the pellet life time.

Heat transport to the pellet surface is accomplished via plasma electrons that penetrate the ablatant. The effects of alphas and fast ions on the hydrogen-pellet cloud heating have been shown to be less important $[16,50]$ except in the outer regions of the plasma and hence these effects are not incorporated in this study. This assumption is 
reasonable because of the low relative speed of even the fast alphas with respect to the plasma electrons. Radiation transport within the ablation cloud is neglected. For the very low ablation termperature, the black body flux which is the worst case would be small compared with the incident electron heat flux.

The incident electron heat flux deposits its energy in the ablation cloud. This energy is used to heat, to ionize, and to accelerate the ablative flow. The ablatant leaves the pellet surface slowly because the electron heat flux at the pellet surface, $q_{0}$, is considered to be less than the heat flux from the background plasma, $\mathrm{q}_{\infty}$. The ablatant is then accelerated through heating and spherical divergence effects to supersonic velocity at less than one pellet radius from the surface [12]. Far from the pellet surface subsonic flow is restored. Therefore, transonic flow is assumed in this study.

The ablation process of the pellet is self-regulated by the surrounding cloud i.e., if the ablation rate on the pellet surface is enhanced this is accompanied by an increase in the cloud density which will decrease $\mathrm{q}_{0}$ through the shielding effect of the cloud. Therefore, the increase in the ablation rate is suppressed by the decrease in $\mathrm{q}_{0}$. On the other hand, if the ablation rate is reduced on the pellet surface, $q_{0}$ is increased to keep the stationary value of the cloud density. From this concept self-regulation, it is clear that the ablation rate of the pellet is strongly affected by the shielding mechanism of the cloud.

\subsection{Governing Equations}

The steady-state one-dimensional conservation laws for mass, momentum, and energy in the cloud, the rate equations introduced in Chapter 2, and the equation of state 
for the cloud, as an ideal gas with a constant ratio of specific heats, are written as follows:

$$
\begin{gathered}
\rho v r^{2}=\frac{G}{4 \pi}=\text { Constant } \\
\rho v \frac{d v}{d r}+\frac{d P}{d r}=0 \\
\frac{G}{4 \pi r^{2}} \frac{d}{d r}\left[\frac{\gamma T}{m(\gamma-1)}\left(1+\sum_{j=1}^{z} i f_{j}\right)+\frac{v^{2}}{2}+\frac{1}{m} \sum_{j=1}^{z} Q_{j} f_{j}\right]=\frac{d q}{d r} \\
v \frac{d f_{j}}{d r}=n_{a L}\left[f_{j-1} C_{j-1}^{c o l l}-f_{j}\left(C_{j}^{c o l l}+\alpha_{j}^{\cos }\right)+f_{j+1} \alpha_{j+1}^{\operatorname{lot}}\right] \\
P=n\left(1+\sum_{j=1}^{Z} i f_{j}\right)
\end{gathered}
$$

where $\rho, v, r, G, m, P$ represent the mass density, the fluid velocity, the radial distance from the center of the spherical pellet, the ablation rate, the mass of ablated atom, and the pressure of the cloud respectively. In equation (3-3), dq/dr means the heat source of the cloud due to plasma electrons, $T$ is the temperature of the cloud, $\gamma$ is the ratio of specific heats, and $f_{j}$ is the ionization fraction of the jth ionization state. The term,

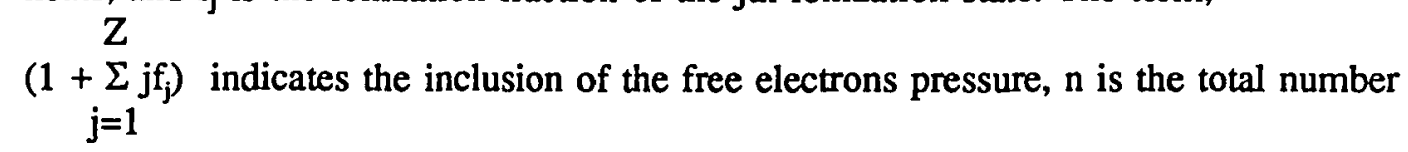
density of ablated atoms $(\rho / m)$, and $Z$ is the atomic number. The ionization energy term, $\Sigma Q_{j} f_{j}$, takes into account the energy required for an ion to achieve a multiple charge-state where. 


$$
Q_{j}=\sum_{i=1}^{j} \phi_{i}
$$

In order to close our model equations, we need an equation that describe the attenuation of the incident electron heat flux dq,/dr. As we shall see in Chapters 4 and 5 , the heat flux equation involves the vapor density. Therefore, this equation must be coupled to equations (3-1 to 3-5), and the whole set is then integrated simultaneously to obtain a self-consistent set of fluid profiles. These solutions, of course, depend on the boundary conditions. To avoid the expected singularity in the fluid equations (transonic flow) it is convenient to obtain solutions at the sonic surface denoted by $r=r_{*}$. This is the surface at which the flow velocity is equal to the local speed of sound, v.. Once these solutions are obtained, they are used as initial conditions to the fluid equations which can be propagated in the subsonic or supersonic regions of the cloud. In order to eliminate, $\mathrm{dp} / \mathrm{dr}$ from equation (3-2), we differentiate equations (3-1) and (3-5) to obtain:

$$
\frac{1}{\rho} \frac{d \rho}{d r}+\frac{2}{r}+\frac{1}{2 v^{2}} \frac{d v^{2}}{d r}=0
$$

and

$$
\frac{d P}{d r}=\frac{T L i}{m} \frac{d \rho}{d r}+\frac{\rho}{m} \frac{d}{d r}(T L i)
$$

where 


$$
L i \equiv 1+\sum_{j=1}^{z} i f_{j}
$$

Equation (3-2) can be rewritten as:

$$
\frac{d P}{d r}=-\frac{\rho}{2} \frac{d v^{2}}{d r}
$$

Using equation (3-7) and (3-8) gives:

$$
\frac{\rho}{2} \frac{d v^{2}}{d r}+\frac{T L i}{m} \frac{d \rho}{d r}+\frac{\rho}{m} \frac{d}{d r}(T L i)=0
$$

or

$$
\frac{1}{2} \frac{d v^{2}}{d r}+\frac{T L i}{m} \frac{1}{\rho} \frac{d \rho}{d r}+\frac{1}{m} \frac{d}{d r}(T L i)=0
$$

Finally, $\underline{1} \frac{\mathrm{d} \rho}{\mathrm{dr}}$ is replaced using equation (3-6) to obtain:

$\rho d r$

$$
\frac{d}{d r}(T L i)=T L i\left(\frac{2}{r}+\frac{1}{2 v^{2}} \frac{d v^{2}}{d r}\right)-\frac{m}{2} \frac{d v^{2}}{d r}
$$

Before proceeding any further, we normalize all quantities to their values on the sonic surface at $r=r_{*}$. Quantities evaluated at the sonic surface are denoted by a subscript asterisk. The normalized quantities are:

$$
\text { Z }
$$

where we have used $\mathrm{Li} \equiv 1+\Sigma \mathrm{jf}_{\mathrm{j}}$, and the local speed of sound $\mathrm{v}_{*}$ is given by: $\mathrm{j}=1$

$$
v_{*}=\left[\frac{\gamma T_{*} L_{*}}{m}\right]^{1 / 2}
$$




$$
\begin{aligned}
r^{\prime}=\frac{r}{r_{*}}, & \theta=\frac{T}{T_{*}} \\
n^{\prime}=\frac{n}{n_{*}}, & P^{\prime}=\frac{P}{P_{*}} \\
v^{\prime}=\frac{v}{v_{*}}, & W=v^{\prime} 2 \\
q^{\prime}=\frac{q}{q_{*}}, & L=\frac{L i}{L_{*}}
\end{aligned}
$$

Using the dimensionless quantities equations (3-3) and (3-10) becomes

$$
\begin{aligned}
& \frac{d}{d r^{\prime}}\left[\theta L+W\left(\frac{\gamma-1}{2}\right)\right]=S-A_{*} \frac{d}{d r^{\prime}} \sum Q_{j} f_{j} \\
& \frac{d}{d r^{\prime}}(\theta L)=\theta L\left(\frac{2}{r^{\prime}}+\frac{1}{2 W} \frac{d W}{d r^{\prime}}\right)-\frac{m}{2} \frac{d W}{d r^{\prime}}
\end{aligned}
$$

in which we have used

$$
\begin{gathered}
A_{*} \equiv \frac{(\gamma-1)}{\gamma T L_{*}} \\
S \equiv \frac{q_{*}}{n_{*} v_{*}} \frac{1}{n^{\prime} \sqrt{W}} \frac{d q^{\prime}}{d r^{\prime}} \\
G=4 \pi \rho_{*} v_{*} r_{*}^{2}
\end{gathered}
$$

Inserting equation (3-13) into equation (3-12) results in the governing equation for the change in dimensionless kinetic energy of the ablation cloud, $\mathrm{W}$, which is 


$$
\frac{d W}{d r^{\prime}}=\frac{2 W}{(\theta L-W)}\left[s-\frac{2 \theta L}{r^{\prime}}-A_{*} \frac{d}{d r^{\prime}} \sum_{j} Q_{j} f_{j}\right]
$$

The governing equation for the change in the dimensioniess temperature of the cloud, $\theta$, is obtain from equation (3-12), so

$$
\frac{d \theta}{d r^{\prime}}=\frac{1}{L}\left[S-\left(\frac{\gamma-1}{2}\right) \frac{d W}{d r^{\prime}}-A_{*} \frac{d}{d r^{\prime}} \sum_{j} Q_{j} f_{j}-\frac{\theta}{L_{*}} \sum_{j} j \frac{d}{d r^{\prime}} f_{j}\right]
$$

Finally, the normalized form of equation (3-4) is given by:

$$
\frac{d f_{j}}{d r^{\prime}}=\frac{n_{*} r_{*}}{v_{*} W r^{\prime 2}} \sum_{j} i f_{j}\left[f_{j-1} C_{j-1}^{c o l l}-f_{j}\left(C_{j}^{\text {coll }}+\alpha_{j}^{\cos }\right)+f_{j+1} \alpha_{j+1}^{\cos }\right]
$$

in which we have used

$$
n_{a b}=n \sum_{j} i f_{j}
$$

and

$$
n^{\prime} r^{\prime 2} \sqrt{W}=1
$$

Equations (3-14 to 3-16) as well as the heat flux equation, developed in the following chapters, are the general nonequilibrium model equations.

\subsection{The General Equilibrium Model}

Inspection of equation (2-4) shows that the degree of ionization, $\mathrm{f}_{\mathrm{j}}$, is a function of the ionization rate, and the collisional, radiative, and dielectronic recombination rates. These rates are functions of the local ablation cloud temperature and electron-density. 
Hence, the spatial derivatives of $f_{j}$, for the charge state equilibrium model, are transformed into expressions involving $\mathrm{dW} / \mathrm{dr}$ as shown in Appendix $\mathrm{A}$ and are given by

$$
\frac{d}{d r^{\prime}} \sum_{j=1}^{Z} Q f_{j}=U_{1}-U_{2} \frac{d W}{d r^{\prime}}
$$

and

$$
\sum_{j=1}^{Z} j \frac{d f_{j}}{d r^{\prime}}=U_{3}-U_{4} \frac{d W}{d r^{\prime}}
$$

where $U_{1}, U_{2}, U_{3}$, and $U_{4}$ are defined in Appendix $A$.

After elimination of the spatial derivatives of $f_{j}$, by using equations (3-17) and (3-18), equations (3-14) and (3-15) become,

$$
\begin{gathered}
\frac{d W}{d r^{\prime}}=\frac{2 W}{\left[\theta L-W\left(1+2 A_{*} U_{3}\right)\right]}\left[S-A_{*} U_{1}-\frac{2 \theta L}{r^{\prime}}\right] \\
\frac{d \theta}{d r^{\prime}}=\frac{1}{L}\left[S-A_{l} U_{1}-\frac{\theta U_{3}}{L_{*}}+\left[A_{*} U_{2}+\frac{\theta}{L_{*}} U_{4}-\left(\frac{\gamma-1}{2}\right)\right] \frac{d W}{d r^{\prime}}\right]
\end{gathered}
$$

Thus equations (2-4), (3-19) and (3-20) as well as the heat flux equation, dq/dr, are the charge-state equilibrium model equations. Note $\mathrm{dw} / \mathrm{dr}$ is no longer singular on the sonic surface [compare (3-19) and (3-14)].

\subsection{Method of solution}

The coupled, non-linear, first order ordinary differential equations describing the atomic processes and the ablation cloud flow described above will be solved numerically 
in the following chapters. An efficient, accurate algorithms for integrating these equations is required. One-step methods are the methods of choice because they require a minimal amount of information to be stored in the computer. Runge-Kutta methods are efficient, easily programmed one-step algorithms. The most commonly used Runge-Kutta method is the fourth-order explicit method.

Under conditions close to equilibrium, the differential term in the rate equations (3-16) is calculated as the small difference between large numbers and is very close to zero. For the integration to be stable, under these conditions, the integration step size must be very small and double precision calculation must be performed to retain sufficient accuracy. A small deviation from the correct solution produce a large change in the differential term in the rate equations, which tends to over-correct for the original error. Since most nonequilibrium flow problems involve departure from equilibrium initial conditions, this problem has received much attention $[51,52,53]$. One method of solution, due to Treanor [52] is to approximate the rate equation by a linear form over the range of one integration step. This linear form is then integrated explicitly, and the result is expressed in the form of the Runge-Kutta finite difference equation plus a correction term. The correction term allows relatively large steps in the near-equilibrium region. On the other hand, the correction term becomes small under conditions far from equilibrium, and then the method becomes identical with the fourth-order Runge-Kutta scheme.

In this study, the fourth-order Runge-Kutta scheme is used. To check the accuracy of the results, the integration step is varied and the resulting solution are compared. In some cases, Treaner technique [52] is used to test the accuracy of our results. The 
integration step used in this study is $0.001 r_{*}$, and all calculations were performed with double precision on the IBM 3090 mainframe computer at Old Dominion University. 


\section{Chapter 4.}

\section{APPLICATION TO HYDROGEN PELLETS}

\subsection{Introduction}

With $\mathrm{Z}=1$, the general model equations of the preceding chapter are simplified to obtain the model equations for hydrogen ablatant. The non-equilibrium flow is one in which departure from equilibrium occurs [23]; hence equilibrium initial conditions are considered.

With the necessary initial conditions in hand, the hydrogen ablation cloud flow with equilibrium and nonequilibrium ionization is studied. Specifically, the range over which the nonequilibrium criterion, introduced in chapter 2 , holds is determined.

\subsection{Hydrogen Model Equations}

For hydrogen ablatant, equations (3-14 to 3-16) which describe the nonequilibrium flow take the form:

$$
\begin{gathered}
\frac{d W}{d r^{\prime}}=\frac{2 W}{(\theta L-W)}\left[S-\frac{2 \theta L}{r^{\prime}}-A_{*} \phi_{*} \frac{d f_{1}}{d r^{\prime}}\right] \\
\frac{d \theta}{d r^{\prime}}=\frac{1}{L}\left[S-\left(\frac{\gamma-1}{2}\right) \frac{d W}{d r^{\prime}}-\left(A_{*} \phi_{1}+\frac{\theta}{L_{*}}\right) \frac{A_{1}}{d r^{\prime}}\right]
\end{gathered}
$$




$$
\frac{d f_{1}}{d r^{\prime}}=\frac{n_{e L_{*}} r_{*}}{v_{*} \sqrt{W}}\left[C_{o}^{z o t}-f_{1}\left(C_{o}^{c o t}+\alpha_{1}^{z o t}\right)\right]
$$

in which we have used

$$
\begin{gathered}
\alpha_{1}^{\text {cot }}=\alpha_{1}^{\text {coll }}+\alpha_{1}^{\text {rod }} \\
f_{0}+f_{1}=1 \\
C_{0}^{\text {cos }}=C_{0}^{x}+C_{0}^{\text {oll }} \\
C_{0}^{x}=\frac{n_{e}}{2 n_{e L}} S_{0}^{x}
\end{gathered}
$$

where $f_{0}, C^{\text {coll }}, S_{0}{ }^{x}$ are the fraction of ground-state hydrogen atoms, and ionization rate coefficients for the ablatant atoms by the ablatant electrons, and by external plasma electrons respectively. The factor of 2 , in the definition of $\mathrm{C}_{0}{ }^{\mathrm{x}}$, arises from the constraint that electrons enter only along the field lines.

In order to close the model equations, an equation that describe the attenuation of the incident electron heat flux, $q$, is required. As an approximation, the electron heat flux is modeled as a monoenergetic beam subject to a continuous slowing down processes $[12,17]$. This assumption is fairly restrictive and multigroup assumption has been successful in matching experimental results [22]. Multigroup electron distribution is included in the carbon ablatant treated in chapter 5 .

By assuming that the distribution function of the incident electrons is Maxwellian, the electron energy E, and heat flux q, far from the pellet asymptotically approach [12] 


$$
\begin{gathered}
E_{-}=2 T_{e} \\
q_{-}=\frac{n_{e} v_{e}}{4} E_{\infty}
\end{gathered}
$$

Where $n_{e}$ is the electron density, $T_{e}$ is the background plasma electron temperature, $v_{e}=(8 T / \pi m)^{1 / 2}$, and the product $\underset{4}{n_{e} v_{e}}$ is the particle flux.

The energy and heat flux degradation of the beam as it penetrates the ablatant is described by the stopping cross-section, or energy loss function $L(E)$, the elastic scattering cross-section $\sigma(E)$, and the neutral target density by the differential equations:

$$
\begin{gathered}
\frac{d E}{d r}=2 \frac{\rho}{m} L(E) \\
\frac{d q}{d r}=\frac{\rho}{m}\left[2 \frac{L(E)}{E}+\sigma(E)\right] q \\
\equiv \frac{\rho}{m} \wedge(E) q
\end{gathered}
$$

where $L(E)$ is given by the semiemprical expression in $H_{2}[17]$ as:

$$
L(E)\left(\mathrm{eVcm}^{2}\right)=86210^{-15} /\left[\left(\frac{E}{100}\right)^{.023}+\left(\frac{E}{60}\right)^{-1.25}+\left(\frac{E}{48}\right)^{-1.94}\right]
$$

$\Lambda(E) \equiv \sigma(E)+2 L(E) / E$ designate the effective cross-section for the electron heat flux attenuation, and $\sigma(E)$ for $\mathrm{H}_{2}$ is given by [17] 


$$
\sigma(E)\left(\mathrm{cm}^{2}\right)=8.810^{-13} E^{-1.71}-1.6210^{-12} E^{-1.932}
$$

The loss function $\mathrm{L}(\mathrm{E})$ and the electron scattering cross-section $\sigma(E)$ defined in equations (4-8) and (4-9) are taken to have in atomic hydrogen one-half of their values in molecular hydrogen.

Recall the normalized variables introduced in chapter 3, and further introduce the following variables:

$$
\begin{gathered}
\Lambda^{\prime}=\Lambda(E) / \Lambda\left(E_{*}\right) \\
E^{\prime}=E / E_{*} \\
L^{\prime}(E)=L(E) /\left[E_{*} \wedge\left(E_{*}\right)\right]
\end{gathered}
$$

Introducing the normalized variables in equations (4-6) and (4-7), and dropping the primes, the nonequilibrium model equations are:

$$
\begin{gathered}
\frac{d W}{d r}=\frac{2 W}{(\theta L-W)}\left[S-\frac{2 \theta L}{r}-A_{*} \phi_{1} \frac{d f_{1}}{d r}\right] \\
\frac{d \theta}{d r}=\frac{1}{L}\left[S-\left(\frac{\gamma-1}{2}\right) \frac{d W}{d r}-\left(A_{*} \phi_{1}+\frac{\theta}{L_{*}}\right) \frac{d f_{1}}{d r}\right] \\
\frac{d f}{d r}=\frac{n_{e L} r}{V_{*} \sqrt{W}}\left[C_{0}^{t o t}-f_{1}\left(C_{0}^{\infty 0 t}+\alpha_{1}^{\text {tot }}\right)\right] \\
\frac{d E}{d r}=2 \lambda_{*} \frac{L(E)}{r^{2} \sqrt{W}}
\end{gathered}
$$




$$
\frac{d q}{d r}=\lambda \cdot \frac{\Lambda q}{r^{2} \sqrt{W}}
$$

where the parameter $\lambda_{\text {a }}$ is given by the dimensionless quantity

$$
\lambda_{*}=\rho_{*} r_{*} \Lambda_{*} / m
$$

In a similar manner, equations (3-19), (3-20), (4-13), and (4-14) are brought together to form the charge-state equilibrium model equations for the hydrogen ablatant, viz.,

$$
\begin{gathered}
\frac{d W}{d r}=\frac{2 W}{\left[\theta L-W\left(1+2 A_{*} U_{2}\right)\right]}\left[S-A_{*} U_{1}-\frac{2 \theta L}{r}\right] \\
\frac{d \theta}{d r}=\frac{1}{L}\left\{S-U_{1}\left(A_{*}+\frac{\theta}{\phi_{1} L_{*}}\right)+\left[U_{2}\left(A_{*}+\frac{\theta}{\phi_{1} L_{*}}\right)-\left(\frac{\gamma-1}{2}\right)\right] \frac{d W}{d r}\right\} \\
\frac{d E}{d r}=2 \lambda_{*} \frac{L(E)}{r^{2} \sqrt{W}} \\
\frac{d q}{d r}=\lambda_{*} \frac{\Lambda q}{r^{2} \sqrt{W}}
\end{gathered}
$$

In this case the ionization fraction $f_{1}$ is given by Eq. (2-4), i.e.,

$$
f_{1}=\frac{R_{0,1}}{1+R_{0,1}}
$$

where

$$
R_{0,1}=\frac{C_{0}^{c o l l}+C_{0}^{x}}{\alpha_{1}^{c o l l}+\alpha_{1}^{\text {rad }}} \equiv \frac{C_{0}^{\text {tot }}}{\alpha_{1}^{\text {tot }}}
$$




\subsection{Initial Conditions}

Inspection of equation (4-1) shows that, the integral curve for the kinetic energy $\mathrm{W}$ passes through a singular point $\mathrm{r}=1$, since at this point $\theta=\mathrm{W}=\mathrm{L}=1$. For the equilibrium model, however, the singular surface exists just inside the sonic surface $(r=1)$ [17]. Therefore, initial conditions on the sonic surface must be evaluated first, and then the system equations are integrated to obtain solutions in the required direction.

If the atomic processes are neglected in the present model equations (4-10) - (414), one could obtain the neutral gas shielding (NGS) model equations [12]. In this NGS model, Parks and Turnbull solved a two-parameter eigenvalue problem to obtain the singular surface quantities. They defined the pellet surface boundary conditions, at the normalized pellet radius $\hat{r}_{p}\left(r_{p} / r_{*}\right)$, as

$$
q(\tilde{r})=0, \quad \theta(\tilde{r})=0
$$

This is a reasonable assumption, since the sublimation energy for hydrogen is vanishingly small compared with the electron heat flux at the sonic surface q. [12].

Initially $\lambda_{*}$ is chosen, then the derivative $d W / d r$ is evaluated by applying $L^{\prime}$ Hopital's rule. With all the derivatives known at the singular surface, they integrated their model equations inward $(r<1)$ until a point $r$ is reached such that the pellet surface boundary conditions (4-22) are satisfied. This locates the pellet surface. If no such point exists, then a different value of $\lambda_{*}$ is chosen. Knowing the correct value of $\lambda_{*}$, integration is performed outward ( $>1)$ until $q$ and $E$ approach their asymptotic value. The solutions correspond to a specific external plasma temperature. These solutions were plotted and a simple functional relationships for the quantities at the sonic surface, interms of $E_{\infty}$, 
were obtained. Such relationships can be used to obtain the sonic surface initial conditions, for a given background plasma temperature, without iteration.

The NGS model has been revised to include atomic processes [17]. In this model molecular dissociation of hydrogen ablatant was assumed complete at the pellet surface. Thus, the boundary condition requires every $\mathrm{H}_{2}$ molecule to absorb its dissociation energy of $4.5 \mathrm{eV}$ at the pellet surface. Hence, the energy flux deposited at the pellet surface is

$$
\Delta q=\frac{G}{2 m} \frac{4.5 e V}{4 \pi r_{p}^{2}}
$$

where $m$ is the mass of the hydrogen atom and $G$ is the ablation rate.

The new boundary conditions are that the heat flux be equal to $\Delta \mathrm{q}$ and that the temperature vanish at the pellet surface. The ionization state on the sonic surface was assumed to be in equilibrium with the flow, and hence the Saha equation was used to determine the fraction of hydrogen ions.

Applying the same procedure as in the NGS model, the sonic surface quantities are guessed initially until the pellet surface boundary conditions are satisfied. Introduction of the atomic processes increases the number of iteration considerably and makes it difficult to obtain a functional relationship for the sonic parameters because the governing equations depend on the choice of other variables as well as the external plasma temperature.

In order to extend the NGS model solutions to treat the atomic processes, Parks [19] assumed that the ablation density profile would not change by the inclusion of the atomic processes because it is controlled by the self-regulating nature of the ablation 
process. Moreover, he assumed that the sonic radius would not significantly altered by the atomic processes. Hence, the sonic surface temperature is the only variable which varies to balance for the electron heat flux consumed in the atomic processes. Gerdin [18] showed that these assumptions are consistent with the detailed numerical treatment of Felber et.al. [17]. Therefore, this procedure is used in the present study to obtain the initial conditions on the sonic surface.

In summary for hydrogen ablatant, the nonequilibrium model equations (4-10) to (4-14), and the charge-state equilibrium model equations (4-16) to (4-21) are based on the following basic assumptions

(1) One-dimensional steady-state transonic flow.

(2) Spherical pellet and symmetry.

(3) The incident electron heat flux is modeled by a monoenergetic beam.

(4) Ionization is treated self-consistently in the ablation cloud energy balance.

(5) Initial conditions on the sonic surface are evaluated using the models of references. $[12,19]$.

(6) The rate equation is used to calculate the fraction of ionization.

\subsection{Discussion of Results}

Recall the criterion for charge-state equilibrium, introduced in chapter 2 , which is written for hydrogen ablatant as 


$$
\frac{1}{n_{\alpha L} f_{1} C_{0}^{c o l l}} \ll \frac{r_{p}}{v}
$$

or

$$
\xi_{1} \ll r_{p}
$$

where $\xi_{i}=\frac{v}{n_{e L} f_{1} C^{\text {coll }}}$ is the ionization length.

In order to explore a range for the ratio $\xi_{j} / r_{p}$, tables $4-1$ to $4-3$ are constructed. Table 4-1 is calculated for a background plasma density of $1010^{14} / \mathrm{cm}^{3}$. The entry to any column gives the $\xi_{j} / r_{p}$ ratio at the sonic surface for a specific pellet radius and background electron temperature. Similarly, tables 4-1 and 4-3 are calculated for a density of $2 \cdot 10^{14}$ $\mathrm{cm}^{-3}$ and $3 \times 10^{14} \mathrm{~cm}^{-3}$ respectively. Inspection of these tables shows that for a high background plasma density and temperature and large pellet radius, the ratio $\xi_{j} / r_{p}$ tends to zero. For a small pellet and low background temperature this ratio is very large.

Tables 4-4 to 4-6 are obtained from tables 4-1 to 4-3 for a different values of the ratio $\xi_{\mathrm{i}} / \mathrm{r}_{\mathrm{p}}$. For each ratio, the minimum background electron temperature $(\mathrm{keV})$ is determined for any given combination of background plasma density and pellet radius.

The usefulness of the equilibrium criterion is then shown by performing both the equilibrium and nonequilibrium calculations when this criterion holds and when its violated. In Fig. 4-1 the hydrogen ablation cloud profiles Mach number M, total number density of the fluid $n$, flow velocity $v$, fractional ionization $f_{i}$, and ablation cloud temperature $\mathrm{T}$ for a case of ablation with charge-state equilibrium are compared with the 
corresponding profiles for a case of ablation with nonequilibrium ionization. For both cases the pellet radius is $0.28 \mathrm{~cm}$, the background plasma density and temperature are $1.0110^{14} \mathrm{~cm}^{-3}$ and $11.5 \mathrm{keV}$, and the ratio of the specific heats is $5 / 3$. These plasma conditions and pellet radius are chosen because the equilibrium solutions to this case are given by Felber et.al. [17] and completely agree with the solutions reported here. The ratio $\xi_{i} / r_{p}$ for this case is 0.28 .

Thus the limit $\xi_{j} / r_{p} \rightarrow 0$ leads to the equilibrium flow. This is in line with the intutive idea that an infinitely fast rate process will lead to the instantaneous establishment of local equilibrium as a fluid element moves through the flow.

In Fig. 4-2, where the equilibrium criterion is violated, the pellet radius is $0.2 \mathrm{~cm}$ and the background plasma density and temperature are $1.10^{14} \mathrm{~cm}^{-3}$ and $5 \mathrm{keV}$. For this case $\xi_{i} / r_{p}=3.7$ at the sonic surface and hence the assumption of equilibrium flow is not a working approximation.

In Fig (4-3), the predictions of both the equilibrium and nonequilibrium models are compared for $\xi_{j} / r_{p}=1$. The corresponding plasma conditions and pellet radius, obtained from table $4-1$, are $n_{e}=1.10^{14} \mathrm{~cm}^{-3}, T_{e}=6.33 \mathrm{keV}$ and $r_{p}=0.25 \mathrm{~cm}$. The agreement between both models is good for the charge-state distribution. Therefore, in nonequilibrium flow, the much simpler equilibrium solution will be sufficiently accurate for a given condition with $\xi_{i} / r_{p} \leq 1$. 


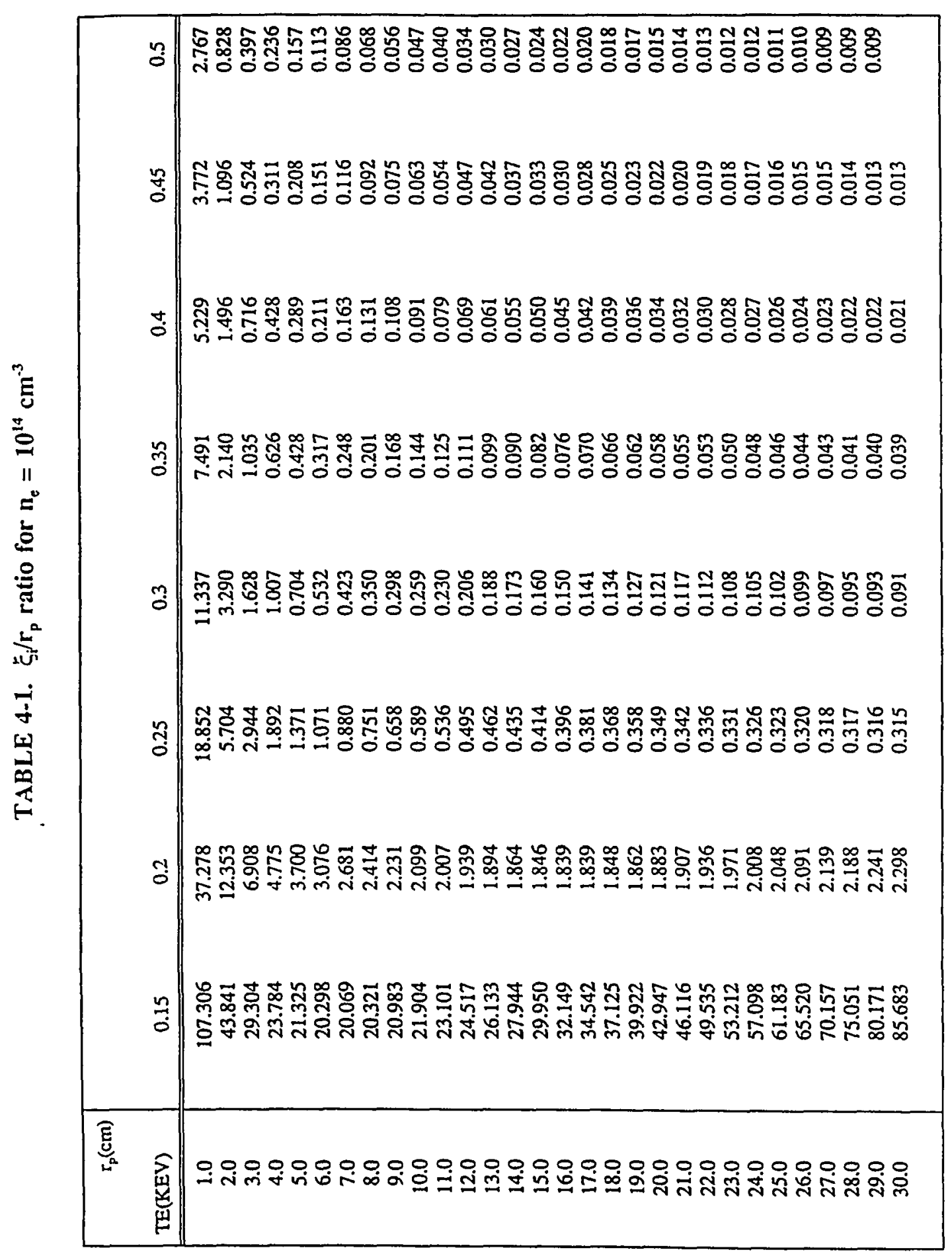




\begin{tabular}{|c|c|c|}
\hline Wّ: & 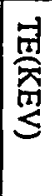 & 蒿 \\
\hline 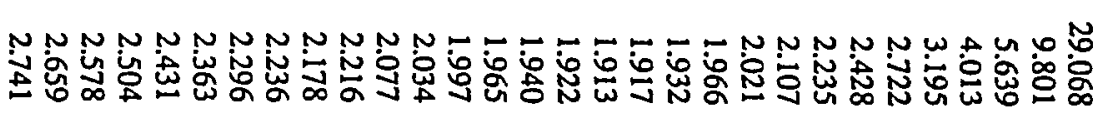 & 0 & \\
\hline 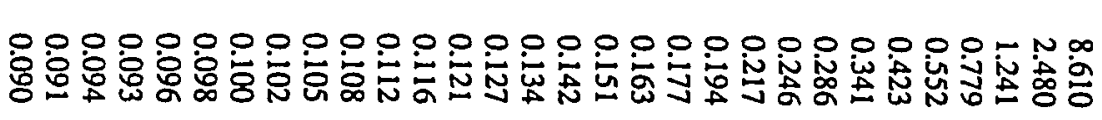 & 旅 & \\
\hline 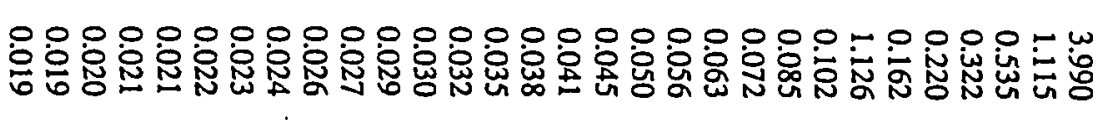 & is & \\
\hline 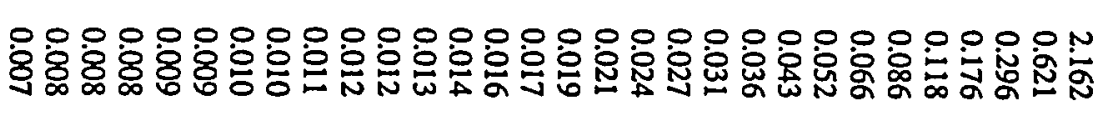 & 总 & \\
\hline 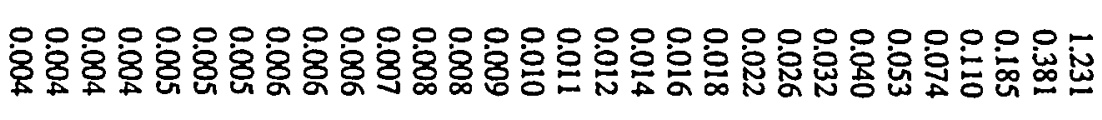 & is & \\
\hline 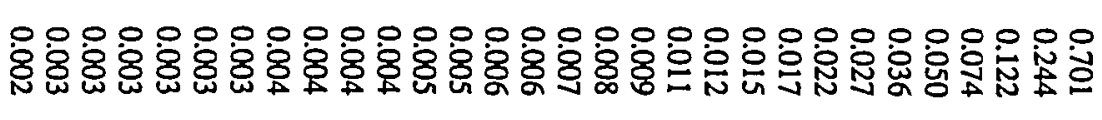 & $i_{u}$ & \\
\hline 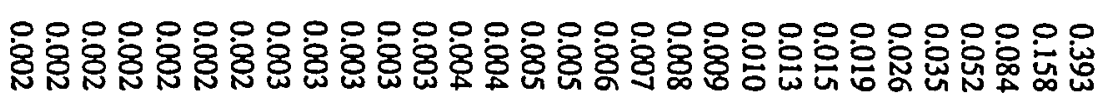 & $:$ & \\
\hline 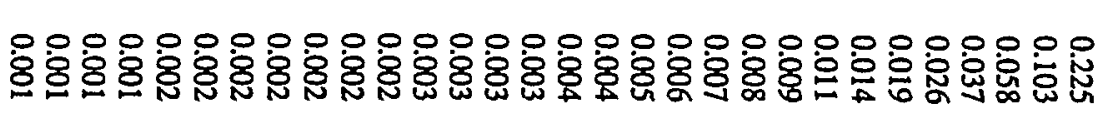 & iे & \\
\hline 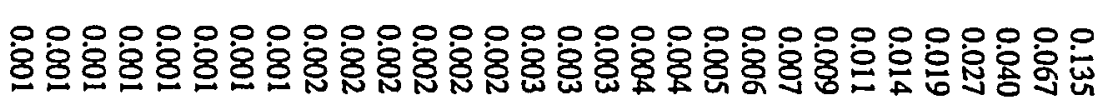 & in & \\
\hline
\end{tabular}




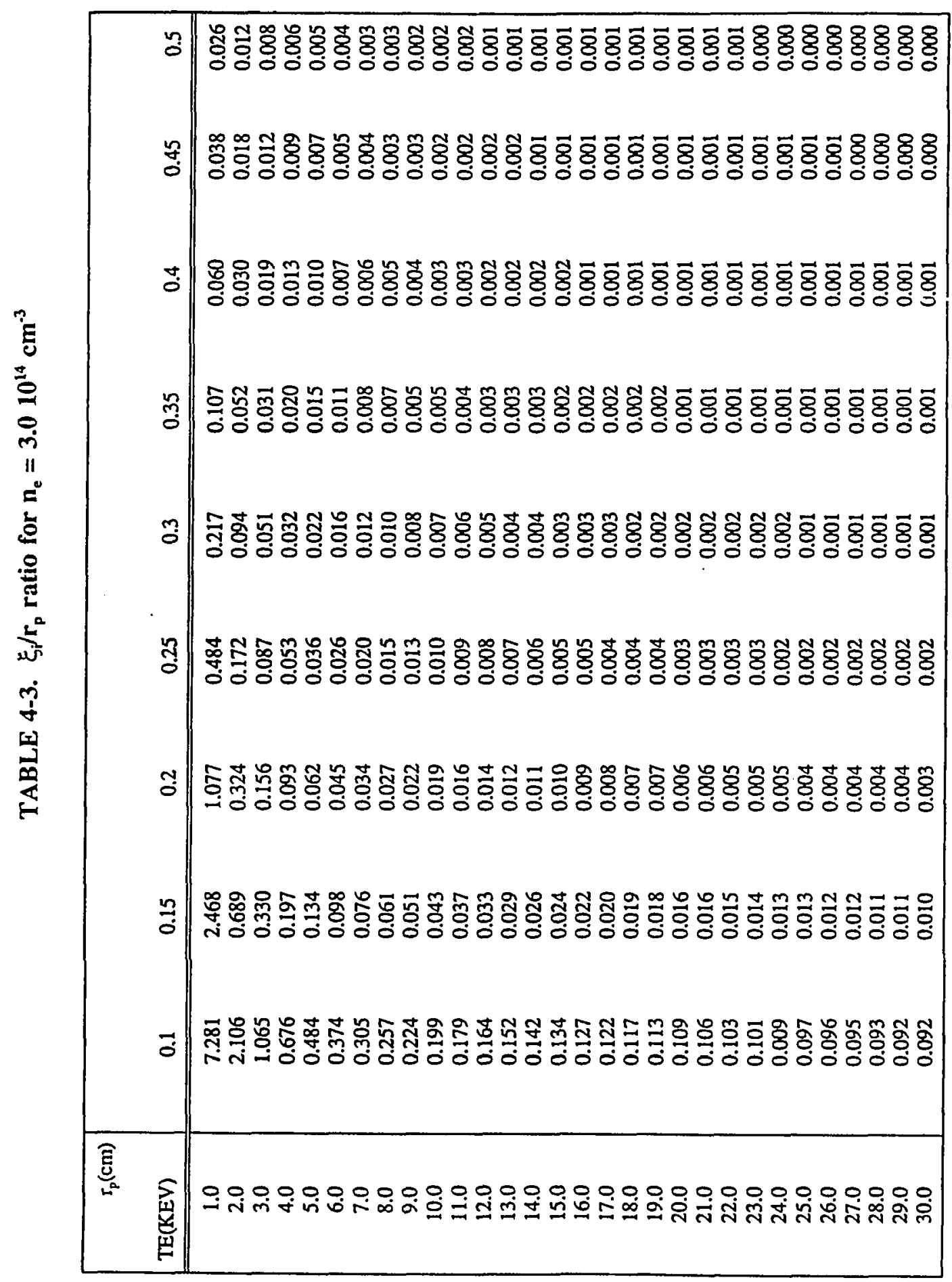


TABLE 4-4. The minimum background electron temperature $T_{e}$ for $\xi_{i} / r_{p} \leq 1.0$

\begin{tabular}{|c|c|c|c|}
\hline $\begin{array}{l}\mathrm{n}_{\mathrm{e}}\left(\mathrm{cm}^{-3}\right) \\
\mathrm{r}_{\mathrm{p}}(\mathrm{cm})\end{array}$ & $1.010^{14}$ & $2.0 \quad 10^{14}$ & $3.0 \quad 10^{14}$ \\
\hline 0.10 & * & * & 3 \\
\hline 0.15 & $*$ & 3 & 1 \\
\hline 0.20 & $*$ & 2 & 1 \\
\hline 0.25 & 6 & 1 & $* *$ \\
\hline 0.30 & 4 & 1 & $* *$ \\
\hline 0.35 & 3 & 1 & $* *$ \\
\hline 0.40 & 2 & 1 & $* *$ \\
\hline 0.45 & 2 & 1 & $* *$ \\
\hline 0.50 & 1 & 1 & ** \\
\hline
\end{tabular}

$* \mathrm{~T}_{\mathrm{e}}$ is unknown (>30 keV)

${ }^{* *} \mathrm{~T}_{\mathrm{e}}$ is less than $1 \mathrm{keV}$ 
TABLE 4-5. The minimum background electron temperature $T_{e}$ for $\xi_{i} / r_{p} \leq 0.1$

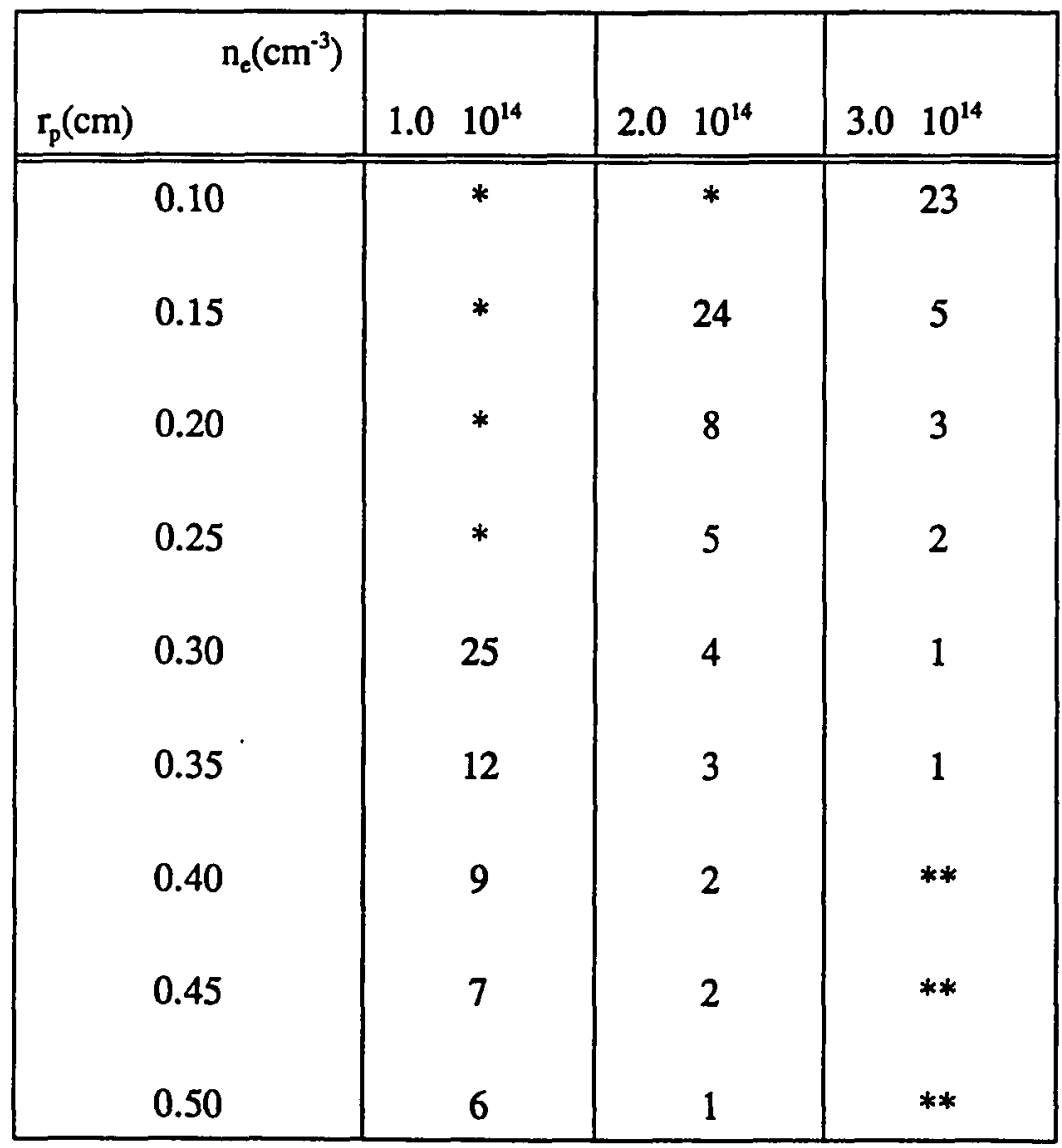

${ }^{*} \mathrm{~T}_{\mathrm{e}}$ is unknown (>30 keV)

${ }^{* *} \mathrm{~T}_{\mathrm{c}}$ is less than $1 \mathrm{keV}$ 
TABLE 4-6. The minimum background electron temperature $T_{e}$ for $\xi_{j} / r_{p} \leq 0.01$

\begin{tabular}{|c|c|c|c|}
\hline $\mathrm{r}_{\mathrm{p}}(\mathrm{cm})$ & $1.010^{14}$ & $2.010^{14}$ & $3.010^{14}$ \\
\hline 0.10 & * & * & * \\
\hline 0.15 & * & * & 30 \\
\hline 0.20 & * & * & 15 \\
\hline 0.25 & $*$ & 24 & 10 \\
\hline 0.30 & * & 16 & 8 \\
\hline 0.35 & * & 12 & 6 \\
\hline 0.40 & * & 10 & 5 \\
\hline 0.45 & * & 8 & 3 \\
\hline 0.50 & * & 7 & 2 \\
\hline
\end{tabular}

${ }^{*} \mathrm{~T}_{\mathrm{e}}$ is unknown ( $>30 \mathrm{keV}$ )

** $\mathrm{T}_{\mathrm{e}}$ is less than $1 \mathrm{keV}$ 


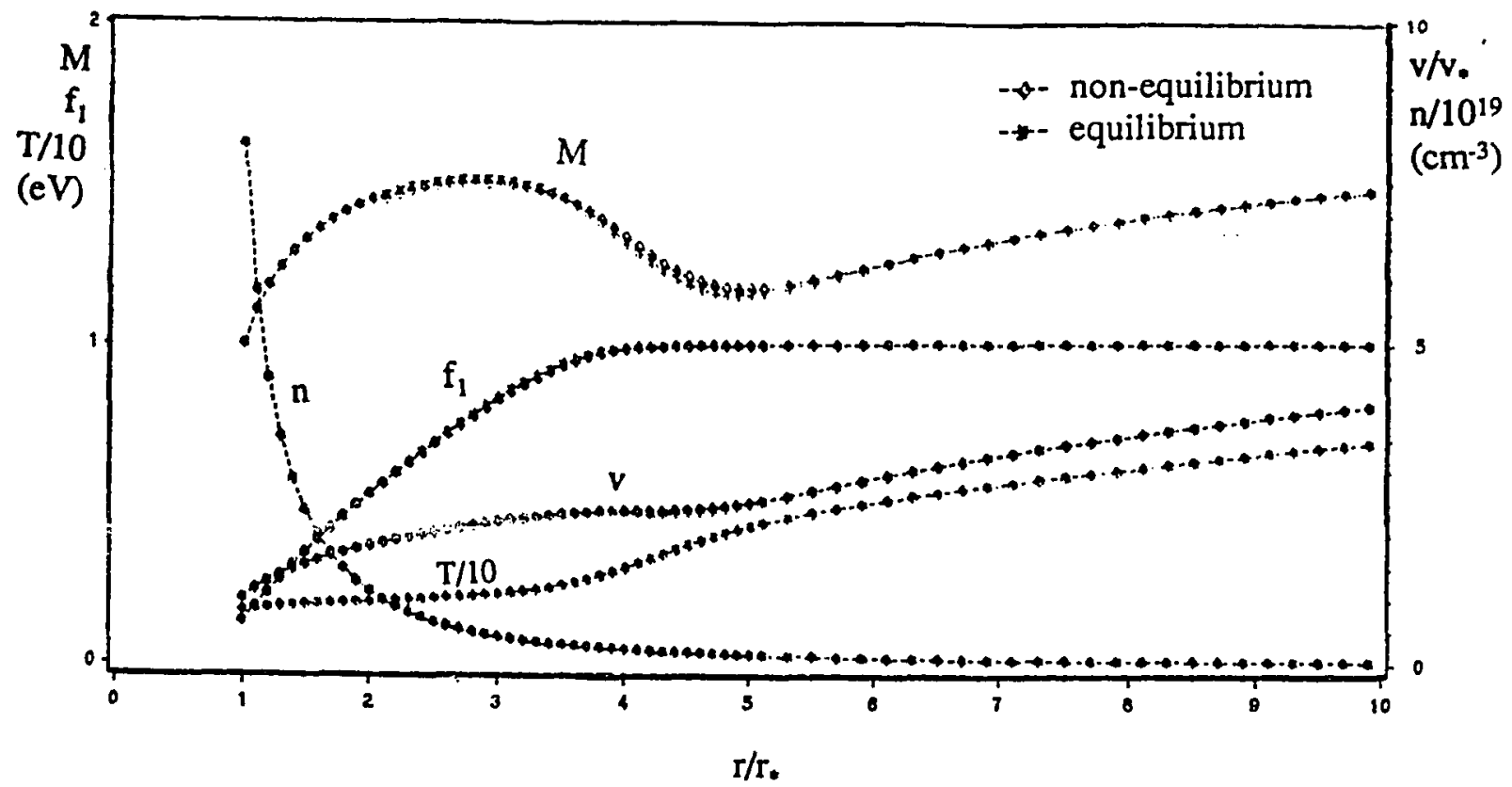

Fig. 4-1 Comparison of Mach number $M$, flow velocity v, total cloud number density n, cloud temperature $T$, and ionization $f_{1}$ for equilibrium and non-equilibrium hydrogen models. The pellet radius is $0.28 \mathrm{~cm}$, and the background plasma temperature and density are $11.5 \mathrm{keV}$ and $1.01 \times 10^{14} \mathrm{~cm}^{-3}$, respectively. $r_{*}=0.484 \mathrm{~cm}$ and $v_{*}=1.72 \times 10^{6}$ $\mathrm{cm} / \mathrm{s}$. 


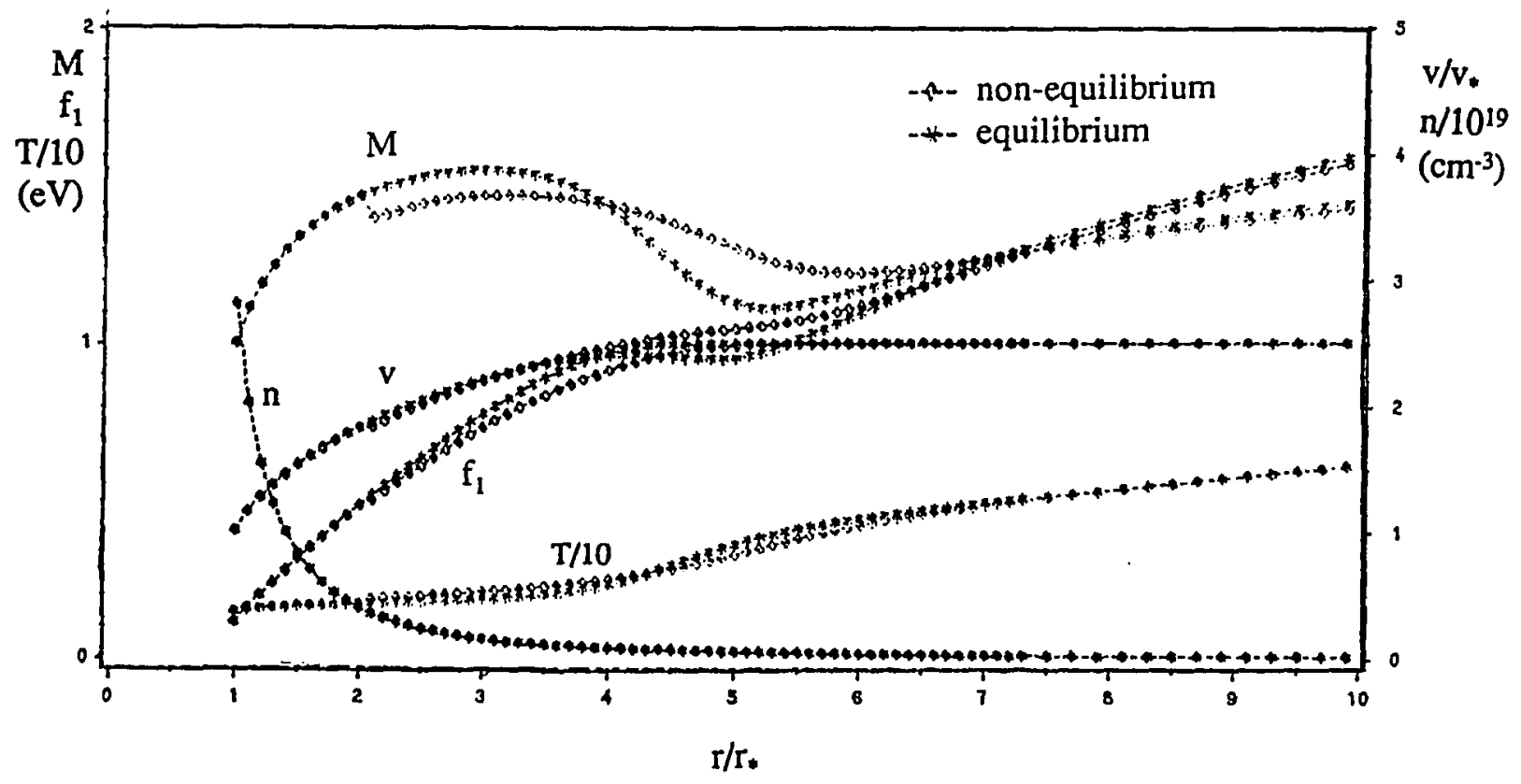

Fig. 4-2 Comparison of Mach number $M$, flow velocity $v$, total cloud number density $n$, cloud temperature $T$, and ionization $f_{1}$ for equilibrium and non-equilibrium hydrogen models. The pellet radius is $0.2 \mathrm{~cm}$, and the background plasma temperature and density are $5 \mathrm{keV}$ and $1.0 \times 10^{14} \mathrm{~cm}^{-3}$, respectively. $r_{*}=0.34 \mathrm{~cm}$ and $v_{*}=1.6 \times 10^{6} \mathrm{~cm} / \mathrm{s}$. 


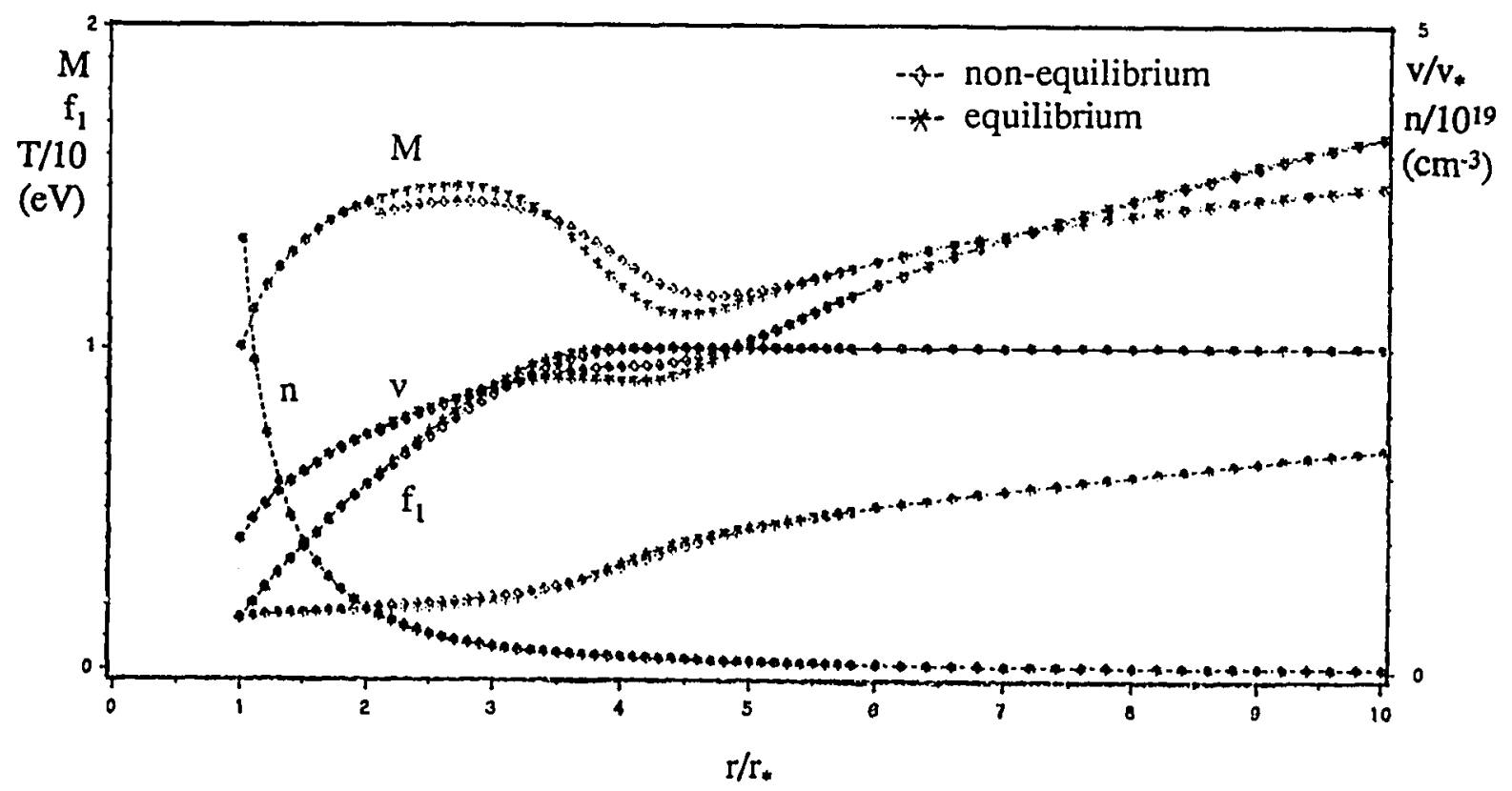

Fig. 4-3 Comparison of Mach number $M$, flow velocity $v$, total cloud number density $n$, cloud temperature $T$, and ionization $f_{1}$ for equilibrium and non-equilibrium hydrogen models. The pellet radius is $0.25 \mathrm{~cm}$, and the background plasma temperature and density are $6.33 \mathrm{keV}$ and $1.0 \times 10^{14} \mathrm{~cm}^{-3}$, respectively. $\mathrm{r}_{*}=0.427 \mathrm{~cm}$ and $\mathrm{v}_{*}=1.7 \times 10^{6}$ $\mathrm{cm} / \mathrm{s}$. 


\section{Chapter 5.}

\section{APPLICATION TO CARBON PELLETS}

\subsection{Introduction}

In the previous chapter hydrogen pellet was studied for two major reasons. The first is to gain confidence in our methodology and to feel comfortable with the model; since the physics of the hydrogen system is relatively easy to understand. Secondly, it is felt that determining a criterion for applying the equilibrium analysis is important to researchers interested in using hydrogen pellet for diagnostic purposes.

In this chapter, the nonequilibrium and equilibrium model equations, formulated in Chapter 3, are written for carbon ablatant. To close the model equations, an equation that describes the attenuation of the incident electron heat flux, $q$, is formulated where heating and slowing down of the plasma electrons are treated self-consistently. Also, the nonequilibrium and equilibrium models are upgraded by inclusion of the ionization by plasma electrons and the interaction of the ablation cloud with the magnetic field. Flow along the magnetic field lines is treated by placing a constraint in the flow area. This area constraint causes the flow to become singular in the supersonic region; this is interpreted as a shock wave. Since we are concerned with the net changes in the fluid properties across the shock and have no interest in studying the complex phenomena in the shock wave interior, a simplified model will be used. 
The models which we shall consider in this chapter can be summarized as follows:

\section{i. The Basic Model}

This includes the general model equations, discussed in Chapter 3, and the heat flux equation which we develop in the following section. The assumptions made for this model are as follows.

(1) One-dimensional steady-state transonic flow.

(2) Spherical pellet and symmetry.

(3) The incident electron heat flux is modeled by a multigroup electron distribution.

(4) Ionization is treated self-consistently in the ablation cloud energy balance.

(5) Initial conditions on the sonic surface are evaluated using the models of references $[6,56]$.

(6) Both equilibrium and/or nonequilibrium charge-state calculation are performed.

\section{ii. Plasma Electrons Model}

In this model, the ionization by the external plasma electrons is added to the features of the basic model.

\section{iii. Channel Flow Model}

This model includes all the features of the basic model but with a new expression for the area that takes into account the gradual conversion of the ablatant flow pattern from radial flow near the pellet surface to channel flow along the magnetic field lines. 


\section{iv. Complete Model}

This model consists of the channel flow model with the external plasma electrons effect included.

\subsection{Heat Flux Equation}

The electron heat flux is modeled as a multigroup electron distribution which is consistent with the low-Z pellet model developed by parks et.al. [6]. In this model the distribution function for electrons at the pellet surface is obtained by solving Boltzmann equation for plasma electrons slowing down in the cloud according to the Bethe-Block equation. This distribution is used to obtain an expression for the heat flux at the pellet surface in terms of the ablation cloud thickness for a given plasma conditions. The derivations for the heat flux degradation in carbon ablatant, $\mathrm{dq} / \mathrm{dr}$, are given in Appendix B.

\subsection{Carbon Pellet Models}

\section{3-1 The Basic Model}

Writing equations (3-14 to 3-16) and (B11) together forms the nonequilibrium basic model equations for carbon ablatant, i.e., 


$$
\begin{aligned}
& \frac{d W}{d r}=\frac{2 W}{(\theta L-W)}\left[S-\frac{2 \theta L}{r}-A_{*} \frac{d}{d r} \sum_{j=1}^{6} Q f_{j}\right] \\
& \left.\frac{d \theta}{d r}=\frac{1}{L}\left[S-\frac{(\gamma-1}{2}\right) \frac{d W}{d r}-A_{*} \frac{d}{d r} \sum_{j=1}^{6} Q f_{j}-\frac{\theta}{L_{*}} \sum_{j=1}^{6} \frac{d f_{j}}{d r}\right] \\
& \frac{d f_{j}}{d r}=\frac{n_{*} r_{*}}{v_{*} W r^{2}} \sum_{j=1}^{6} i f_{j}\left[f_{j-1} C_{j-1}^{\text {coll }}-f_{j}\left(C_{j}^{\text {coll }}+\alpha_{j}^{\cos }\right)+f_{j+1} \alpha_{j+1}^{\text {cot }}\right] \\
& \frac{d q}{d r}=C_{*} G(u) / \sqrt{W^{\prime}} r^{2}
\end{aligned}
$$

where $j=1,6$ and $C$. is defined as:

$$
C_{*}=\frac{9.7610^{-14} M n_{*} r_{*}}{T_{*}^{(p+1)}}
$$

Inspection of equation (5-1) shows that, the kinetic energy W passes through a singular point $r=1$, i.e. at the sonic surface where $\theta=W=L=1$. At this surface, the derivative $d W / d r$ is evaluated by applying L'Hopital's rule. Once $d w / d r$ is known, the other derivatives are evaluated at the sonic surface and the whole set is integrated outward.

The charge-state equilibrium basic model equations can be written from equations (3-19), (3-20), (B11), and (2-4), i.e.,

$$
\frac{d W}{d r}=\frac{2 W}{\left[\theta L-W\left(1+2 A_{*} U_{2}\right)\right]}\left[S-A_{*} U_{1}-2 \frac{\theta L}{r}\right]
$$




$$
\begin{gathered}
\frac{d \theta}{d r}=\frac{1}{L}\left[S-A_{*} U_{1}-\frac{\theta U_{3}}{L_{*}}+\left[A_{*} U_{2}+\frac{\theta U_{4}}{L}-\frac{(\gamma-1)}{2}\right] \frac{d W}{d r}\right] \\
\frac{d q}{d r}=C_{*} G(u) / \sqrt{W} r^{2} \\
f_{j}=\left(\prod_{m=0}^{j-1} R_{m, m+1}\right) /\left(1+\sum_{l-1}^{6} \prod_{m \rightarrow 0}^{l-1} R_{m, m+1}\right)
\end{gathered}
$$

where $j=1,6$ and $R_{m, m+1}$ is given by:

$$
R_{m, m+1}=\frac{C_{m}^{\text {coll }}}{\alpha_{m+1}^{\text {coll }}+\alpha_{m+1}^{\text {red }}+\alpha_{m+1}^{\text {del }}}
$$

Inspection of equation (5-5) shows that the singularity is removed from the kinetic energy equation at the sonic surface. This is due to the fact that the spatial derivatives of $f_{\mathrm{j}}$, in the kinetic energy equation, are transformed into expressions involving $d w / d r$. The singular surface for this model exists just inside the sonic surface [17], and hence the inward propagation would be very difficult.

\section{3-2 Plasma Electrons Model}

Up to this point we assumed ionization by ablatant electrons only. Plasma electrons could modify the charge-state profiles and the dimension of the cloud. Their effect will be more pronounced in the outer lower-density region of the cloud. The inclusion of these effects in our model is now considered. Convenient analytic expressions for the cross-sections or the non-Maxwellian rate coefficients for electron impact 
ionization are needed. Experimental and theoretical cross-section data for electron impact ionization of light atoms have been assessed by Bell et. al. [54]. Based on this assessment a recommended cross-section has been produced for each species investigated. For carbon species the recommended cross-section has been fitted by the following equation [54]

$$
\sigma(E)=\frac{1}{\phi E}\left\{A \ln (E / \phi)+\sum_{i=1}^{N} B_{i}\left(1-\frac{\phi}{E}\right)^{i}\right\}
$$

where $E$ is the incident electron energy, $\Phi$ is the ionization potential, and the coefficients $\mathrm{B}_{\mathrm{i}}$ are determined by a least squares fitting procedure. These coefficients are given in Appendix C. This formula ensures the correct behavior of the cross-section at both high and low impact energies.

The rate coefficients $<\sigma v>_{j}$ (cross-sections at a given energy multiplied by electron velocity $v_{e}$ at the same energy, evaluated over the distribution function for the plasma electrons slowing down in the ablatant cloud) are derived in Appendix C.

The rate equations, including ionization by external plasma electrons, can be written as

$$
\frac{d f_{j}}{d t}=n_{e L}\left\{f_{j-1}\left(C_{j-1}^{c o l l}+C_{j-1}^{x}\right)-f_{j}\left(C_{j}^{\text {coll }}+C_{j}^{x}+\alpha_{j}^{\text {tot }}\right)+f_{j+1} \alpha_{j+1}^{\text {tot }}\right\}
$$

in which we have used

$$
\begin{aligned}
C_{j}^{x} & =\frac{n_{e}}{n_{e L}} S_{j}^{x} \\
S_{j}^{x} & \equiv<\sigma . v\rangle_{j}
\end{aligned}
$$


where $n_{e}$ is the background electron density and $n_{e l}$ is the ablatant electron density.

Under steady state conditions the ionization fractions, $f_{j}$, are determined by equation (2-4) or (5-9) with $\mathrm{df}_{\mathrm{j}} / \mathrm{dt}=0$, viz,

$$
f_{j}=\left(\prod_{m=0}^{j-1} R_{m, m+1}\right) /\left(1+\sum_{l=1}^{Z} \prod_{m=0}^{l-1} R_{m, m+1}\right)
$$

where $R_{m / m+1}$ take new forms, namely

$$
R_{m, m+1} \equiv \frac{C_{m}^{\text {coll }}+C_{m}^{x}}{\alpha_{m+1}^{\text {coll }}+\alpha_{m+1}^{\text {rad }}+\alpha_{m+1}^{\text {del }}}
$$

The non-equilibrium model equation, with plasma electrons effects, are the same except the rate equations. Therefore the propagation equations in the non-equilibrium plasma electrons model are (5-1), (5-2), (5-4) and (5-9).

The spatial derivatives of $f_{j}$ are transformed into expressions involving $d W / d r$, see Appendix D, and are given by

$$
\begin{gathered}
\frac{d}{d r^{\prime}} \sum_{j=1}^{z} Q f_{j}=U_{1}^{x}-U_{2}^{x} \frac{d W}{d r^{\prime}} \\
\sum_{j=1}^{z} j \frac{d f_{j}}{d r^{\prime}}=U_{3}^{x}-U_{4}^{x} \frac{d W}{d r^{\prime}}
\end{gathered}
$$

where $U_{1}{ }^{x}, U_{2}{ }^{x}, U_{3}{ }^{x}$, and $U_{4}{ }^{x}$ include the external plasma electrons effect and are defined in Appendix D. In a similar manner equations (5-12) and (5-13) are inserted into equations (5-20) and (5-21) so, 


$$
\begin{gathered}
\frac{d W}{d r}=\frac{2 W}{\theta L-W\left(1+2 A_{*} U_{2}^{x}\right)}\left[S-A_{*} U_{1}^{x}-\frac{2 \theta L}{r}\right] \\
\frac{d \theta}{d r}=\frac{1}{L}\left\{S-A_{*} U_{1}^{x}-\frac{\theta U_{3}^{x}}{L_{*}}+\left[A_{*} U_{2}^{x}+\frac{\theta}{L_{*}} U_{4}^{x}-\left(\frac{\gamma-1}{2}\right)\right] \frac{d W}{d r}\right\}
\end{gathered}
$$

Finally, in the charge-state equilibrium plasma electrons model, the propagation equations are (5-7), (5-10), (5-14) and (5-15).

\section{3-3 Channel Flow Model}

Up to this point the model is still restricted by the assumption of spherically symmetric expansion of the ablation cloud, since it is observed experimentally that the spherical flow change to channel flow along the magnetic field lines [21, 48, 49]. Therefore, the model needs modifications to include the interaction of the ablation cloud with the magnetic field, so its predictions can be compared with experimental results.

The channelling of the flow along the magnetic field lines is treated phenomenologically by restricting the cross-sectional area of the flow $A(r)$ to be:

$$
A(r)=2 \pi r_{c h}^{2}\left[1-\exp \left(-\alpha r^{2}\right)\right]
$$

where $\mathrm{r}_{\mathrm{ch}}$ is the channel half-width and the parameter $\alpha=2 / \mathrm{r}_{\mathrm{ch}}{ }^{2}$. In this channel flow model, the density of the ablation cloud fall off much less rapidly after the ablatant is forced to flow along the magnetic field lines as opposed to that predicted by the spherical expansion. 
The conservation law for mass (mass rate of flow), equation (3-1), is now written in terms of $A(r)$, i.e.,

$$
\rho(r) v(r) A(r)=G=\text { constant }
$$

Equations (3-2) to (3-5) remain unchanged. In a completely analogous way, equations (32) to (3-5) and equation (5-17) are simplified to yield the counterpart of equations (3-14) to $(3-16)$, viz.,

$$
\begin{aligned}
& \frac{d W}{d r}=\frac{2 W}{(\theta L-W)}\left[S-\frac{\theta L}{A} \frac{d A}{d r}-A_{*} \sum Q_{j} \frac{d f_{j}}{d r}\right] \\
& \frac{d \theta}{d r}=\frac{1}{L}\left[S-\left(\frac{\gamma-1}{2}\right) \frac{d W}{d r}-A_{.} \sum Q_{j} \frac{d f_{j}}{d r}-\frac{\theta}{L_{*}} \sum_{j} j \frac{d f_{j}}{d r}\right] \\
& \frac{d f_{J}}{d r}=\frac{n_{*} r_{*}}{V_{*} W A} \sum j f_{j}\left[f_{j-1} C_{j-1}^{\text {coll }}-f_{j}\left(C_{j}^{\infty o l l}+\alpha_{j}^{\cot }\right)+f_{j+1} \alpha_{j+1}^{\infty 0 t}\right]
\end{aligned}
$$

In which we have used $n^{\prime} \sqrt{W} A^{\prime}=1$, and the primes on the dimensionless quantities in equations (5-18) to (5-20) are dropped.

Finally, the heat flux equation (5-4) becomes:

$$
\frac{d q}{d r}=C_{*} G(u) \mid A \sqrt{W}
$$

One should note that the channel flow nonequilibrium model equations (5-18 to $5-21$ ) could have been obtain directly from equations (5-1 to 5-4) by replacing $r^{2}$ by $A^{\prime}\left(r^{\prime}\right)$ and $\frac{2}{r^{\prime}}$ in $d W / d r$ equation by $\frac{1}{A^{\prime}} \frac{d A^{\prime}}{d r^{\prime}}$. This direct analogy is used to write the 
channel flow equilibrium model equations from equations (5-5 to 5-8), i.e.,

$$
\begin{gathered}
\frac{d W}{d r}=\frac{2 W}{\theta L-W\left(1+2 A_{*} U_{2}\right)}\left[S-A_{*} U_{1}^{\prime}-\frac{\theta L}{A} \frac{d A}{d r}\right] \\
\frac{d \theta}{d r}=\frac{1}{L}\left[S-A_{*} U_{1}^{\prime}-\frac{\theta U_{3}^{\prime}}{L_{*}}+A_{*} U_{2}^{\prime}+\frac{\theta U_{4}^{\prime}}{L}-\left(\frac{\gamma-1}{2}\right) \frac{d W}{d r}\right] \\
\frac{d q}{d r}=C_{*} G(u) / A \sqrt{W} \\
f_{J}=\left(\prod_{m=0}^{j-1} R_{m, m+1}\right) /\left(1+\sum_{l=1}^{6} \prod_{m=0}^{l=1} R_{m, m+1}\right)
\end{gathered}
$$

where $U_{1}^{\prime}, U_{2}^{\prime}, U_{3}^{\prime}$ and $U_{4}^{\prime}$ are obtained from equations (A37) to (A40) after writing $V_{j}$, equation (A31), as

$$
V_{J}=\frac{S A_{j}}{L}-\frac{B_{j}}{A} \frac{d A}{d r}
$$

Another expression for the area that takes into account the cloud diffusion across the magnetic field lines, is given by [55]:

$$
A_{M A G}=r_{c h}^{2} x\left[1-\exp \left(-\frac{\left(\xi / r_{c h}\right)^{2}}{x}\right)\right]
$$

where $r_{c h}$ is the channel half-width normalized to $r_{n}, \xi=r / r_{*}$ and the parameter $x$ is defined as 


$$
x=\left[1+\xi / r_{c h}\right]^{1 / 2}
$$

\section{3-4 The Complete Model}

This model combines the plasma electrons and the channel flow models in one. Therefore the nonequilibrium equations are the same as Eqs. (5-18) to (5-20) but the term $\mathrm{C}^{\mathrm{coll}}{ }_{\mathrm{j}-1}$ is replaced by $\mathrm{C}_{\mathrm{j}-1}^{\mathrm{do}}$ where:

$$
C_{J-1}^{10 x}=C_{J-1}^{\text {coll }}+C_{J-1}^{x}
$$

and $C_{j-1}^{x}$ is given by:

$$
C_{j}^{x}=\frac{n_{e}}{n_{e L}} S_{j}^{x}
$$

\subsection{Initial Conditions}

The initial conditions for carbon ablatant are determined according to the low- $\mathrm{Z}$ pellets model developed by Parks et.al. [6]. Their approach and assumptions are similar to the hydrogen pellet model $[12,17]$, but this model does not provide solution to the fluid equation. However, it establishes the sonic surface variables self-consistently. The model is based on the following major assumptions.

1. The ablatant density profile in the subsonic region falls as $r^{-\alpha}$, where $r$ is the radial distance from the center of the pellet and $5<\alpha<6$.

2. The ratio of the sonic radius to the pellet radius $r / r_{p}$ is $4 / 3$.

3. The Mach number of the ablatant at the pellet surface is 0.5 . 
4. The temperature of the ablatant at the pellet surface is $0.6 \mathrm{eV}$ in the highheat flux regime and $20 \%$ lower in the low-heat flux regimes.

5. The conditions of local thermodynamic equilibrium prevail, an hence the Saha equation is used to calculate the equilibrium ionization fraction.

Vahala et.al. [56] tested these assumptions by solving the steady flow problem by means of a two-parameter shooting code in a manner similar to those previously used for hydrogen pellets $[12,17]$ and discussed in chapter 4 . The theoretical predictions obtained from applying the suggested assumptions are compared with those predicted from the vigorous analysis [56] and proved satisfactory. The assumption that LTE prevails appears to be in reasonable agreement for high density TFTR/CIT regime, but it appears to break down for the low-density TEXT regime [56]. A better agreement with the TEXT and lowdensity TFTR experiments is reported when no ionization, in the inner region of the ablatant, is assumed in their calculations [56]. Therefore, the low- $Z$ pellet model [6] is used to determine the sonic-surface conditions for the high-density devices. For lowdensity devices, the low- $Z$ model is modified by assuming no ionization at the sonic surface. The assumptions of charge-state equilibrium at the sonic surface is used as an initial conditions for the equilibrium and nonequilibrium models for predictions involving TFTR and CIT.

\subsection{Shock Equations}

The results of the channel flow model presented in the next section show that the flow becomes singular in the supersonic region. This is interpreted as a shock in an analogous manner to supersonic flow in a nozzle [57]. The case of a stationary straight 
shock wave oriented perpendicular to the flow direction (i.e., a normal shock) is considered. Unless one is interested in studying the structure of the shock wave, it is usually possible to consider the shock wave to be infinitesimally thin (i.e., a mathematical discontinuity) and use the conservation laws of mass, momentum and energy for the ablatant fluid to determine the changes in flow properties across the shock wave [57]. The conditions upstream of the shock wave are designated with a subscript 1 while the conditions downstream are designated with a subscript 2 . For the nonequilibrium channel flow model the temperature, kinetic energy and density ratios are expressed as functions of the upstream Mach number, $M_{1}$, (see Appendix E), i.e.,

$$
\begin{gathered}
\frac{\theta_{2}}{\theta_{1}}=\frac{\left[2 \gamma M_{1}^{2}-(\gamma-1)\right]\left[2+(\gamma-1) M_{1}^{2}\right]}{(\gamma+1)^{2} M_{1}^{2}} \\
\frac{W_{2}}{W_{1}}=\left[\frac{2+(\gamma-1) M_{1}^{2}}{(\gamma+1) M_{1}^{2}}\right]^{2} \\
\frac{n_{2}}{n_{1}}=\frac{(\gamma+1) M_{1}^{2}}{2+(\gamma-1) M_{1}^{2}}
\end{gathered}
$$

In deriving the above equations the atomic processes are considered frozen across the discontinuity. For the equilibrium channel flow model, it is assumed that the ablatant is in instantaneous charge-state equilibrium on both sides of the discontinuity. The corresponding ratios in this case are obtained by performing an iterative numerical solution to the conservation equations for the ablatant fluid across the shock (see Appendix E). 


\section{$5.6 \quad$ Results}

The results of equilibrium and nonequilibrium basic models, presented in section 5.3, are shown in Figs. (5-1) to (5-3) for carbon pellets exposed to a variety of fusion plasma conditions. Equilibrium initial conditions are assumed for each case and the nonequilibrium solutions are switched at $r=2 r$.. The ionization length, discussed in Chapter 2, divided by the pellet radius $r_{p}$ (i.e. the ratio $\xi / r_{p}$ ) is calculated for the given conditions. The criterion for equilibrium, as given in chapter 4 , requires that the ratio $\xi_{i}$ $\leq 1$. In Figs. (5-1), the equilibrium and nonequilibrium calculations are shown for comparison, and the ratio $\xi_{/} / r_{p}$ for this case for the first charge state $f_{1}$ calculated at the sonic surface is 0.004 . Based on the equilibrium criterion mentioned above for hydrogen pellets, the equilibrium approximation is valid. For such a small ratio the ionization is almost instantaneous and there is good agreement between both solutions in the inner regions $\left(r<7 r_{*}\right)$ as shown in Fig. (5-1). As $r$ increases, the density falls off and hence the ratio $\xi_{/} / r_{\mathrm{p}}$ increases (see Eq. 4-24). The reason the $\xi_{/} / r_{\mathrm{p}}$ criterion does not hold for carbon for all $r>r_{*}$ as it did for hydrogen is that hydrogen has only one electron, which is stripped away much closer to the pellet surface (where equilibrium still holds) whereas the higher ionization states of carbon persist to much lower densities (where LTE and equilibrium breakdown) therefore one would expect that solutions will disagree as $\mathbf{r}$ increases. Indeed, at $r>7 \mathrm{r}$. the departure of equilibrium solution from the nonequilibrium is obvious in Fig. (5-1).

The key factor accounting for the departure of the nonequilibrium and equilibrium calculations is the ionization process which represents an important channel in the energy 
balance [see Eqn. (5-1)]. Therefore, if the fraction of the external electron heat going into ionization differs, the external electron heat going into thermal ablatant energy will also differ and consequently the rest of the flow variables, including the flow velocity $\mathrm{v}$ and the Mach number M, will diverge from one another. In the same Fig. (5-1), the charge state distribution $f_{1}$ is typically the same for both equilibrium and nonequilibrium, and therefore the rest of the flow parameters are almost identical in the region $r . \leq r \leq 7$. For $r>7 r$ one sees that the predictions of the nonequilibrium calculations, for the higher charge-state distribution $\left(f_{2}, f_{3}, f_{4}\right)$, lags those predicted form the equilibrium model. This behavior is expected because this delay represents the time required for the ionization process to take place. This difference is felt in the energy balance and manifests itself in the ablation cloud temperature. As we see in the same Fig. (5-1), during the ionization the temperature is almost flat, for both calculations. When the ionization is nearly complete, the incident electron heat flux looses an energy sink and heats up the ablation cloud, raising its temperature which causes a dip in the Mach number.

In Fig. (5-2), the charge-state distributions and the flow variables for both equilibrium and nonequilibrium are shown for comparison. For this case the ratio $\xi_{j} / r_{p}$ at the sonic surface for the first charge state is 0.02 and therefore both calculations still show a reasonable agreement in the inner regions, $r<5 r_{*}$. For $r>5 r_{*}$, the equilibrium solutions begin departing from the nonequilibrium solutions.

In Fig. (5-3), the equilibrium and nonequilibrium predictions are shown for comparison. The ratio $\xi_{j} / r_{p}$ for the first charge state at the sonic surface is 7.6 . For this large ratio, the flow is too fast to ionize instantaneously and the departure of equilibrium 
solutions from nonequilibrium ones is quite obvious right at the switching point $\left(r=2 r_{*}\right)$. This means that the assumption of equilibrium is not a useful working approximation even at the sonic surface, since the equilibrium criterion is violated. For such cases where $\xi_{j} / r_{p}>1$ at the sonic surface, the equilibrium initial conditions should not be used and the zero ionization initial conditions are most appropriate [56]. In the rest of this study, only nonequilibrium calculations will be presented. The initial conditions are selected according to the ratio $\xi_{i} / r_{p}$ for the first charge-state at the sonic surface. Equilibrium initial conditions are assumed if this ratio is less than one.

In order to show the difference between the various models presented in this chapter and the effects of the upgrades to the basic model, Figures (5-4) to (5-7) are calculated for a typical CIT condition. Fig. (5-4) shows the predictions of the basic nonequilibrium model. The predictions of the plasma electrons model shown in Fig. (5-5) are compared with those on Fig. (5-4). The comparison shows that the incident electrons from the background slightly affect the charge-state distribution and the fluid profiles for these CIT plasma conditions. For TEXT and low-density TFTR devices, however, the external plasma electrons are even more important than the vapor electrons [56].

The predictions of the channel flow nonequilibrium model shown in Fig. (5-6) are compared with those obtained from the basic model Fig. (5-4). The channeling effect prevents the density from falling off as fast as it would for a spherical expansion. This makes a denser ablation cloud and increase the ionization rate due to the vapor electrons. Comparing the charge-state distribution on the same figures (5-4) and (5-6), one can see that the locations of the higher charge states (CII, CIII, CIV) are moved closer to the 
pellet. The other effect of the constricted fluid (channelling) is the appearance of a shock front followed by a subsonic flow region and Mach one surface. In treating the shock; the point where $d v / d r=0$ is located and then the jump conditions, relating the flow variables on each side of the shock front, are applied. The predictions of channel flow model in Fig. (5-6) show that beyond the shock, the flow proceeds at subsonic speed decelerating further for few sonic radii (while the channel continue to expand) and then accelerating toward Mach one where the channel cross-section is constant. In this postshocked subsonic region the flow decelerates due to the change of the area and accelerates because of the heating effect. If we assume that the area is constant and neglect the atomic processes in this region, then the flow resembles the classic problem of one dimensional flow in a constant area duct with heat interaction [58]. Under these conditions, heat transfer to the ablatant accelerates its flow toward $M=1$ and when this limiting Mach number is reached the flow is said to be thermally choked. Classically, the singularity in the flow $(M=1)$ occurs at the exit from the duct when the background pressure equals the exit pressure where the mean free path is much less than the characteristics length, $r_{p}$; this is certainly not the case here, where the plasma ions have a slowing down length of several centimeters. The channel flow model predictions shows that within 5\% of Mach number unity, the predictions of the code for the Mach number show small oscillations and the equations evince a singularity thereafter. A solution to this problem is given in the next chapter. 
Since the complete model combines the channel flow model and the plasma electrons model, one can see that the complete model predictions shown in Fig. (5-7) are almost identical to those obtained from the channel flow model and shown in Fig. (5-6) because the ionization length for the vapor electrons is much shorter than that for the external plasma electrons for the CIT case. 


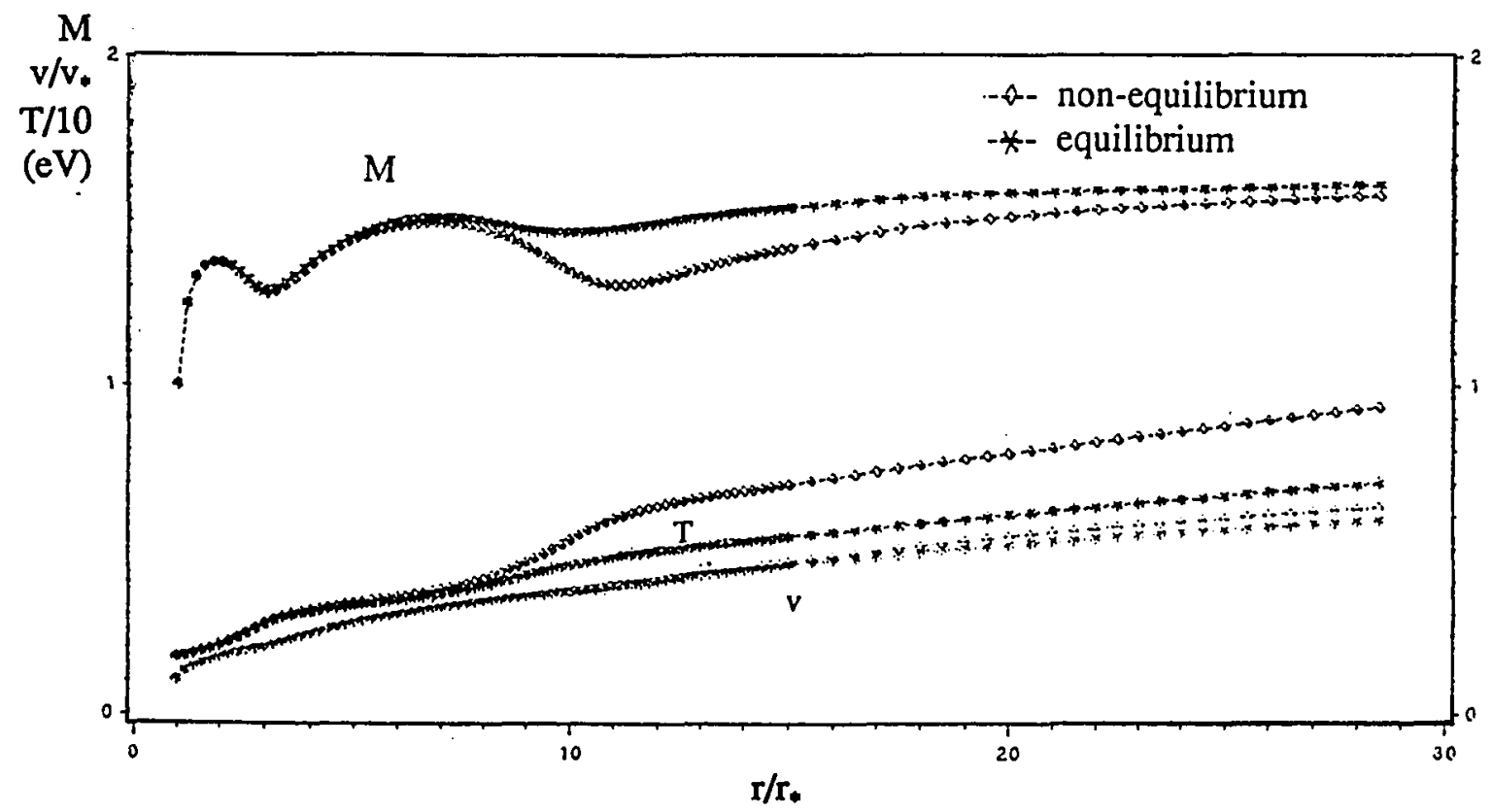

(a) $\log _{10} \mathrm{n}$

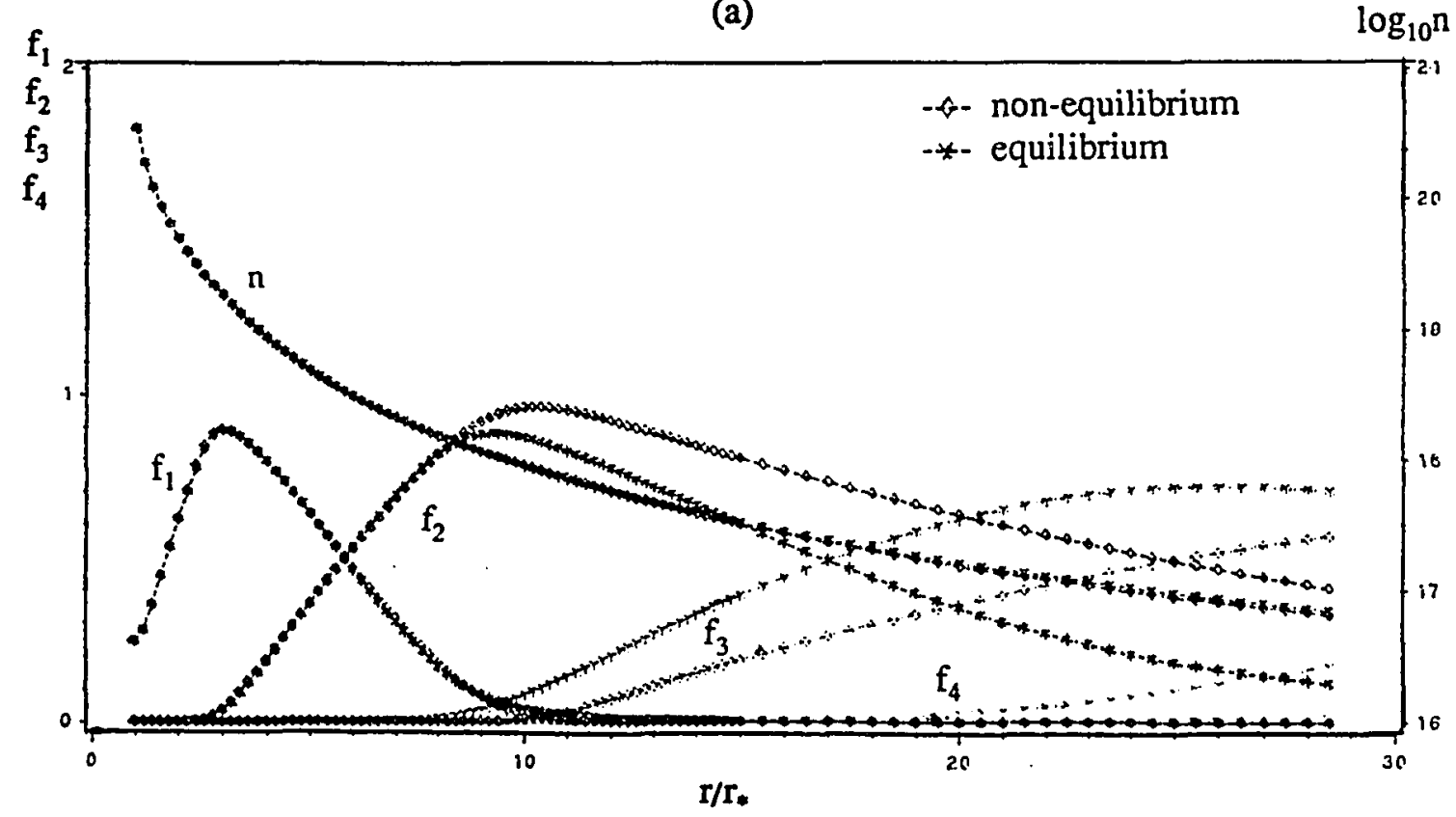

(b)

Fig. 5-1 Comparison of a) Mach number $M$, flow velocity $v$, and cloud temperature $T$, b) total cloud number density $n$ and charge-state fractions $\left(f_{1}, f_{2}, f_{3}, f_{4}\right)$ for equilibrium and non-equilibrium basic models. The pellet radius is $0.05 \mathrm{~cm}$, and the background plasma temperature and density are $20.0 \mathrm{keV}$ and $1.0 \times 10^{14} \mathrm{~cm}^{-3}$, respectively. $r_{*}=0.067$ $\mathrm{cm}$ and $v_{*}=5.3 \times 10^{5} \mathrm{~cm} / \mathrm{s}$. 
M
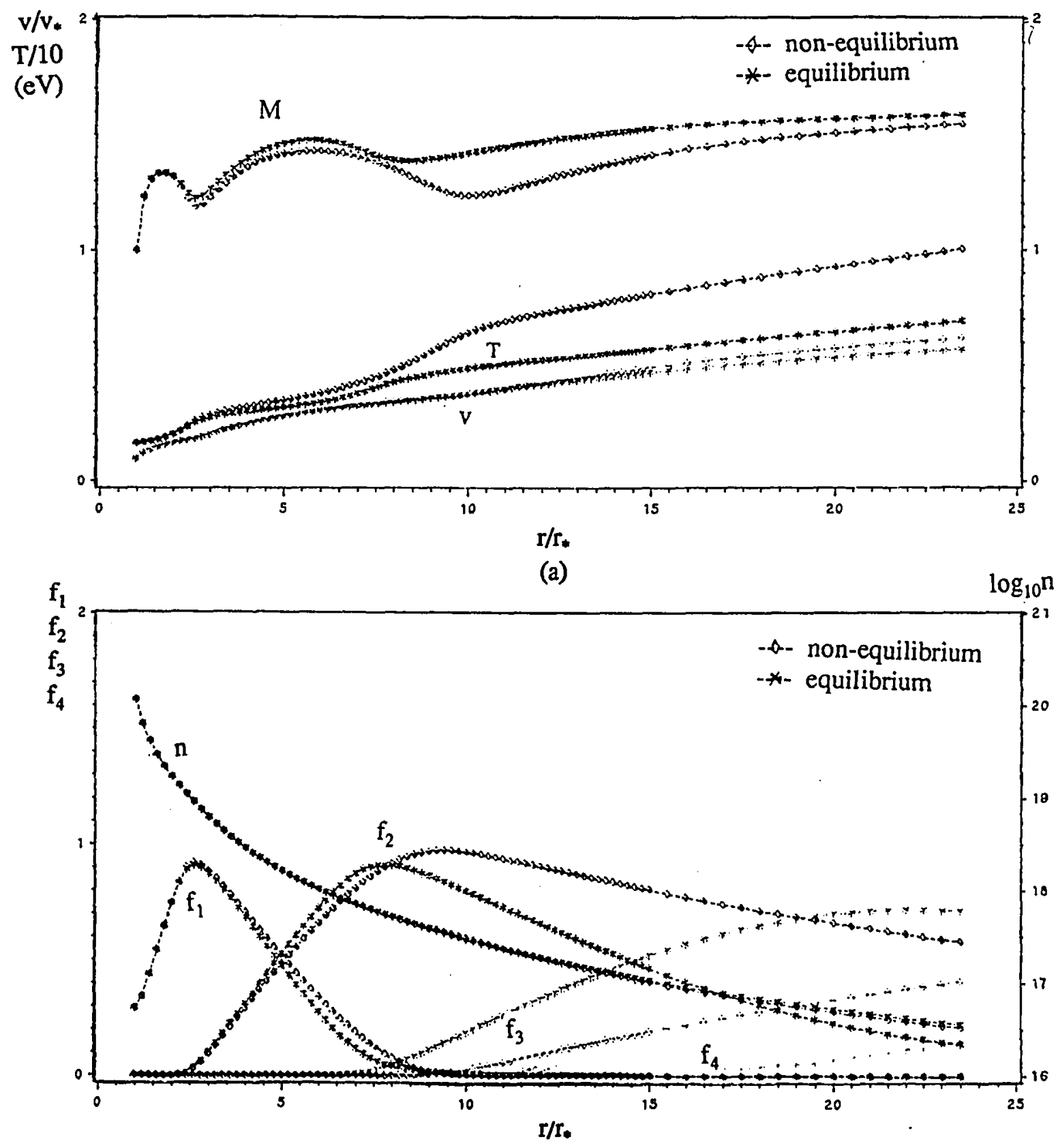

(b)

Fig. 5-2 Comparison of a) Mach number $M$, flow velocity $v$, and cloud temperature $T$, b) total cloud number density $n$ and charge-state fractions $\left(f_{1}, f_{2}, f_{3}, f_{4}\right)$ for equilibrium and non-equilibrium basic models. The pellet radius is $0.05 \mathrm{~cm}$, and the background plasma temperature and density are $10.0 \mathrm{keV}$ and $1.0 \times 10^{14} \mathrm{~cm}^{-3}$, respectively. $r_{*}=0.067$ $\mathrm{cm}$ and $v_{*}=5.31 \times 10^{5} \mathrm{~cm} / \mathrm{s}$. 

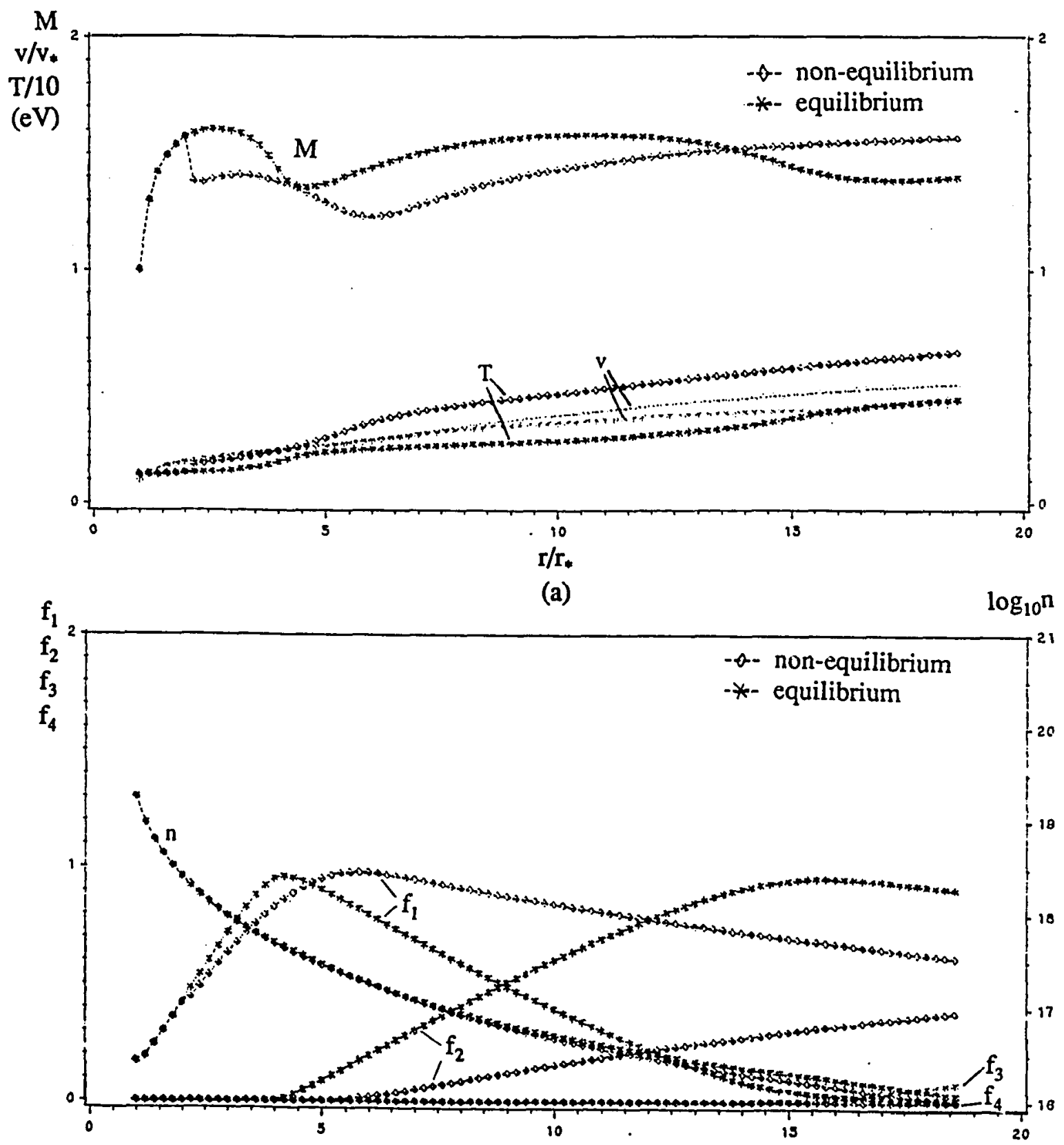

(b)

Fig. 5-3 Comparison of a) Mach number $M$, flow velocity $v$, and cloud temperature $T$, b) total cloud number density $n$ and charge-state fractions $\left(f_{1}, f_{2}, f_{3}, f_{4}\right)$ for equilibrium and non-equilibrium basic models. The pellet radius is $0.025 \mathrm{~cm}$, and the background plasma temperature and density are $3.0 \mathrm{keV}$ and $5.0 \times 10^{13} \mathrm{~cm}^{-3}$, respectively. $\mathrm{r}_{*}=0.033$ $\mathrm{cm}$ and $v_{*}=4.38 \times 10^{5} \mathrm{~cm} / \mathrm{s}$. 


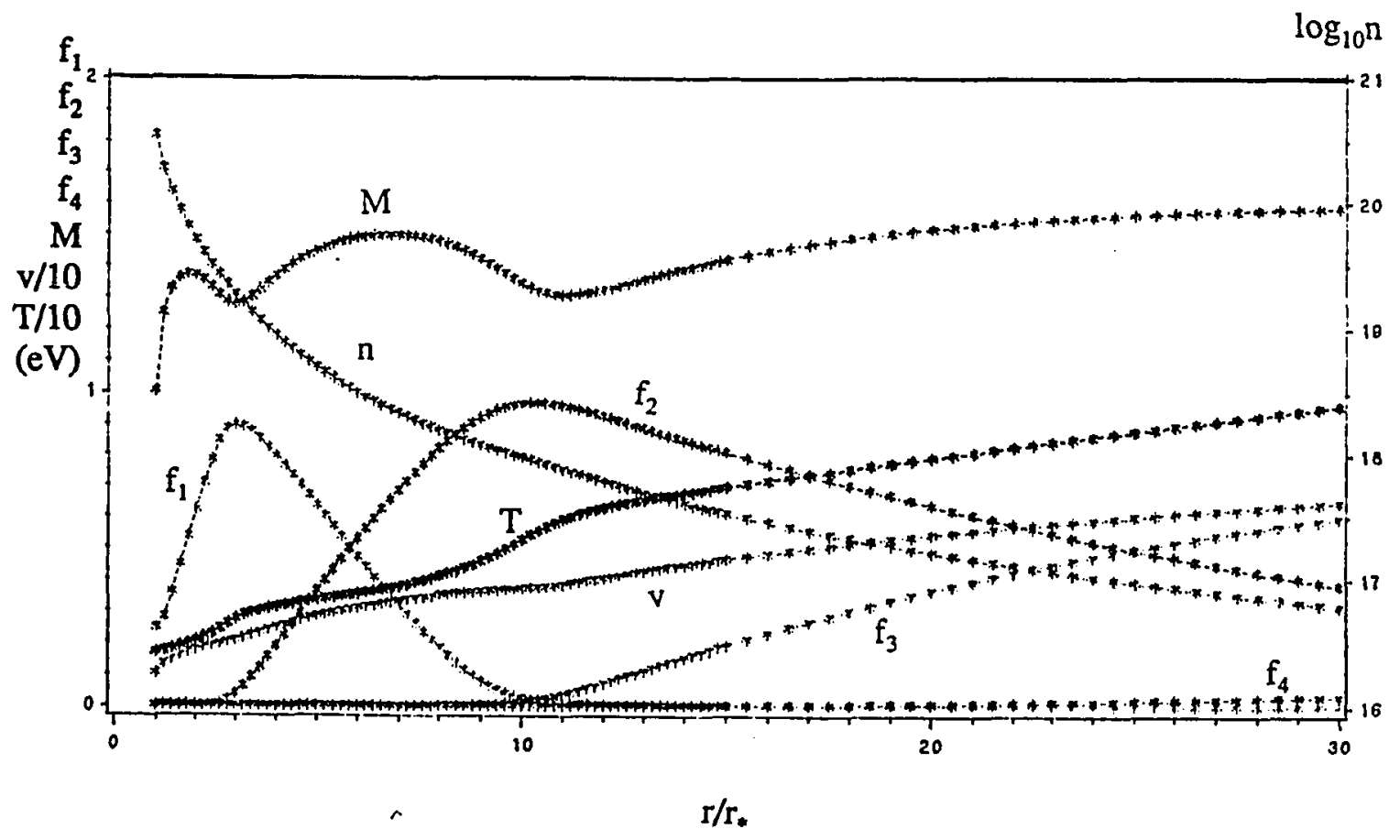

Fig. 5-4 The predictions of the basic model of the Mach number $M$, flow velocity $v$, total cloud number density $n$, cloud temperature $T$, and charge-state fractions $\left(f_{1}, f_{2}, f_{3}\right.$, $\mathrm{f}_{4}$ ). The pellet radius is $0.05 \mathrm{~cm}$, and the background plasma temperature and density are $20.0 \mathrm{keV}$ and $1.0 \times 10^{14} \mathrm{~cm}^{-3}$, respectively. 


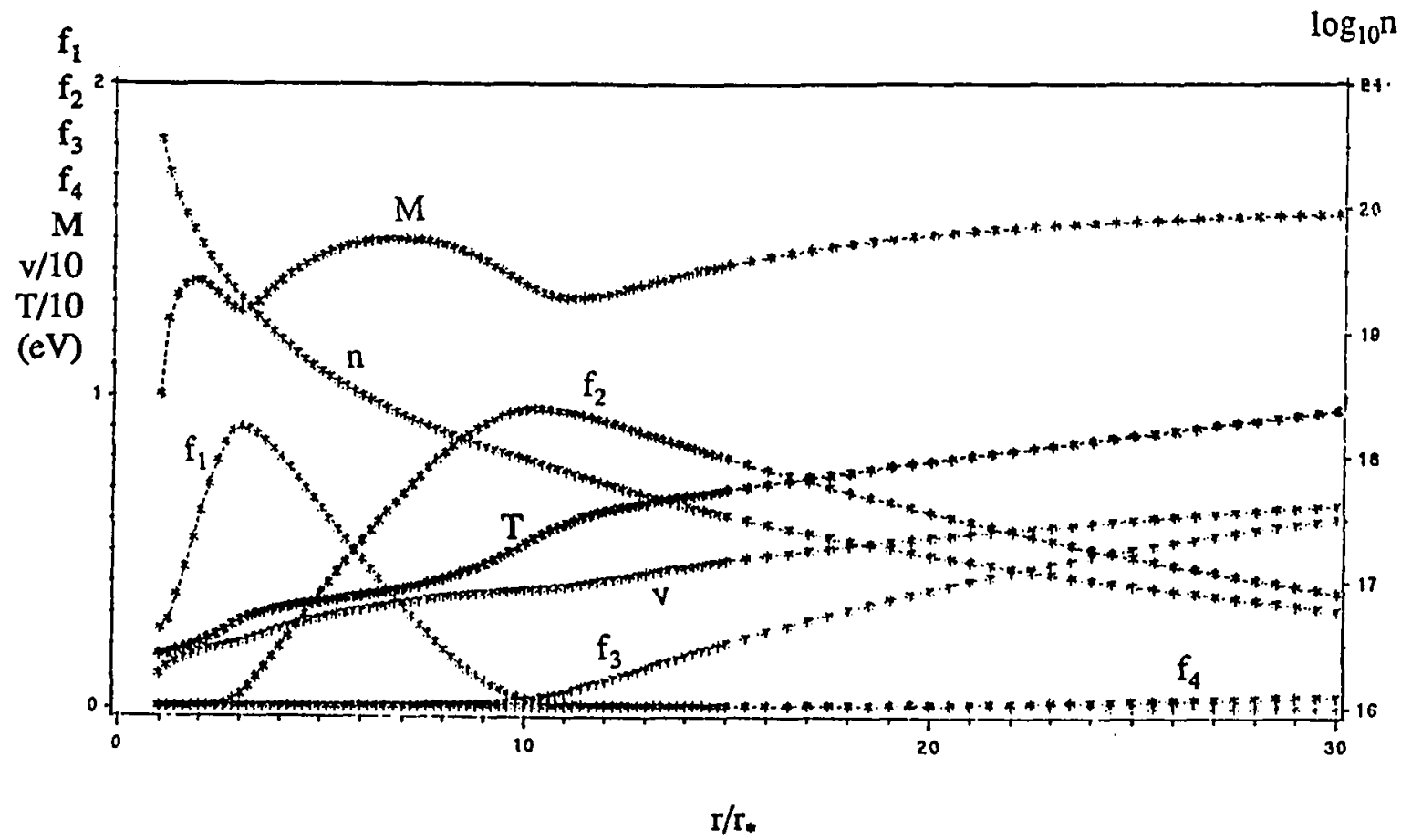

Fig. 5-5 The predictions of the plasma electrons model of the Mach number $M$, flow velocity $v$, total cloud number density $n$, cloud temperature $T$, and charge-state fractions $\left(f_{1}, f_{2}, f_{3}, f_{4}\right)$. The pellet radius is $0.05 \mathrm{~cm}$, and the background plasma temperature and density are $20.0 \mathrm{keV}$ and $1.0 \times 10^{14} \mathrm{~cm}^{-3}$, respectively. 


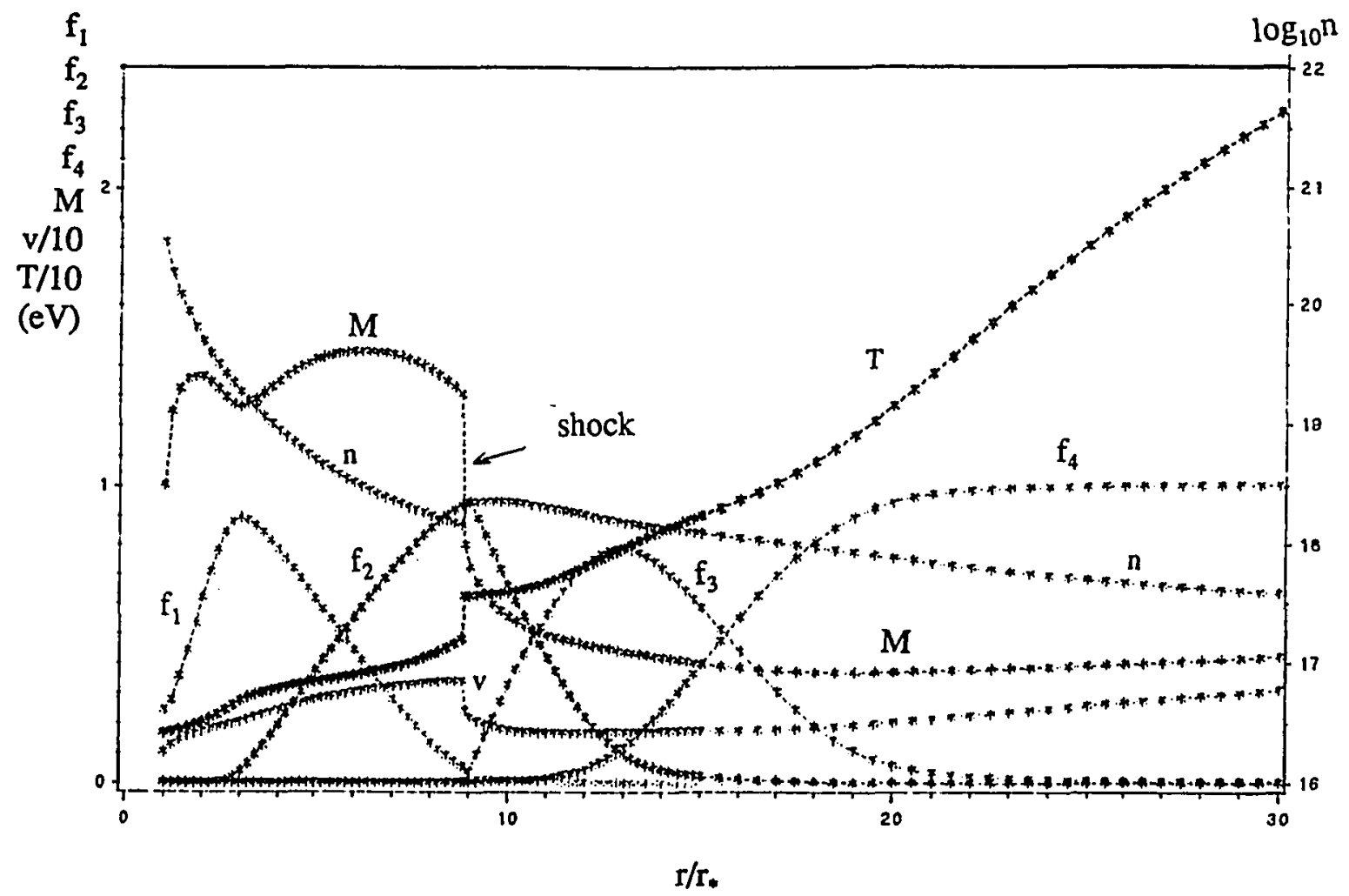

Fig. 5-6 The predictions of the channel flow model of the Mach number $M$, flow velocity $v$, total cloud number density $n$, cloud temperature $T$, and charge-state fractions $\left(f_{1}, f_{2}, f_{3}, f_{4}\right)$. The pellet radius is $0.05 \mathrm{~cm}$, and the background plasma temperature and density are $20.0 \mathrm{keV}$ and $1.0 \times 10^{14} \mathrm{~cm}^{-3}$, respectively. The channel half-width is $25 \mathrm{r}_{*}$, and Eq. (5-6) is used for the area. 


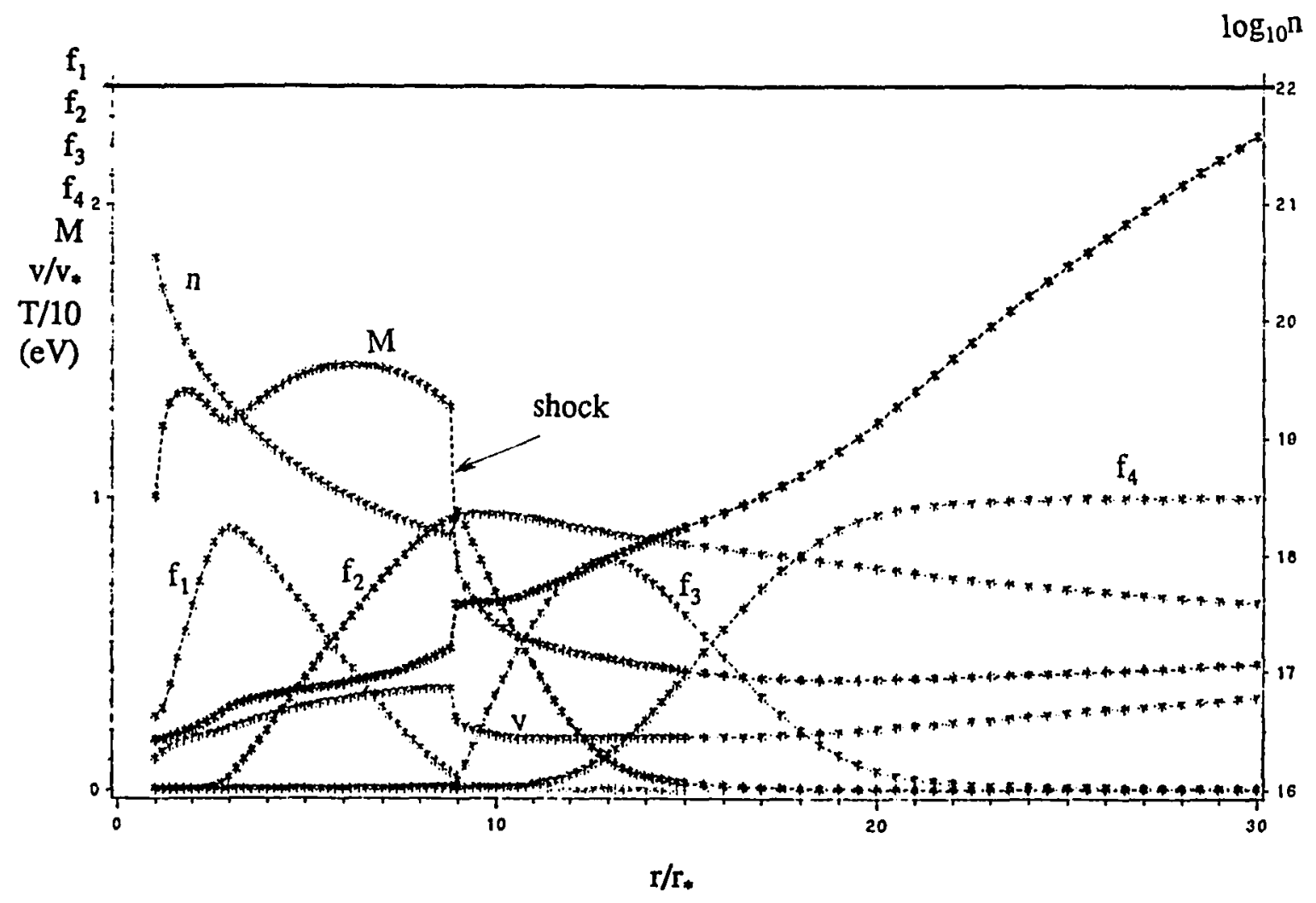

Fig. 5-7 The predictions of the complete model of the Mach number M, flow velocity $v$, total cloud number density $n$, cloud temperature $T$, and charge-state fractions $\left(f_{1}, f_{2}, f_{3}\right.$, $\left.f_{4}\right)$. The pellet radius is $0.05 \mathrm{~cm}$, and the background plasma temperature and density are $20.0 \mathrm{keV}$ and $1.0 \times 10^{14} \mathrm{~cm}^{-3}$, respectively. The channel half-width is $25 \mathrm{r}$, and Eq. (5-6) is used for the area. 


\section{Chapter 6.}

\section{COMPARISON WITH EXPERIMENTAL RESULTS}

\subsection{Introduction}

In the previous chapter, the complete model predictions were limited to a point in the flow at which the Mach number oscillates around $M=1$ and termination of the computation. Similar behavior was observed in theoretical studies of the high-temperature reacting flow. This behavior is briefly discussed as we improve the model to continue its predictions beyond $\mathrm{M}=1$.

In the following section the critical flow model is described. Section 6.3 contain a comparison of the model predictions with experimental results. The pellet penetration is given in section 6.4 .

\subsection{Critical Flow Model}

The assumption of steady state flow implies that the properties of the cloud are dependent on the local background plasma conditions (density and temperature) and hence the shape of the cloud should change gradually as the pellet moves into the plasma. In the low-Z pellet injection experiments on TEXT $[59,60]$, a series of alternately bright and dark regions (striations) occur along the field lines which indicates that the flow may be unsteady. A satisfactory explanation of these striations is not known.

The computational problems associated with high-temperature reacting flow has been investigated by Park [61] for a one-dimensional subsonic flow, through a constant 
area duct, originating behind a normal shock wave. His results show that the chemical reactions induced pressure depressions which form a rarefaction waves and that pressure disturbance was severe for highly ionized gas [61]. Moody in his analyses of small pressure disturbances [62]; shows that the disturbance propagate at the local sound speed if the fluid is stationary. If the disturbance is stationary, the fluid is traveling at velocity equal to the local sound speed, a condition referred to as critical flow, sometimes called choked flow.

The observed striations, the theoretical predictions of the pressure disturbances, and the oscillations predicted by our model seem to be all related to the characteristic of the critical flow. Therefore, it is assumed that within 5\% of Mach one (in the postshocked region) the flow becomes critical and proceed beyond that point with an average velocity equal to the local speed of sound.

To illustrate this argument, let us recall the rules of choking. In an ordinary flow, the Mach number is affected by changes in the flow area, amount of friction, or increase in the heating [58]. For example, in a subsonic flow an increase in the area cause the Mach number to decrease and vice versa. The opposite is true for supersonic flow. If the Mach number unity is reached at some point in the channel, any reduction in the area cannot be introduced without altering the entire flow pattern. This alternation usually is in the form of choking. For the present case, if the channel is filled up with ablation material the heat flux reaching the pellet will be reduced which causes a reduction in the ablation rate. This in turn could reduce the cloud pressure against the magnetic field and resulting in a reduction in the channel area. Therefore, the reduction in the ablation rate 
and area is related to the fluctuations in the line emission from ablating material and the flown pattern alternation.

From this discussion it is to be noted that the flow pattern could be described as a continuous transition through speed of sound. The critical flow model equations, derived in Appendix F, replace the complete model equations, chapter 5, when the Mach number is within $5 \%$ of Mach one.

\subsection{Comparison of the Model Predictions with Experiment}

With these modifications the model can be used to compare its predictions with TEXT experimental results [64]. Typical example of the model's predictions (complete model and critical flow model) are shown in Fig. (6-1). Zero ionization initial conditions are used since the equilibrium criterion is violated. The critical flow model is switched at $M=1$ and the channel half-width, $r_{c h}$, is taken $20 r_{* .}$ The ion density profiles of $C+1$, $C+2$, and $C+3$ and the channel radius are shown in Fig. (6-2). Up to $r \sim 40$ r. the profiles show the same trend described in the previous chapter. For $r>40 \mathrm{r}_{*}$, the predictions of the critical flow model are shown. One sees that the charge-state fraction are increasing at a slower rate as compared to the CIT case in chapter 5 . This is due to the lower plasma background. The Mach number is kept constant and one can see that both the flow velocity and the ablatant temperature are monotonically increasing in the critical flow region; this indicates a mutual dependence of these quantities.

The predictions of the model for various background densities are shown on Figs. (6-3) and (6-4) for comparison with TEXT experimental results [63]. The experimental 
data are obtained from photographs taken of the pellet cloud at various instants along the pellet's path where a filter has been used to observe the emission of $\mathrm{C}+2$ and $\mathrm{C}+3$ line radiation profiles. The experimental data are the full width half maximum (FWHM) of the line emission clouds of $\mathrm{C}+2$ and $\mathrm{C}+3$, for the distance measured in the direction parallel to the magnetic field $B_{z}$ between the two points at which the light signal falls to $50 \%$ of its maximum. The model predictions are the FWHM, distance between the two points at which the ion density falls to $50 \%$ of its maximum, for $\mathrm{C}+2$ and $\mathrm{C}+3$ ions.

As can be seen in Fig. (6-3), the model tend to predict values of the FWHM that are high except for one data point. Considering the experimental uncertainties, especially in the measurement of density and temperature, the agreement of theory and experiment is quite good for $\mathrm{C}+2$ especially in its scaling with $\mathrm{n}_{\mathrm{e}}$. Moreover, the comparison here is not straight forward because the ion densities are not the same as their corresponding line emission. A direct comparison requires a radiation model which is beyond the scope of the present study.

The FWHM of $\mathrm{C}+3$ predicted by the model is different from the data by a factor of five for the case shown in Fig. (6-4). The disagreement between the theory and experiment is partially due to the reasons mentioned above and could indicate the breakdown of the steady state assumption in the outer region where $\mathrm{C}+3$ exit. Another concern is whether the predictions are consistent with the assumptions used in formulating the model. Namely, the cloud heating solely by the electron heat flux. This is related to the flow pattern being steady, since the ablation cloud thickness and the external electron 
heat flux are dependent (see eqn. B11). The unsteady flow effects are well beyond the scope of the present model.

The predictions of the model for a thermonuclear plasma case are shown in Figs. (6-5) and (6-6). As can be seen in Fig. (6-6), the model predicts a very large region of $C+4$. The cloud thickness fall within the range required for the alphas diagnostics $\left(>10^{16}\right.$ $\mathrm{cm}^{-2}$, which is an encouraging result.

\subsection{Pellet Penetration}

The penetration depth of an injected pellet into plasma depends on the injection velocity, pellet size, and material. To control the level of density perturbation, the pellet size should be small. Small pellets, however will require high injection velocities. The rate of decrease of pellet radius as given by eqn. (22) of ref. 6 is

$$
\dot{r}_{p}=\frac{\langle q\rangle}{n_{0}\left(\Delta H+2.7 T_{1}\right)},
$$

where $<q>$ is the average heat flux on the pellet surface, $n_{0}$ is the solid density, $\Delta H$ is the sublimation energy, and $T_{1}$ is the temperature at the pellet surface. For a given plasma profiles one can calculate the penetration depth for a given pellet size using the above equation. The low-Z model [6] should be used to evaluate $\langle q\rangle$ at each point on the pellet trajectory. Vahala et.al. [56] compared the low-Z model [6] predictions for pellet penetration with pellet injection experimental results. The good agreement reported [56] shows that the low- $Z$ assumptions [6] and the modified low- $Z$ assumptions are good approximations. 
As an example, consider the ITER plasma. The temperature and density at the center are $16.7 \mathrm{keV}$ and $1.65 \times 10^{14} \mathrm{~cm}^{-3}$. The minor radius a is $2.2 \mathrm{~m}$ and the temperature and density profiles are of the form $T_{e}=T_{e 0}\left(1-x^{2}\right)$ and $n_{c}=n_{e 0}\left(1-x^{2}\right)^{1 / 2}$, where $x$ is the distance from the plasma center normalized to the minor radius, $x=r / a$. Fig. 6-7 shows the penetration depth measured from the plasma edge versus the injection velocity for a carbon graphite pellet of initial radius $0.14 \mathrm{~cm}$. For the pellet to reach the plasma center, the required injection velocity should be $26.5 \mathrm{Km} / \mathrm{s}$. Fig. $6-8$ shows the variation of the pellet size as it enters the plasma at $x=1$ and completely ablate at $x=0.37$. The injection velocity for this case is $10 \mathrm{Km} / \mathrm{s}$. On the same Fig. $6-8$, the temperature and density profiles show the corresponding plasma background seen by the pellet. From Fig. 6-7, a carbon graphite pellet of initial radius $0.14 \mathrm{~cm}$ requires an injection velocity of $\sim 5 \mathrm{Km} / \mathrm{s}$ to reach half-way into the plasma, $x=1 / 2$. Two-stage gas gun has achieved almost 5 $\mathrm{Km} / \mathrm{s}$ [64]. Hence, it appears that the pellet could reach halfway to the center. Since, the density of the fast alphas will be within a factor of two of the peak value, Fig. 4 of Gerdin [18], a useful information on their energy distribution could be obtained. 

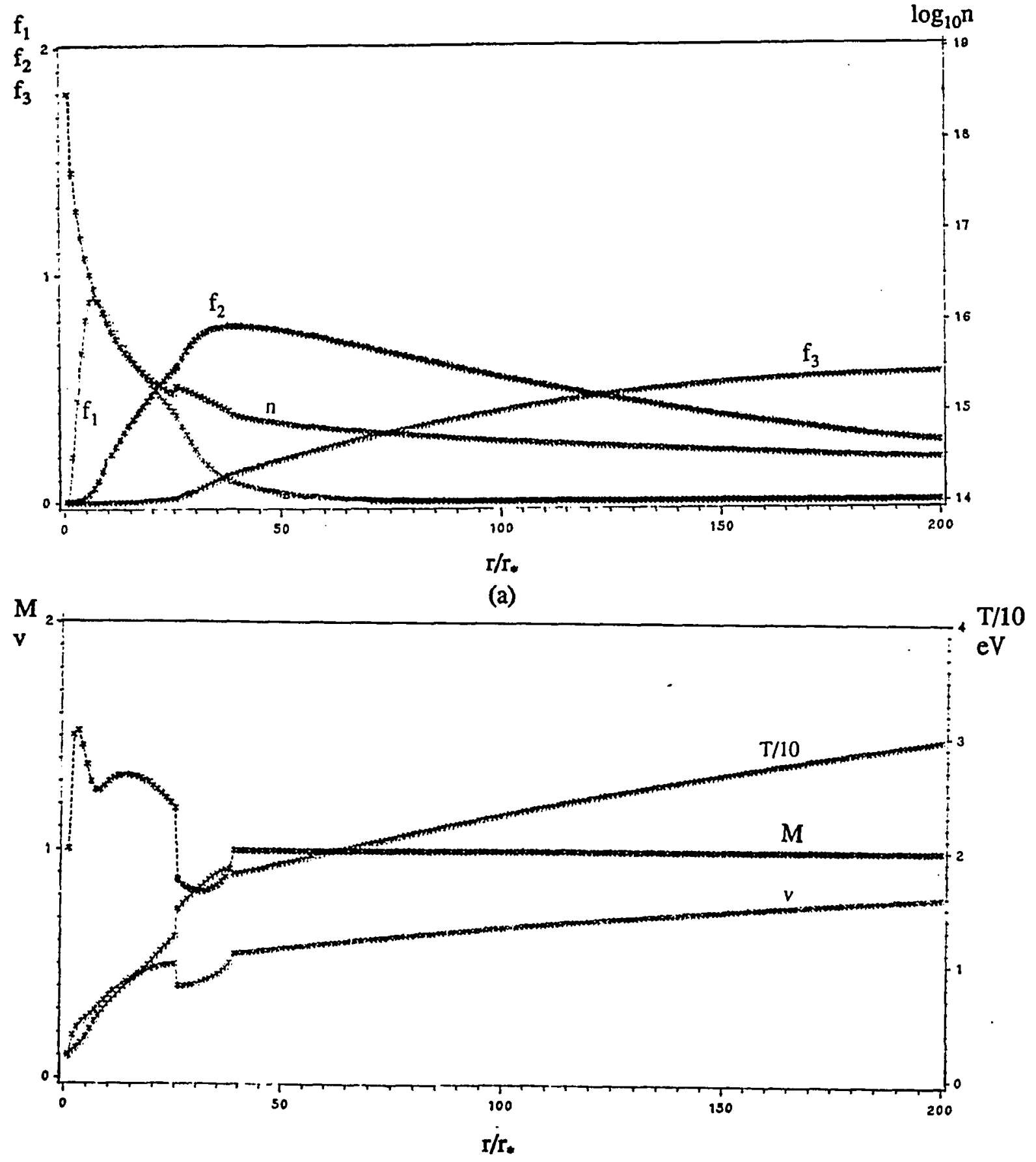

(b)

Fig. 6-1 The predictions of the complete model and critical flow model for a typical TEXT case: a) charge state fraction and total number density, b) Mach number, flow velocity and cloud temperature. The pellet radius is $0.03 \mathrm{~cm}$, and the background plasma temperature and density are $1.0 \mathrm{keV}$ and $5.0 \times 10^{13} \mathrm{~cm}^{-3}$, respectively. The channel halfwidth is $20 \mathrm{r}_{*}$, and Eq. (5-26) is used for the area. $r_{*}=0.038 \mathrm{~cm}$ and $v_{*}=4.93 \times 10^{5}$ $\mathrm{cm} / \mathrm{s}$. 


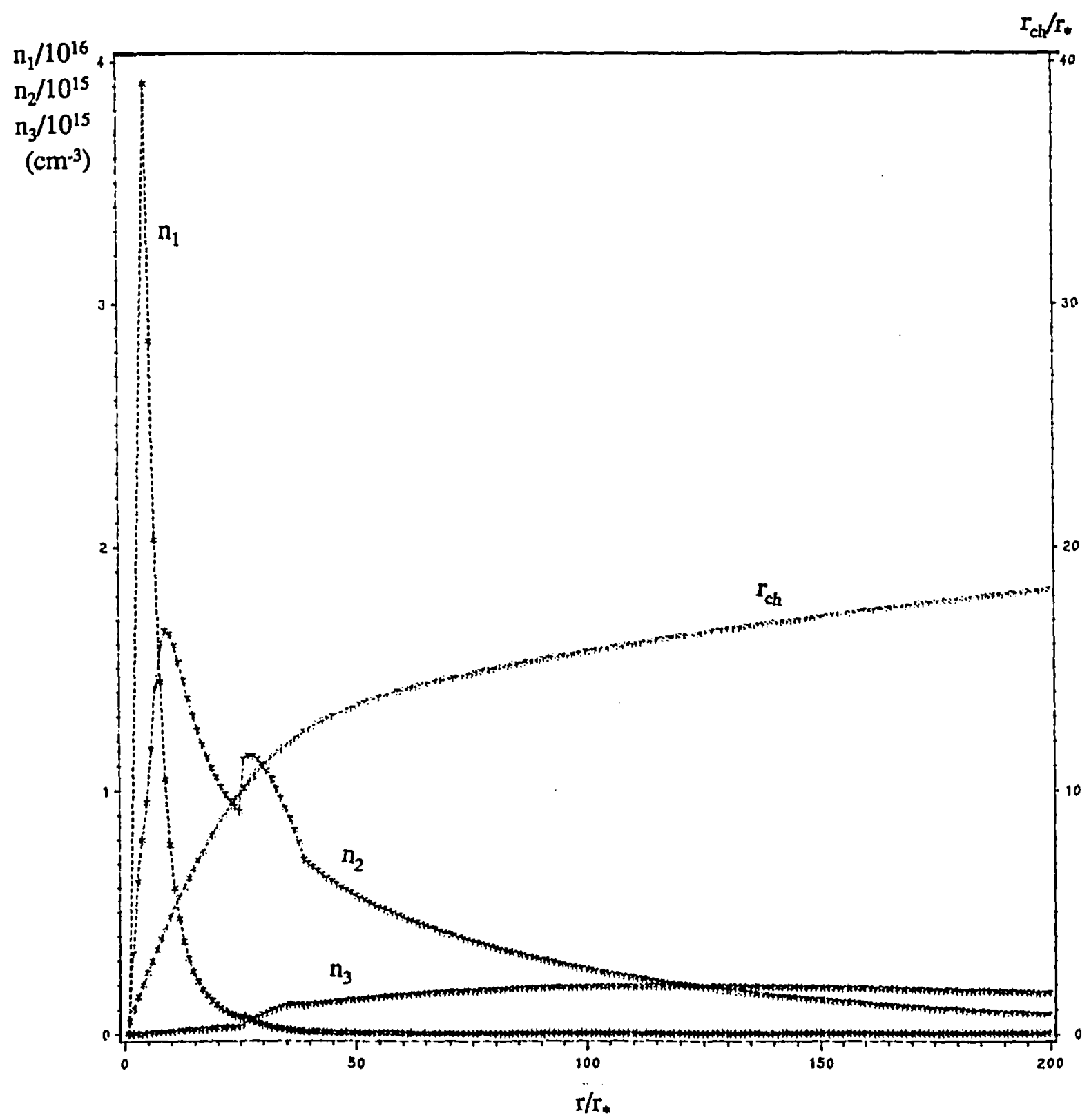

Fig. 6-2 The predictions of the complete model and critical flow model for a typical TEXT case. The pellet radius is $0.03 \mathrm{~cm}$, and the background plasma temperature and density are $1.0 \mathrm{keV}$ and $5.0 \times 10^{13} \mathrm{~cm}^{-3}$, respectively. The channel half-width is $20 \mathrm{r}$, and Eq. $(5-26)$ is used for the area. $r_{*}=0.038 \mathrm{~cm}$ and $v_{*}=4.93 \times 10^{5} \mathrm{~cm} / \mathrm{s}$. 
FWHM (cm)

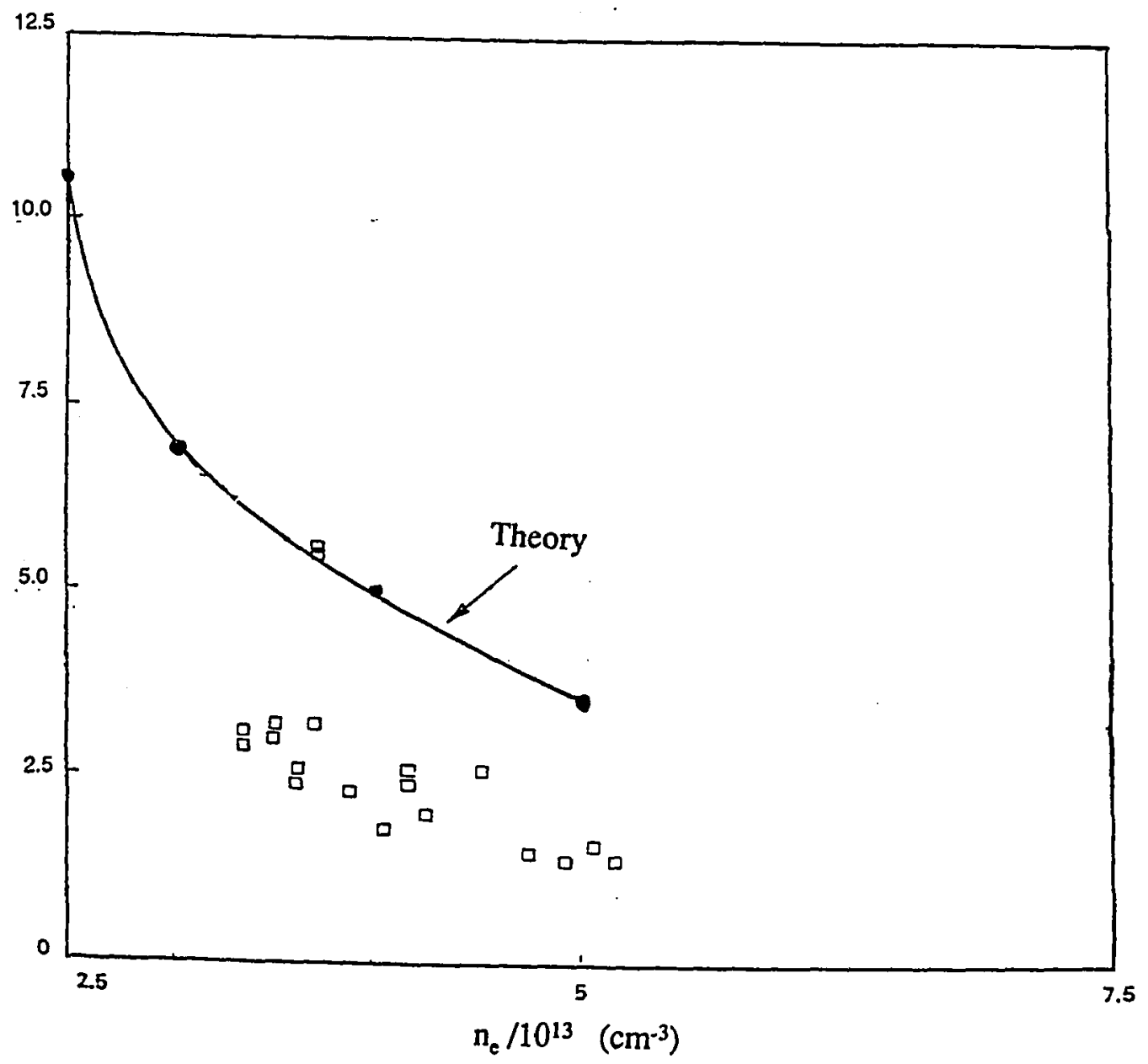

Fig. 6-3 Comparison of the predictions of theory with the data from TEXT for $\mathrm{C}^{+2}$. The pellet radius is $0.03 \mathrm{~cm}$ and the background plasma temperature is $1.0 \mathrm{keV}$. 


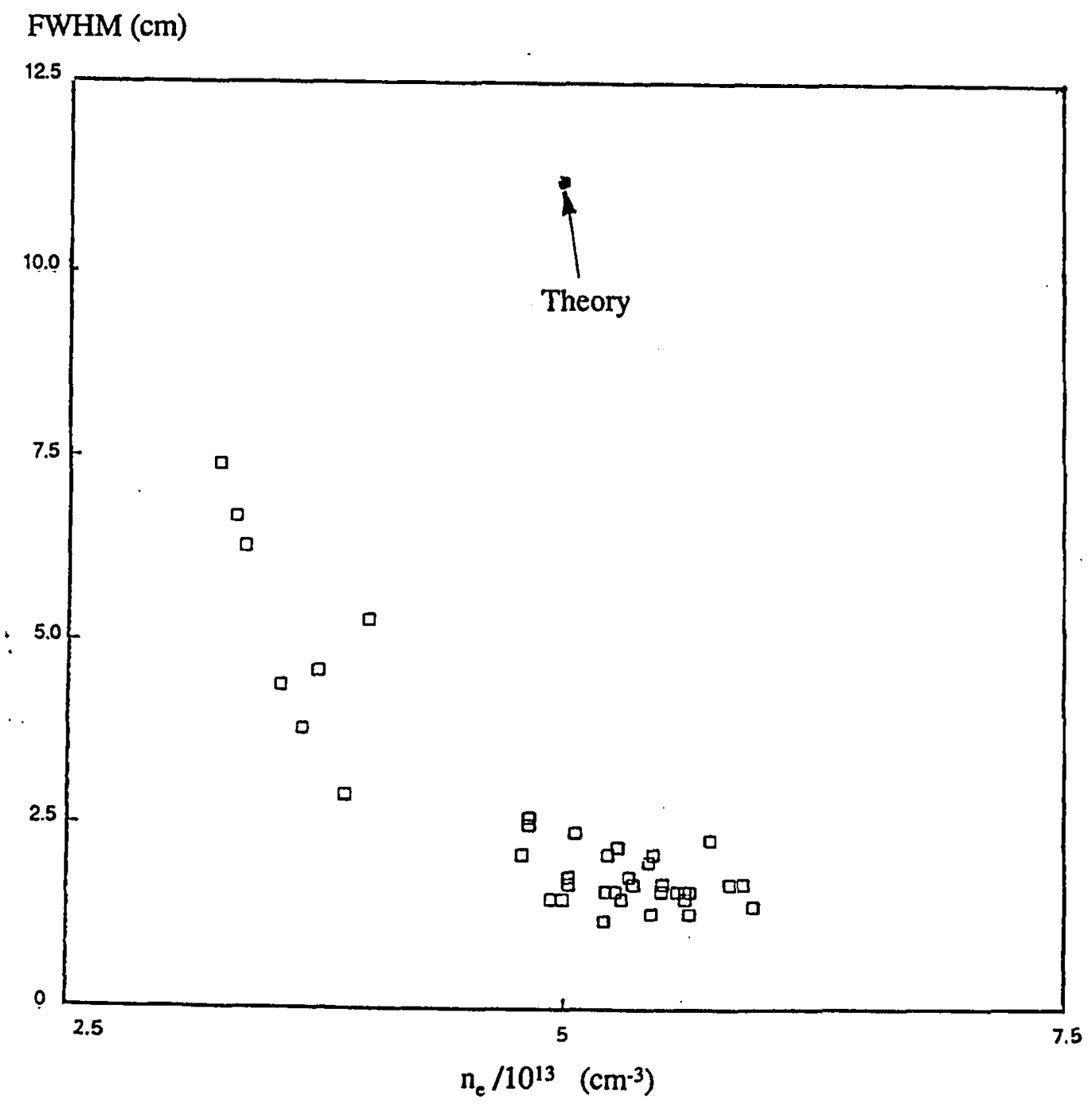

Fig. 6-4 Comparison of the predictions of theory with the data from TEXT for $\mathrm{C}^{+3}$. The pellet radius is $0.03 \mathrm{~cm}$ and the background plasma temperature is $1.0 \mathrm{keV}$. 

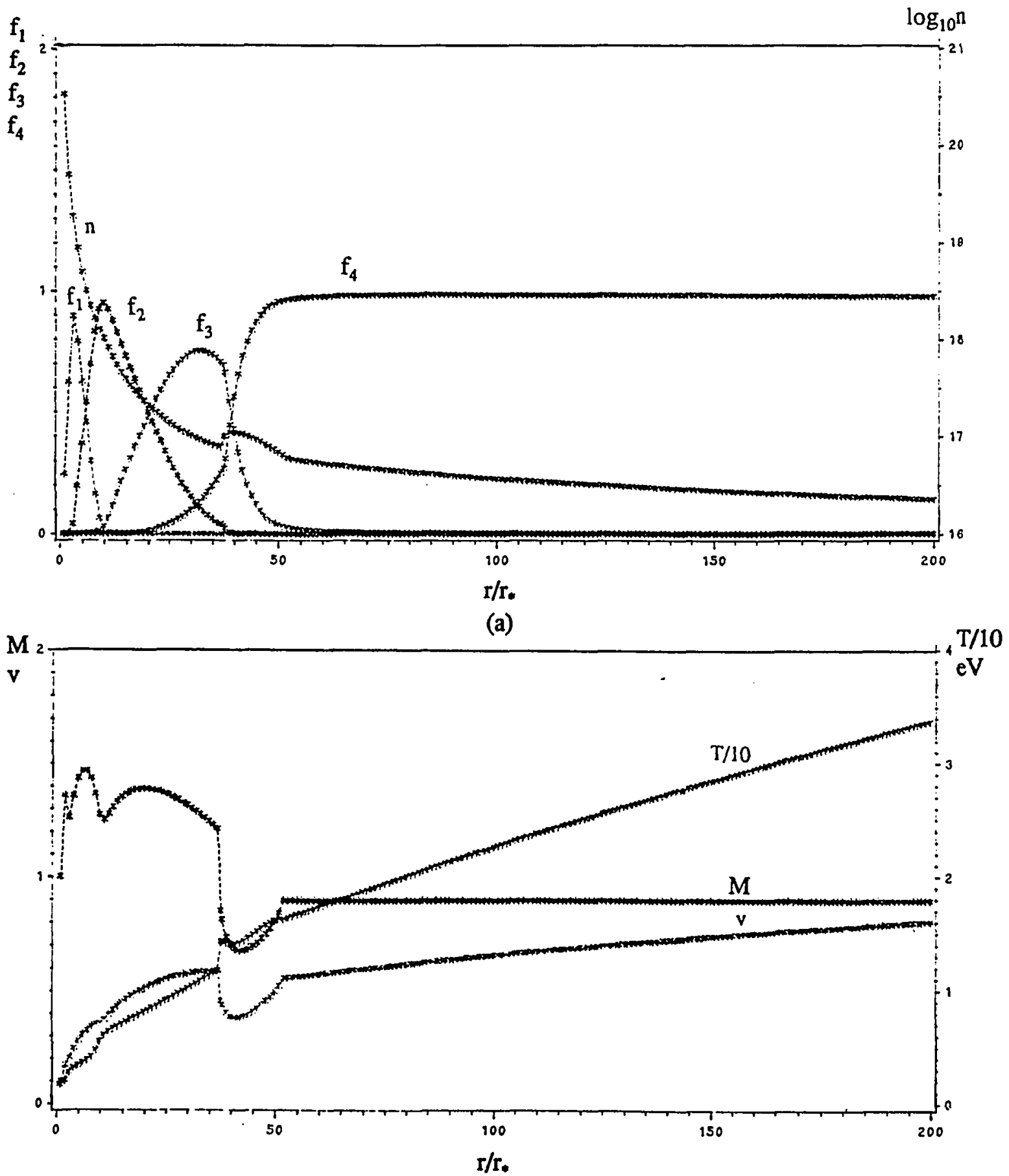

(b)

Fig. 6-5 The predictions of the complete model and critical flow model for a typical CIT case: a) charge state fraction and total number density, b) Mach number, flow velocity and cloud temperature. The pellet radius is $0.05 \mathrm{~cm}$, and the background plasma temperature and density are $20.0 \mathrm{keV}$ and $1.0 \times 10^{14} \mathrm{~cm}^{-3}$, respectively. The channel halfwidth is $20 r_{*}$, and Eq. $(6-16)$ is used for the area. $r_{*}=0.067 \mathrm{~cm}$ and $v_{*}=5.3 \times 10^{5} \mathrm{~cm} / \mathrm{s}$. 


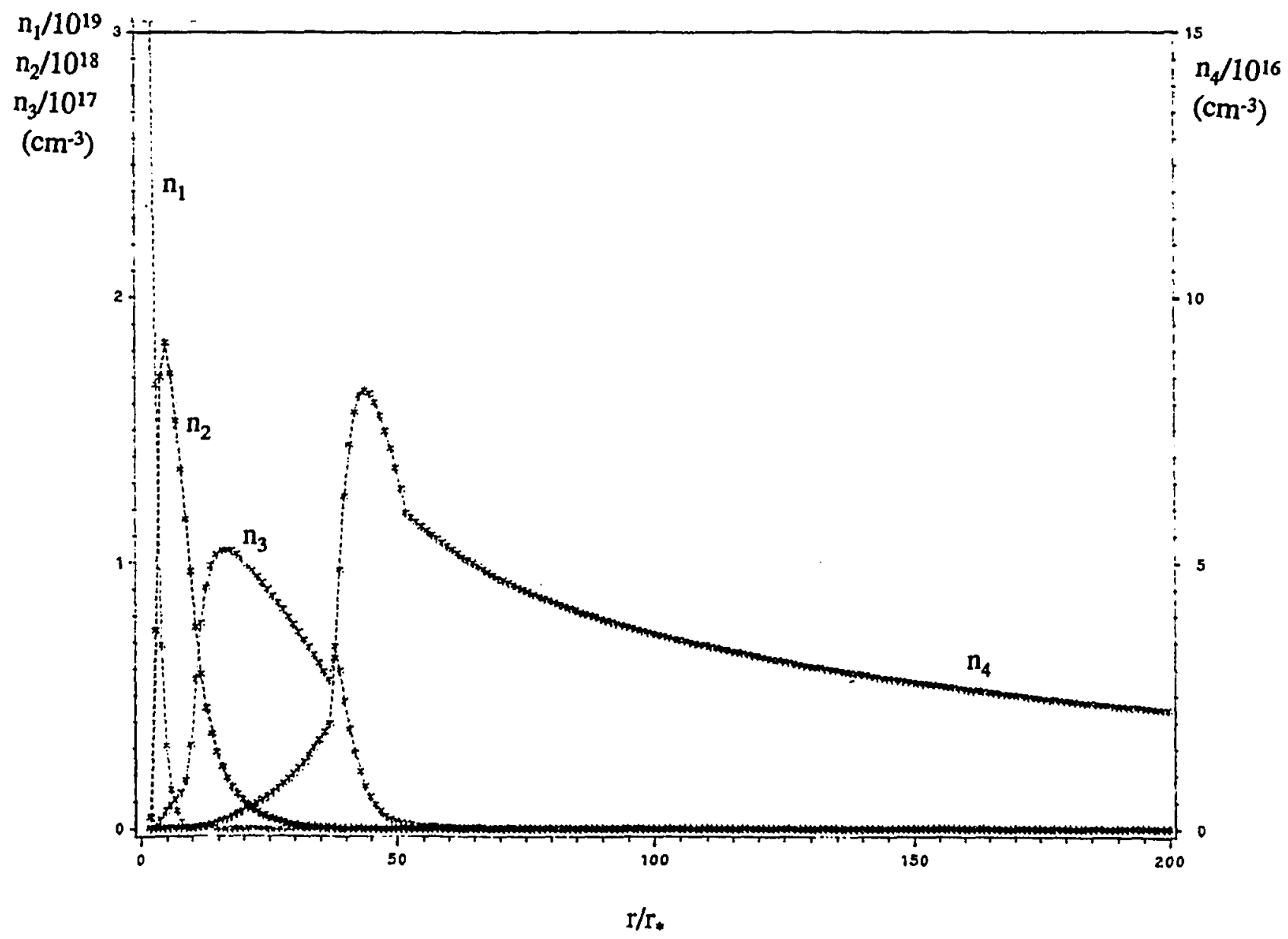

Fig. 6-6 The predictions of the complete model and critical flow model for a typical CIT case. The pellet radius is $0.05 \mathrm{~cm}$, and the background plasma temperature and density are $20.0 \mathrm{keV}$ and $1.0 \times 10^{14} \mathrm{~cm}^{-3}$, respectively. The channel half-width is $20 \mathrm{r}$, and Eq. $(6-16)$ is used for the area. $r_{*}=0.067 \mathrm{~cm}$ and $v_{*}=5.3 \times 10^{5} \mathrm{~cm} / \mathrm{s}$. 


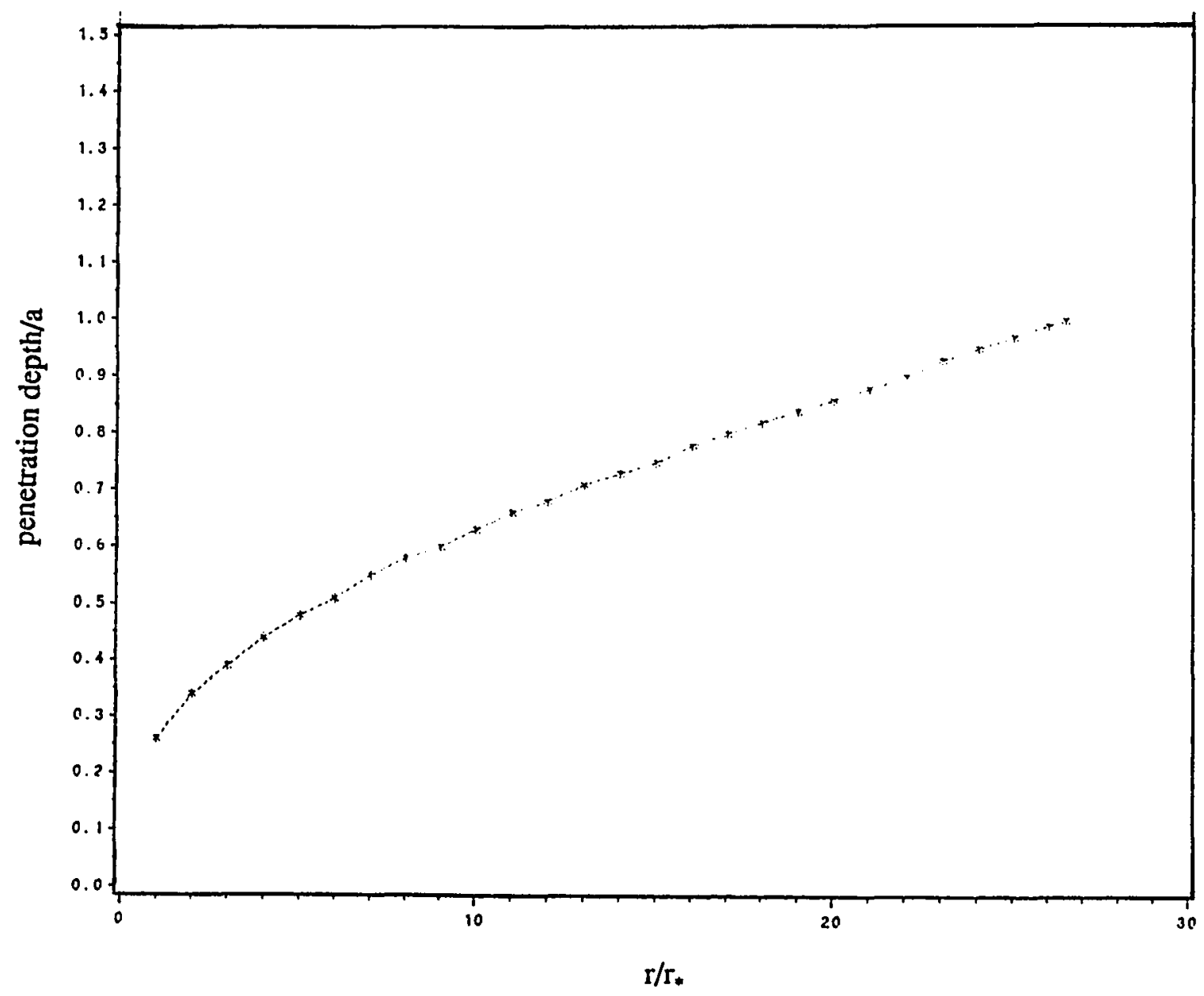

Fig. 6-7 The penetration depth measured from plasma edge versus the pellet velocity. The pellet radius is $0.14 \mathrm{~cm}$, and the background tempreature and density profiles are of the form $T_{c}=T_{e 0}\left(1-x^{2}\right)$ and $n_{c}=n_{c 0}\left(1-x^{2}\right)$ with $T_{e 0}=16.7 \mathrm{keV}$, and $n_{c 0}=1.65 \times 10^{14}$ $\mathrm{cm}^{-3}$. 


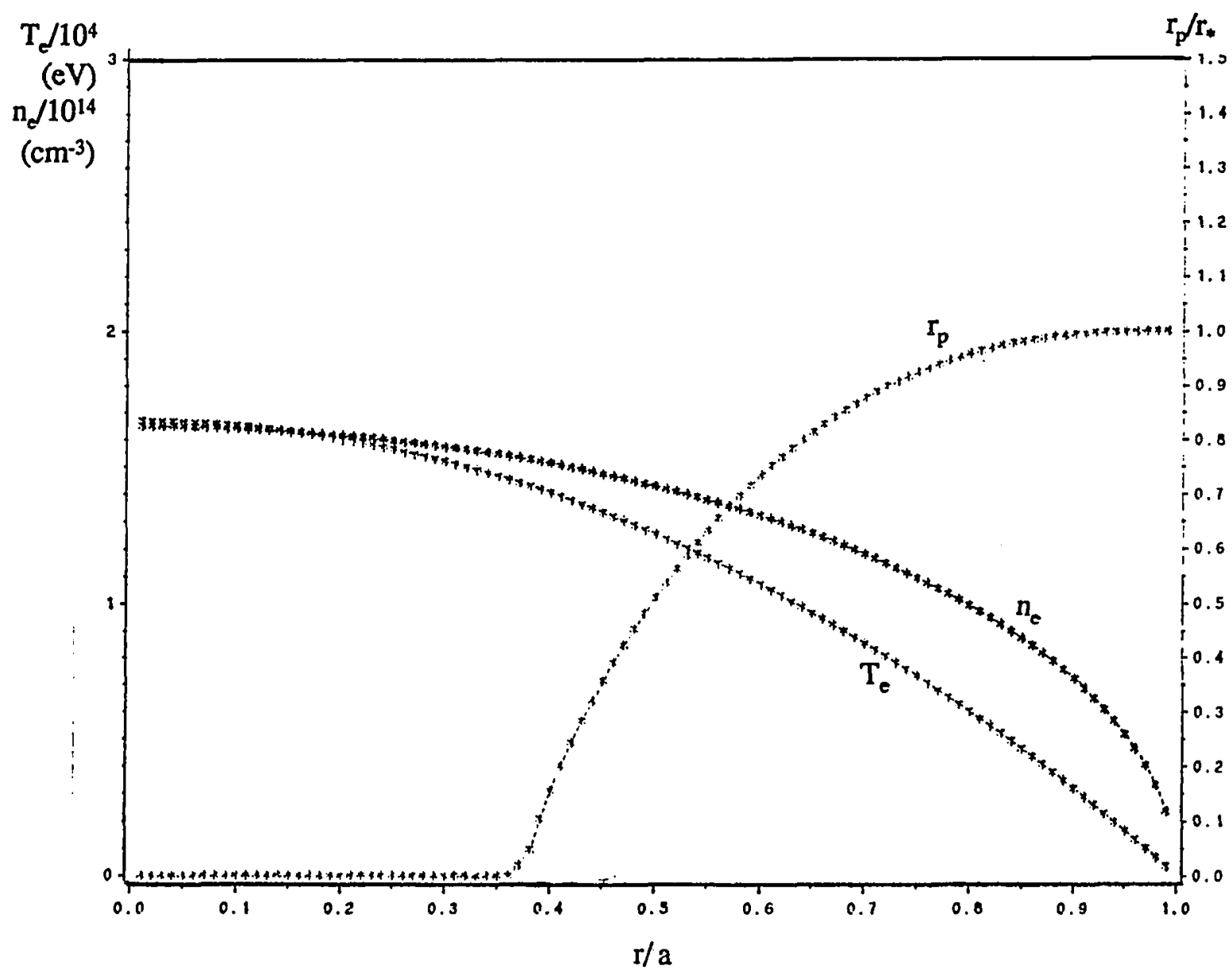

Fig. 6-8 Radius of a carbon pellet versus the normalized minor radius ( $r / a)$. The pellet velocity is $10 \mathrm{~km} / \mathrm{s}$. Also shown are the preinjection profiles for the background temperature and density. 


\section{Chapter 7.}

\section{CONCLUSIONS AND RECOMMENDATIONS}

\subsection{Conclusions}

In this study we developed a model which can be used to determine the spatial distributions of the ionization states in the ablation cloud resulting from the interaction of pellets with magnetoplasma. The model provide equilibrium and nonequilibrium calculations.

Application of the model to the hydrogen pellets showed that the equilibrium solutions merge smoothly onto those obtained from the nonequilibrium calculations when the ionization time is much shorter than the flow time. A criterion for applying the equilibrium was obtained. This criterion indicates that the nonequilibrium calculations are important because the existing experimental devices such as TEXT and TFTR operates in a region where this criterion is violated. Additionally, the nonequilibrium calculation can be used to predict $\mathrm{H}_{\alpha}$ emission, since this emission is used to determine the penetration depth and material deposition in refueling plasma by deuterium-tritium pellets.

Nonequilibrium effects are even more important in carbon-pellet calculations because in the portion of the cloud that is suitable for alpha particle diagnostics equilibrium criterion will not be satisfied.

Inclusion of the magnetic field effect (channel flow), observed experimentally, is important for agreement with experiment. The good agreement for $\mathrm{C}+2$ between theory 
and experiment indicates the following: (1) the zero ionization initial condition, which is dictated by the equilibrium criterion, is a good approximation for TEXT plasma; (2) the choice of the channel half-width $r_{c h}$ is consistent with experiment; (3) the steady state flow assumption is a good working approximation in the supersonic flow region, and (4) the critical flow model is less restrictive approximation.

\subsection{Recommendations for Further Work}

The large region of $\mathrm{C}+4$ predicted by the model could provide the first opportunity to study the alpha particle physics. Fisher et.al. [65] showed that, the density in the $\mathrm{C}+4$ region of the cloud should be high enough to produce an equilibrium fraction of helium neutrals. They also calculated the upper limt on the ablation cloud density necessary to avoid significant energy loss by the incident alphas due to scattering. Since the perpendicular thickness of the predicted cloud falls in the range set by Fisher et.al. [65], it should be sufficient for alpha diagnostics.

To be more confident, one would desire better agreement between model predictions of $\mathrm{C}+3$ and experiment. This might require the inclusion of ion and/or alpha heating of the ablatant in the outer region and probably radiation as well. However, this is beyond the scope of the present work.

Also, it seems necessary to develop a two-dimensional time-dependent flow model to properly account for the interactions of the ablated material with the magnetic field. This should bring the theory in closer agreement with experiment. 


\section{REFERENCES}

[1] "Presentations From the Workshop on Alpha-particle Diagnostics on CIT", Princeton Plasma Phys. Lab. Rep. AA-870710-PPL-06, Ed. K. M. Yong, 3-5 June, 1987.

[2 ] G. Gerdin and L. Vahala, reference 1.

[3 ] R.K. Fisher, J.S. Leffler, A.M. Howald, P.B. Parks, K.H. Burrel, and W. Heidbrink, reference 1 .

[4 ] E. Marmar, J.L. Terry, B. Lipschultz, and J.E. Rice, a reference 1.

[5 ] M. Sasao, K.N. Sato, Y. Nakamura, and M. Wakatani, Nucl. Fusion 27, 335 (1987).

[6 ] P.B. Parks, J.S. Leffler, and R.K. Fisher, Nucl. Fusion 28, 477 (1988).

[7 ] S.J. Zweben, Rev. Sci. Intstrum., 57, 1723 (1986).

[8 ] D.E. Post, D.R. Mikkelson, R.A. Hulse, L.D. Stewart, and J.C. Wieshet, J. Fusion Energy 1, 129, (1981).

[9] M. Sasao and K.N. Sato, Fusion Technol. 10, 236, (1986).

[10] P.B. Parks, R.J. Turnbull, and C.A. Foster, Nucl. Fusion 17, 539 (1977).

[11] C.A. Foster, R.J. Colchin, S.L. Milora, K. Kim, and R.J. Turnbull, Nucl. Fusion 17, 1067 (1977).

[12] P.B. Parks and R.J. Turnbull, Phys. Fluids 21, 1735 (1978).

[13] S.L. Milora and C.A. Foster, IEEE Trans. Plasma Sci. PS-6, 578 (1978).

[14] C.T. Chang, L.W. Jorgensen, P. Nielson, and L.L. Lengyel, Nucl. Fusion 20, 859 (1980).

[15] S.L. Milora, J. Fusion Energy 1, 15 (1981). 
[16] S.L. Milora, C.A. Foster, C.E. Thomas, C.E. Buch, J.B. Wilgen, E.A. Lazarus, J.L. Dunlap, P.H. Edmons, W.A. Houlberg, H.C. Howe, D.P. Hutchinson, M. Murakami, G.H. Neilson, J.A. Rome, and M.J. Saltmarsh, Nucl. Fusion 20, 1491 (1980).

[17] F.S. Felber, P.H. Miller, P.B. Parks, R. Prater, and D.F. Vaslow, Nucl. Fusion 19, 1061 (1979).

[18] G. Gerdin, Phys. Fluids 30, 3782 (1987).

[19] P.B. Parks, Nucl. Fusion 20, 311 (1980).

[20] Y. Nakamura, H. Nishihara, and M. Wakatani, Nucl. Fusion 26, 907 (1986).

[21] M. Kaufman, K. Lackner, L.L. Legyel, and W. Schneider, Nucl. Fusion 26, 171 (1986).

[22] W.A. Houlberg, S.L. Milora, and S.E. Attenberger, Oak Ridge National Lab., ORNL/TM-10556 (1987).

[23] K.N.C. Bray, in Nonequilibrium Flows Part II, edited by P.P. Wegener, (Marcel Dekker, New York, 1970), chapter 3.

[24] W.G. Vincenti, and C.H. Kruger, Introduction to Physical Gas Dynamics, (John Wiley, New York, 1967).

[25] R.W.P. McWhirter, in Plasma Diagnostic Techniques, edited by R.H. Huddlestone and S.L. Leonard (Academic, New York, 1965), chapter 5.

[26] D. Mihalas, "Stellar Atomspheres" (W.H. Freeman, San Francisco, 1978).

[27] D.R. Bates, A.E. Kingston, and R.W.P. McWhirter (1962 a), Proc. R. Soc. London, Ser. A 267, 297.

[28] D.R. Bates, A.E. Kingston, and R.W.P. McWhirter (1962 b), Proc. R. Soc. London, Ser. A 270, 155.

[29] D.R. Bates, and A.E. Kingston, (1963 b), Planet Space Sci. 11, 1.

[30] R.W.P. McWhirter, and A.G. Hearn, (1963), Proc. Phys. Soc. London, 82, 641.

[31] J. Magill, J. Phys. D. 10, p. 2257, 1977.

[32] M. J. Seaton, Planetary and Space Sci., 12 (1964) 55. 
[33] A. Burgess, and I. C. Percival, Adv. At. Mol. Phys., 4, (1968) 109.

[34] A. Burgess, H. P. Summers, Mon. Not. R. Astron. Soc., 174, (1976) 345.

[35] D. H. Sampson, and L. B. Golden, J. Phys. B 11, (1978) 541.

[36] L. B. Golden, D. H. Sampson, J. Phys. B 13, (1980) 2645.

[37] D.E. Post, B.V. Jensen, C.B. Tayrt, W.H. Grasberger, W.A. Lokke, Atomic Data and Nucl. Data Tables 20, 397 (1977).

[38] H.R. Griem, Plasma Spectroscopy, (McGraw-Hill, New York, 1964), chapter 6.

[39] M.J. Seaton, Mon. Not. R. Astr. Soc. 119 (1959) 81.

[40] A. Burgess, and M. J. Seaton, Mon. Not. R. Astron. Soc., 120, (1960) 121.

[41] Y.B. Zeldovich, and Y.P. Raizer, in Physics of Shock Waves and High Temperature Hydrodynamic Phenomena Vol. II, edited by W.D. Hayes and R.F. Probstein, (Academic, New York, 1967).

[42] J.P. Appleton, and K.N.C. Bray, The Conservation Equations for a Nonequilibrium Plasma, J. Fluid Mech. (1964), 20, pp 659-672.

[43] A.Q. Eschenroeder, D.W. Boyer, and J.G. Hall, Nonequilibrium Expansions of air with Coupled Chemical Reactions, Phys. Fluids. 5, 615 (1962).

[44] M.N. Macrossan, The Equilibrium Flux Method for the Calculation of Flows with Nonequilibrium Chemical Reactions, J. Comput. Phys., $\underline{80}, 204$ (1989).

[45] B. Grossman, and P. Cinnella, Flux Split Algorithms for Flows with Nonequilibrium Chemistry and Vibrational Relaxation, J. Comput. Phys., $\underline{88}, 131$ (1990).

[46] A.Q. Eschenroeder, Ionization Nonequilibrium in Expanding Flows, ARS Jour., 32, 196 (1962).

[47] G.V. Candler, and R.W. MacCormack, The Computation of Hypersonic ionized Flows in Chemical and Thermal Nonequilibrium, AIAA Paper No. 88-0511, 1988.

[48] D.M. Thomas, R.K. Fisher, J.S. Leffler, P.B. Parks, S.C. McCool, W.L. Rowan, F.C. Anderson, and M.L. Walker, Bult. Amer. Phys. Soc. 33, 1966 (1988). 
[49] M.L. Walker, F.C. Anderson, S.C. McCool, K.R. Carter, T.K. Herman, and E. Marmar, Bult. Amer. Phys. Soc. 33, 2024 (1988).

[50] S.E. Attenberger, W.A. Houlberg, and S.L. Milora, Vac Sci. Technol. 20, 1254 (1982).

[51] H. Lomax and H. E. Bailey, A Critical Analysis of Various Numerical Integration Methods for Computing the flow of a Gas in Chemical Nonequilibrium, NASA TN.D. $4109,1967$.

[52] C. E. Treanor, Math. Computation, 20, (1966) 39.

[53] E. S. Oran and J. P. Boris, Numerical Simulation of Reactive Flow, (Elsevier Science, New York, 1987).

[54] K.L. Bell, et. al., J. Phys. Chem. Ref. Data, 12, 1983.

[55] P. B. Parks, Private Communication, October 1990.

[56] L.L. Vahala, A.G. Elcashlan, G.A. Gerdin, and P. Parks, Rev. Sci. Instrum., 61(10), 3140 (1990).

[57] D.A. Anderson, J.C. Tannehill, and R.H. Pletcher, Computational Fluid Mechanics and Heat Transfer, (McGraw Hill, New York, 1984).

[58] J.D. Anderson, Jr., Modern Compressible Flow, (McGraw Hill, New York, 1990).

[59] D.M. Thomas, R.K. Fisher, J.S. Leffler, P.B. Parks, S.C. McCool, W.L. Rowan, F.D. Anderson, and M.L. Walker, Bult. Amer. Phys. Soc. 33, 1966 (1988).

[60] S.C. McCool, F.C. Anderson, M.L. Walker, R.V. Bravenec, D.L. Brower, R.K. Fisher, K.W. Gentle, J.S. Leffler, N.C. Luhmann, Jr., W.A. Peebles, P.E. Phillips, B. Riehards, W.L. Rowan, P.M. Schoch, B.A. Smith, K.W. Wenzel, A.J. Wooton, Bult. Amer. Phys. Soc. $\underline{33}, 2024$ (1988).

[61] Chul Park, On Convergence of Computation of Chemically Reacting Flows, AIAA Paper No. 85-0247, 1985.

[62] F.J. Moody, Introduction To Unsteady Thermofluid Mechanics, (John Wiley, New York, 1990).

[63] R.K. Fisher, J.M. McChesney, P.B. Parks, J.J. Terry, E.S. Marmar, and D.W. Johnson, Bult. Amer. Phys. Soc. $\underline{35}$ (2091) 1990. 
[64] R.K. Fisher, J.M. McChesney, P.B. Parks, D.M. Thomas, S.C. McCool, and W.L. Rowan, Rev. Sci. Instrum., 61(10), 3196 (1990).

[65] R.K. Fisher, J.S. Leffler, A.M. Howald, and P.B. Parks, Fusion Technol. 13, (1988) 536. 


\section{APPENDIX A}

The ionization fraction $f_{j}$ is a function of the ablatant cloud temperature and electron density. Under steady state conditions $f_{j}$ is given by equation (2-4) which is

$$
f_{j}=\left(\prod_{m=0}^{j-1} R_{m, m+1}\right) /\left(1+\sum_{l=1}^{Z} \prod_{m=0}^{l-1} R_{m, m+1}\right)=\prod_{m=0}^{j-1} R_{m, m+1} / D
$$

where

$$
D=1+\sum_{l=1}^{Z} \prod_{m=0}^{l-1} R_{m, m+1}
$$

and $R_{m, m+1}$ is the ratio between the collisional ionization rate, and the sum of the collisional, radiative, and dielectronic recombination rates, i.e.,

$$
R_{m, m+1}=\frac{C_{m}^{c o l l}}{\alpha_{m+1}^{\text {coll }}+\alpha_{m+1}^{\text {rod }}+\alpha_{m+1}^{\text {diel }}} \equiv \frac{C_{m}^{c}}{\alpha_{m+1}^{c}+\alpha_{m+1}^{r}+\alpha_{m+1}^{d}}
$$

where $C_{m}^{c}$ is the collisional ionization rate from state $m$ to $m+1$ and $\alpha_{m+1}^{c}, \alpha_{m+1}^{r}$, and $\alpha_{m+1}^{d}$ are the collisional, radiative and dielectronic recombination rates from state $m+1$ to $\mathrm{m}$ respectively.

Inspection of eq. (2-4) shows that the degree of ionization, $f_{j}$, is a function of the local ablation cloud temperature and electron density, hence the spatial derivatives of $f_{j}$ is then 
written as

$$
\frac{d f_{j}}{d r}=\sum_{m=0}^{z-1} \frac{d f_{j}}{d R_{m, m+1}}\left[\frac{d R_{m, m+1}}{d \theta} \frac{d \theta}{d r}+\frac{d R_{m, m+1}}{d n_{e L}} \frac{d n_{e L}}{d r}\right]
$$

where $\mathrm{n}_{\mathrm{eL}}$ is the ablatant electron number density and is given by

$$
n_{a L}=n \sum_{j=1}^{z} i f_{j}
$$

thus,

$$
\frac{d n_{a l}}{d r}=\sum_{j=1}^{z} i_{j} \cdot \frac{d n}{d r}+n \sum_{j=1}^{z} j \frac{d f_{j}}{d r}
$$

Inserting equation (A5) into equation (A3) results

$$
\begin{gathered}
\frac{d f_{j}}{d r}=\sum_{m=0}^{z-1} \frac{d f_{j}}{d R_{m, m+1}} \frac{d R_{m, n+1}}{d \theta} \frac{d \theta}{d r}+\sum_{m=0}^{z-1} \frac{d f_{j}}{d R_{m, m+1}} n \sum_{j=1}^{z} i f_{j} \\
\cdot \frac{1}{n} \frac{d n}{d r}+\sum_{m=0}^{z-1} \frac{d f_{j}}{d R_{m, m+1}} \frac{d R_{m, n+1}}{d n_{a L}} n \cdot \sum_{j=1}^{z} j \frac{d f_{j}}{d r} \\
\frac{d f_{j}}{d r}=A_{j} \frac{d \theta}{d r}+B_{j} \frac{1}{n} \frac{d n}{d r}+C_{j} \sum_{j=1}^{z} \frac{d f_{j}}{d r}
\end{gathered}
$$

in which we defined

$$
A_{j} \equiv \sum_{m=0}^{z-1} \frac{d f_{j}}{d R_{m, m+1}} \frac{d R_{m, m+1}}{d \theta}
$$

To evaluate the expressions $A_{j}, B_{j}$, and $C_{j}$ we utilize equation (A1) to obtain, 


$$
\begin{gathered}
B_{j} \equiv \sum_{m=0}^{z-1} \frac{d f_{j}}{d R_{m, m+1}} \frac{d R_{m, m+1}}{d n_{a L}} n \sum_{j+1}^{Z} i f_{j} \\
C_{j} \equiv B_{j} / \sum_{j=1}^{Z} \dot{I}_{j} \\
\frac{d f_{j}}{d R_{m, m+1}}=\frac{1}{D^{2}}\left[D \cdot \frac{d}{d R_{j-1 j}}\left(\prod_{m=1}^{t-1} R_{m, m+1}\right)-\left(\prod_{m=0}^{j-1} R_{m, m+1}\right) \cdot \frac{d r}{d R_{j-1 j}}\right]
\end{gathered}
$$

and equation (A2) to get $\frac{d R_{m, m+1}}{d \theta}$ as follows:

$$
\frac{d R_{m,+1}}{d \theta}=\frac{d R_{m,+1}}{d C_{m}^{c}} \frac{d C_{m}^{c}}{d \theta}+\frac{d R_{m,+1}}{d \alpha_{m+1}^{c \alpha}} \frac{d \alpha_{m+1}^{\infty \alpha r}}{d \theta}
$$

where

$$
\alpha_{m+1}^{\infty \alpha}=\alpha_{m+1}^{c}+\alpha_{m+1}^{d}+\alpha_{m+1}^{r}
$$

using the expressions given in chapter 2 for ionization and recombination rates we obtain:

$$
\begin{gathered}
\frac{d C_{m}^{c}}{d \theta}=\frac{C_{m}^{c}}{\theta}\left[\frac{1}{2}+x_{j}\left(\frac{1-2 e^{-x_{j}}}{1-e^{-x_{j}}}\right)\right] \\
\frac{d \alpha_{m+1}^{c}}{d \theta}=\frac{\alpha_{m+1}^{c}}{\theta}\left[-1-x_{j} \frac{e^{-x_{j}}}{1-e^{-x_{j}}}\right] \\
\frac{d \alpha_{m+1}^{r}}{d \theta}=\frac{\alpha_{m+1}^{r}}{\theta}\left[-\frac{3}{2}-x_{j}+\frac{e^{-x_{j}}}{E_{1}\left(x_{j}\right)}\right]
\end{gathered}
$$

To evaluate the remaining terms in equation (A11), we use equation (A1) to get 


$$
\begin{gathered}
\frac{d \alpha_{m+1}^{d}}{d \theta}=\frac{\alpha_{m+1}^{d}}{\theta}\left[-\frac{3}{2}+\frac{100}{7\left(N_{t}+200\right)}+\frac{E n i(j)}{T}\right] \\
\frac{d R_{m+1+1}}{d C_{m}^{c}}=\frac{1}{\alpha_{m+1}^{10 x}}
\end{gathered}
$$

and

$$
\frac{d R_{m, m+1}}{d \alpha_{m+1}^{\infty \alpha x}}=-\frac{R_{m, m+1}}{\alpha_{m+1}^{\infty \alpha x}}
$$

Inspection of the ionization and recombination rates shows that only $\alpha^{c}$ and $\alpha^{d}$ are function of the electron density $n_{e L}$, hence using equation (A-2) we have,

$$
\frac{d R_{m, m+1}}{d n_{a L}}=\frac{d R_{m,+1}}{d \alpha_{m+1}^{c}} \frac{d \alpha_{m+1}}{d n_{a L}}+\frac{d R_{m, m+1}}{d \alpha_{m+1}^{d}} \frac{d \alpha_{m+1}^{d}}{d n_{a L}}
$$

Again using $\alpha^{c}$ and $\alpha^{r}$ expression given in chapter 2 we get

$$
\frac{d \alpha_{m+1}^{c}}{d n_{a L}}=\frac{\alpha_{m+1}^{c}}{n_{a L}}
$$

and

$$
\frac{d \alpha_{m+1}^{d}}{d n_{a L}}=-\frac{\alpha_{m+1}^{d}}{n_{a L}} \frac{200}{7\left(N_{t}+200\right)}
$$

Up to this point we utilize equations (A12 - A-17) to evaluate equation (A11) and use equations (A19 - A20) to evaluate equation (A18). The resulting equations and equation (A10) give the expressions $A_{j}, B_{j}$, and $C_{j}$. Knowing these expressions we return to 
equation (A6) in which we insert equation (3-15) for $d \theta / d r$ to get:

$$
\begin{aligned}
\frac{d f_{j}}{d r} & =\frac{A_{j}}{L}\left[S-\left(\frac{\gamma-1}{d}\right) \frac{d W}{d r}-A_{*} \frac{d}{d r} \sum_{j=1}^{z} Q f_{j}-\frac{\theta}{L_{*}} \sum_{j=1}^{Z} j \frac{d f_{j}}{d r}\right]- \\
& B_{j}\left[\frac{2}{r}+\frac{1}{2 W} \frac{d W}{d r}\right]+C_{j} \sum j \frac{d f}{d r} \\
& \equiv D_{l}-E_{j} \sum_{j} Q_{j} \frac{d f_{j}}{d r}+H_{j} \sum j \frac{d f_{j}}{d r}
\end{aligned}
$$

in which we have dropped the primes, and used conservation of mass, $n \backslash W r^{2}=1$, to obtain,

$$
-\frac{1}{n} \frac{d n}{d r}=\frac{2}{r}+\frac{1}{d W} \frac{d W}{d r}
$$

The new variables $D_{j}, E_{j}$, and $H_{j}$ are defined as follows:

$$
\begin{gathered}
D_{j}=\frac{S}{L} A_{j}-\frac{2}{r} B_{j}-\left(\frac{(\gamma-1)}{2 L} A_{j}+\frac{1}{2 W} B_{j}\right) \frac{d W}{d r} \\
E_{j}=\frac{A_{*} A_{j}}{L} \\
H_{j}=C_{j}-\frac{\theta}{L_{*}} \frac{A_{j}}{L}
\end{gathered}
$$

Multiplying both sides of equation (A21) by $Q_{j}$ and $j$ and summing over $j$ respectively, one has after some rearrangement,

$$
\sum_{j=1}^{Z} Q_{j} \frac{d f_{j}}{d r}\left(1+\sum_{j} Q_{j} E_{j}\right)=\sum_{j=1}^{z} Q_{j} D_{j}+\sum_{j} Q_{j} H_{j} \sum_{j} j \frac{d f_{j}}{d r}
$$

and 


$$
\sum j \frac{d f_{j}}{d r}\left(1-\sum_{j} j H_{j}\right)=\sum_{j} j D_{j}-\sum_{j} j E_{j} \sum Q_{j} \frac{d f_{j}}{d r}
$$

Using equations (A25) and (A26) we solve for the terms

$$
\sum_{j=1}^{Z} Q_{j} \frac{d f_{j}}{d r} \text { and } \sum_{j=1}^{z} j \frac{d f_{j}}{d r}
$$

to obtain

$$
\sum_{j} Q_{j} \frac{d f_{j}}{d r}=\frac{1}{\Delta}\left[\sum_{j} Q_{j} H_{j} \sum_{j} D_{j}+\sum_{j} Q_{j} D_{j}\left(1-\sum_{j} H_{j}\right)\right]
$$

and

$$
\sum j \frac{d f_{j}}{d r}=\frac{1}{\Delta}\left[\left(1+\sum Q_{j} E_{j}\right) \sum D_{j}-\sum j E_{j} \sum Q_{j} D_{j}\right]
$$

where

$$
\Delta=\sum_{j} Q_{j} H_{j} \sum_{j} j E_{j}+\left(1+\sum Q_{j} E_{j}\right)\left(1-\sum_{j} H_{j}\right)
$$

Rewrite equation (A22) as

$$
D_{j}=V_{j}-W_{j} \frac{d W}{d r}
$$

where 


$$
\begin{gathered}
V_{J} \equiv \frac{S A_{J}}{L}-\frac{2}{r} B_{J} \\
W_{J} \equiv \frac{(\gamma-1)}{2 L} A_{J}+\frac{1}{2 W} B_{J}
\end{gathered}
$$

Multiplying equation (A30) by $Q_{j}$ and $j$ and summing over $j$ gives respectively;

$$
\sum_{j} Q_{j} D_{j}=\sum_{j} Q_{j} V_{j}-\frac{d W}{d r} \sum_{j} Q_{j} W_{j}
$$

and

$$
\sum j D_{j}=\sum_{j} V_{j}-\frac{d W}{d r} \sum j W_{\jmath}
$$

Finally, substituting equations (A33) and (A34) into equations (A27) and (A28) we have

$$
\sum_{j=1}^{2} Q_{j} \frac{d f_{j}}{d r}=U_{1}-U_{2} \frac{d W}{d r}
$$

and

$$
\sum_{j=1}^{Z} j \frac{d f_{j}}{d r}=U_{3}-U_{4} \frac{d W}{d r}
$$

where 


$$
\begin{gathered}
U_{1}=\frac{1}{\Delta}\left[\sum Q_{j} H_{j} \sum j V_{j}+\left(1-\sum j H_{j}\right) \sum Q_{j} V_{j}\right] \\
U_{2}=\frac{1}{\Delta}\left[\sum Q_{j} H_{j} \sum j W_{j}+\left(1-\sum j H_{j}\right) \sum Q_{j} W_{j}\right] \\
U_{3}=\frac{1}{\Delta}\left[\sum j V_{j}\left(1+\sum Q_{j} E_{j}\right)-\sum j E_{j} \sum Q_{j} V_{j}\right] \\
U_{4}=\frac{1}{\Delta}\left[\sum j W_{j}\left(1+\sum Q_{j} E_{j}\right)-\sum j E_{j} \sum Q_{j} W_{j}\right]
\end{gathered}
$$




\section{APPENDIX B}

\section{Derivation of the heat flux degradation, $\frac{\mathrm{dg}}{\mathrm{dr}}$ for carbon ablatant}

The energy distribution far from the pellet, $\mathrm{f}_{\infty}(\mathrm{E})$, is assumed to be Maxwellian at the background plasma electron temperature:

$$
f_{M}(E)=\frac{n_{e}}{2}\left(\pi T_{e}\right)^{-3 / 2} E^{1 / 2} \exp \left(-E / T_{2}\right)
$$

Parks et.al. obtained the distribution function for plasma electrons, by solving Boltzmann equation, which is given by [6]

$$
f_{e}(E, r)=f_{M}(\Lambda)\left(\frac{E}{\Lambda}\right)^{\left(p+\kappa Z-\frac{1}{2}\right)}
$$

The expression $\Lambda$ is given by [6]

$$
\Lambda=E\left[1+\frac{9.7610^{-14} M \tau}{E^{(p+1)}}\right]^{(p+1)^{-1}}
$$

where $M$ is the atomic mass of the pellet material in atomic mass units $\sim 2 Z, E, T_{e}$ are the electron volts, and $p=0.72$. The ablation cloud thickness, $\tau=\int_{r_{0}}^{\infty} n(r) d r$, has units of inverse centimeters squared and $r_{0}$ is the pellet radius. The parameter $K$ is given by [6]:

$$
\kappa=\ln \Lambda_{0}(E) / \ln (E / I)
$$

where $\Lambda_{0}(E)=0.591 \mathrm{a}_{0} / S Z^{1 / 3} \lambda$ and the other definitions are [6] 


$$
\begin{gathered}
a_{0}=\frac{h^{2}}{m_{e} e^{2}} \text { (Bohr radius), } \\
\lambda=\frac{h}{m_{e} v} \text { (de Broglie wavelength), } \\
\mathrm{I}=5.5 \mathrm{Z} \text { and } \mathrm{S}=\mathbf{0 . 5 8 .}
\end{gathered}
$$

If we divide both sides of equation (B3) by $T_{c}$ then we have:

$$
\frac{\Lambda}{T_{e}}=\left[t^{1 / \beta}+u\right]^{\beta}
$$

in which we have defined

$$
\begin{gathered}
u=\frac{9.7610^{-14} M \tau}{T^{(p+1)}} \\
t=E / T \\
\beta=1 /(p+1)
\end{gathered}
$$

Similarly, equation (B2) can be written as

$$
f_{e}(E, r)=f_{M}(\Lambda) \frac{\left(\frac{E}{T_{e}}\right)^{\left(p+\nless Z-\frac{1}{2}\right)}}{\left(\frac{\Lambda}{T_{e}}\right)^{\left(p+\ll Z-\frac{1}{2}\right)}}
$$

then, substituting equations (B1) and (B4) into (B5) gives 


$$
f_{e}(E, r)=\frac{n_{e}}{2 T_{e} \pi^{3 / 2}} \frac{t^{\left(p+\alpha z-\frac{1}{2}\right)}}{\left[t^{1 / \beta}+u\right]^{K}} \exp \left[-\left(t^{1 / \beta}+u\right)^{\beta}\right]
$$

in which we defined $K=\beta(p-1+K Z)$.

Inspection of equation (B6) shows that the electron distribution function becomes Maxwellian when $\mathrm{u}$ tends to zero as expected.

The energy flux at the pellet surface, $q_{0}$, is given by [6]

$$
q_{0}=2 \pi \int_{0}^{1} \mu d u \int_{0}^{\infty} v E f_{e}(E, r) d E
$$

Substituting equation (B6) into equation (B7) yields the attenuation coefficient, $\eta=q_{d} / q_{\infty}$, defined in reference [6], i.e.,

$$
\eta=\frac{1}{2} \int_{0}^{\infty} t^{(2+K / \beta)}\left(t^{1 / \beta}+u\right)^{-K} \exp \left[-\left(t^{1 / \beta}+u\right)^{\beta}\right] d t
$$

For a given pellet radius, $r_{0}$, ambient plasma temperature and density the heat flux $q(r)$ is only function of the dimensionless ablation cloud thickness $u$. Hence equation (B8) can be written as

$$
q(u) \equiv \frac{q_{-}}{2} \int_{0}^{\infty} G^{\prime}(u) d t
$$

where $G^{\prime}(u)$ is the integrand in equation (B8). 
Using equations (B8) and (B9) an expression for $\mathrm{dq}(\mathrm{u}) / \mathrm{du}$ is obtained as follows

$$
\frac{d q}{d u}=\frac{1}{2} \int_{0}^{\infty} \frac{\dot{G}(u)}{\left(t^{1 / \beta}+u\right)}\left[K+\beta\left(t^{1 / \beta}+u\right)^{\beta}\right] d t \equiv G(u)
$$

Hence,

$$
\frac{d q(r)}{d r}=\frac{d q(u)}{d u} \cdot \frac{d u}{d \tau} \cdot \frac{d \tau}{d r}=G(u) \frac{9.7610^{-14} M n_{*} r_{*}}{T_{*}^{(p+1)}} \frac{1}{r^{2} \sqrt{W}}
$$

in which we have used

$$
\begin{aligned}
& \frac{d \tau}{d r}=-n(r) \\
& \frac{d u}{d \tau}=\frac{9.7610^{-14} M}{T_{e}^{(p+1)}}
\end{aligned}
$$

and the normalized equation form mass conservation, $\mathrm{n}^{\prime} \mathrm{VWr} \mathbf{r}^{2}=1$.

To eliminate the time consuming process of integrating the expression $G(u)$, equation (B10), at each integration step in the code, it was decided to evaluate this expression as a function of the ablation cloud thickness, $u$ (for ten energy groups) and fit a polynomial to the resulting curve. This polynomial, of course, belongs to a given pellet radius, ambient plasma temperature and density. 


\section{APPENDIX C}

\section{Derivation of ionization Rate $S_{j}^{\mathbf{X}}$}

The ionization rate coefficient, $S_{j}^{x}$, introduced in chapter 5 is determined as consistent with ref. 6 , i.e..

$$
S_{f}^{x}\left(T_{e}, n_{e}, r\right)=2 \pi \int_{0}^{1} \mu d \mu \int_{\phi}^{\infty} \sigma^{f}(E) v_{e} f_{e}(E, r) d E
$$

Where $\sigma^{j}(E)$ represents the ionization cross-section for electrons of energy $E$, and is given by [54]

$$
\sigma_{(E)}^{\prime}=\frac{1}{\phi, E}\left\{A^{J} \ln \left(\frac{E}{\phi_{j}}\right)+\sum_{j=1}^{N} B_{i}^{J}\left(1-\frac{\phi_{j}}{E}\right)^{\prime}\right\}
$$

The parameters $\phi_{j}, A^{j}$ and $B_{i}^{j}$, are given in table 1. Substituting equation (B6) into equation (C1) yields;

$$
S_{j}^{x}=\frac{v_{e}}{4} \int_{\phi / T_{e}}^{-} \sigma_{(\mathcal{B})}^{\alpha} \frac{t^{(p+K Z)}}{\left[t^{(p+1)}+u\right]^{K}} \cdot \exp \left[-\left(t^{1 / \beta}+u\right)^{\beta}\right]
$$

Equation (C3) is evaluated, for a given plasma conditions, as a function of the cloud thickness, $u$, and a polynomial is fitted to the resulting curve. 
TABLE 1.

The parameters $\Phi_{j}, A^{j}$, and $B_{i}^{j} . A$ and $B$ are in units of $10^{-13} \mathrm{ev}^{2}-\mathrm{cm}^{2}[54]$

\begin{tabular}{c|c|c|c|c|c|c}
\hline Species & $\Phi_{j}(\mathrm{ev})$ & $\mathrm{A}^{\mathrm{j}}$ & $\mathrm{B}_{1}{ }^{\mathrm{j}}$ & $\mathrm{B}_{2}{ }^{\mathrm{j}}$ & $\mathrm{B}_{3}{ }^{\mathrm{j}}$ & $\mathrm{B}_{4}{ }^{\mathrm{j}}$ \\
\hline $\mathrm{CI}$ & 11.26 & 2.1143 & -1.9647 & -.6084 & & \\
\hline $\mathrm{CII}$ & 24.38 & 1.0824 & -0.1611 & -.8563 & 0.9062 & \\
\hline $\mathrm{CIII}$ & 47.89 & 0.7150 & -0.0410 & 0.1754 & & \\
\hline $\mathrm{CIV}$ & 64.49 & 0.4667 & -0.1298 & 0.2577 & 0.9561 & 0.644 \\
\hline $\mathrm{CV}$ & 392.08 & 0.7960 & -.5004 & 0.8836 & & \\
\hline $\mathrm{CVI}$ & 489.98 & 0.4000 & & & & \\
\hline
\end{tabular}




\section{APPENDIX D}

The derivations of the expressions $U_{1}^{x}, U_{2}{ }^{x}, U_{3}^{x}$, and $U_{4}^{x}$, used in the plasma electrons model, are performed using the same procedure as in Appendix A. The major difference, which show the plasma electrons effect, is in the ratio $R_{m, m+1}$ which is given by equation (5-11) as

$$
\begin{aligned}
R_{m m+1} & =\frac{C_{m}^{\text {coll }}+C_{m}^{x}}{\alpha_{m+1}^{\text {coll }}+\alpha_{m+1}^{\text {rad }}+\alpha_{m+1}^{\text {dlel }}} \\
& \equiv \frac{C_{m}^{c}+C_{m}^{x}}{\alpha_{m+1}^{c}+\alpha_{m+1}^{r}+\alpha_{m+1}^{d}}
\end{aligned}
$$

We should note that the same notations in Appendix A are used here but their values are different from those in Appendix A. Therefore, the final results are different and have different notations. The ionization fraction $f_{j}$ as given by equation (5-10) is

$$
f_{j}=\left(\prod_{m=0}^{l-1} R_{m, m+1}\right) /\left(1+\sum_{l=0}^{Z} \prod_{m=0}^{l-1} R_{m, m+1}\right)=\left(\prod_{m=0}^{j-1} R_{m, m+1}\right) / D
$$

The spatial derivative of $f_{j}$ is given by:

$$
\frac{d f_{j}}{d r}=\sum_{m=0}^{z-1} \frac{d f_{J}}{d R_{m, m+1}}\left[\frac{d R_{m, m+1}}{d \theta} \frac{d \theta}{d r}+\frac{d R_{m, m+1}}{d n_{e L}} \frac{d n_{e l}}{d r}+\frac{d R_{m, m+1}}{d u} \frac{d u}{d r}\right]
$$


Inspection of equation (D3) shows the dependence of $f_{j}$ on the ablation cloud thickness, u, which reflects the plasma electrons effects and it indicate the departure from equation (A3).

The ablatant electron number density, $\mathrm{n}_{\mathrm{el}}$, is given by

$$
n_{e l}=\sum_{j=1}^{Z} i f
$$

Thus,

$$
\frac{d n_{a l}}{d r}=\sum_{j=1}^{Z} i f_{j} \cdot \frac{d n}{d r}+n \sum_{j=1}^{Z} j \frac{d f_{j}}{d r}
$$

Inserting this equation into equation (D3) gives,

$$
\frac{d f_{i}}{d r}=A_{j} \frac{d \theta}{d r}+B_{j} \frac{1}{n} \frac{d n}{d r}+C_{j} \sum j \frac{d f_{j}}{d r}+Y_{j}
$$

where $A_{j}, B_{j}$, and $C_{j}$ are similar to those defined in Appendix $A$ by equations (A7 - A9). The new term $Y_{j}$ is given by

$$
Y_{j}=\sum_{m=0}^{z-1} \frac{d f_{j}}{d R_{m, m+1}} \frac{d u}{d r}
$$

The quantities $\tau$ and $u$ are introduced in Appendix B, Hence,

$$
\frac{d u}{d r}=\frac{-9.76}{T_{c}^{1.72}} \frac{10^{-14} M}{r_{*}^{2} \sqrt{W}}
$$


The change in $R_{\mathrm{m}, m+1}$ with respect to $u$ is, from equation (D1)

$$
\frac{d R_{m, m+1}}{d u}=\frac{1}{\alpha_{m+1}^{20 x}} \frac{d C_{m}^{x}}{d u}
$$

The rate coefficients introduced in chapter 5 and derived in Appendix $C$ is given by

$$
S^{x}=\frac{\nu_{e}}{4} \int_{\phi / T_{e}}^{\infty} \sigma_{(E)} \frac{t^{(p+\kappa 2)}}{\left[t^{(p+1)}+u\right]^{K}} \exp \left[-\left(t^{1 / \beta}+u\right)^{\beta}\right] d t
$$

Since

$$
C_{m}^{x} \equiv \frac{n_{e}}{n_{e l}} S_{m}^{x}
$$

then,

$$
\frac{d C_{m}^{x}}{d u}=\frac{n_{e}}{n_{e L}} d S_{m}^{x} / d u
$$

To evaluate equation (D9) we define $\mathrm{S}^{\mathrm{x}}$ given by equation (D8) as

$$
S^{x}=\frac{v_{e}}{4} \int_{\phi T T_{e}}^{\infty} F(u) d t
$$

then we may write finally

$$
\frac{d S^{x}}{d u}=-\frac{\nu_{e}}{4} \int_{\phi T_{e}}^{\infty} \frac{F(u)}{\left[t^{(1+p)}+u\right]}\left[K+\beta\left(t^{(p+1)}+u\right)^{\beta}\right] d t
$$


In a completely analogous way, the expressions $\frac{\mathrm{dS}}{}$ is fitted by a polynomial curve using least squares. $\mathrm{du}$

At this point the term $Y_{j}$ can be evaluated by substituting equations (D6, D7, D9, D11, A10) into equation (D5). Moreover, we should note that the derivatives $\frac{d f}{d R}, \frac{d R_{m, m+1}}{d \theta}$, and $\underline{d R}_{m, m+1}$ are written by direct analogy with those given in Appendix $\mathrm{dR}_{\mathrm{m}, \mathrm{m}+1} \frac{\mathrm{dR}}{\mathrm{d} \theta} \mathrm{dn}_{\mathrm{eL}}$

B. The latter, however, has an extra term due to the dependence of $C_{m}{ }^{x}$ on $n_{e L}$, namely

$$
\frac{d R_{m, m+1}}{d n_{e L}}=\frac{d R_{m, m+1}}{d C_{m}^{x}} \frac{d C_{m}^{x}}{d n_{a L}}+\frac{d R_{m, m+1}}{d \alpha_{m+1}^{c}} \frac{d \alpha_{m+1}^{c}}{d n_{e L}}+\frac{d R_{m, m+1}}{d \alpha_{m+1}^{d}} \frac{d \alpha_{m+1}^{d}}{d n_{e L}}
$$

where

$$
\begin{gathered}
\frac{d R_{m+1}}{d C_{m}^{x}}=\frac{1}{\alpha_{m+1}^{\infty x x}} \\
\frac{d C_{m}^{x}}{d n_{a L}}=-\frac{C_{m}^{x}}{n_{e L}}
\end{gathered}
$$

We may recall now equation (D4) for $\frac{\mathrm{df}}{\mathrm{dr}}$,

$$
\frac{d f_{j}}{d r}=A_{j} \frac{d \theta}{d r}+B_{j} \frac{1}{n} \frac{d n}{d r}+C_{j} \sum j \frac{d f_{j}}{d r}+Y_{j}
$$

then after elimination of $\frac{d \theta}{d r}$ and $\frac{1}{n} \frac{d n}{d r}$ and further manipulation, we have, 


$$
\frac{d f_{j}}{d r}=D_{j}-E_{j} \sum_{j} Q_{j} \frac{d f_{j}}{d r}+H_{j} \sum j \frac{d f_{j}}{d r}
$$

where:

$$
\begin{gathered}
E_{j}=\frac{A_{*} A_{j}}{L} \\
H_{j}=C_{j}-\frac{\theta}{L_{*}} \frac{A_{j}}{L} \\
D_{J} \equiv \frac{S}{L} A_{j}-\frac{2}{r} B_{j}+Y_{j}-\frac{d W}{d r}\left[\frac{(\gamma-1)}{2 L} A_{j}+\frac{1}{2 W} B_{j}\right] \equiv V_{j}-W_{j} \frac{d W}{d r} \text { (D) }
\end{gathered}
$$

Finally by direct analogy with equations (A35) and (A36) we write

$$
\begin{aligned}
& \sum_{j=1}^{Z} Q_{j} \frac{d f_{j}}{d r}=U_{1}^{x}-U_{2}^{x} \frac{d W}{d r} \\
& \sum_{j=1}^{Z} j \frac{d f_{j}}{d r}=U_{3}^{x}-U_{4}^{x} \frac{d W}{d r}
\end{aligned}
$$

where

$$
\begin{aligned}
& U_{1}^{x}=\frac{1}{\Delta^{x}}\left[\sum_{j} Q_{j} H_{j} \sum_{j} j V_{j}+\left(1-\sum_{j} j H_{j}\right) \sum_{j} Q_{j} V_{j}\right] \\
& U_{3}^{x}=\frac{1}{\Delta^{x}}\left[\sum_{j} j U_{j}\left(1+\sum_{j} Q_{j} E_{j}\right)-\sum_{j} j E_{j} \sum_{j} Q_{j} V_{j}\right]
\end{aligned}
$$




$$
\begin{aligned}
& U_{2}^{x}=\frac{1}{\Delta^{x}}\left[\sum_{j} Q_{j} H_{j} \sum_{j} j W_{j}+\left(1-\sum_{j} j H_{j}\right) \sum_{j} Q_{j} W_{j}\right] \\
& U_{4}^{x}=\frac{1}{\Delta^{x}}\left[\sum_{j} j W_{j}\left(1+\sum_{j} A_{j} E_{j}\right)-\sum_{j} j E_{j} \sum_{j} Q_{j} W_{j}\right]
\end{aligned}
$$

in which

$$
\Delta^{x}=\sum_{j} Q_{j} H_{j} \sum_{j} j E_{j}+\left(1+\sum Q_{j} E_{j}\right)\left(1-\sum j H_{j}\right)
$$




\section{APPENDIX E}

\section{Shock Equations}

Let subscripts 1 and 2 refer to conditions just upstream and downstream of the shock. It is assumed that the atomic processes are frozen across the shock, i.e., $L_{i}$ is unchanged. The conservation laws of mass, momentum, and energy across the shock are:

$$
\begin{gathered}
\rho_{1} v_{1}=\rho_{2} v_{2}=\frac{\dot{m}}{A} \\
P_{1}-P_{2}=\frac{\dot{m}}{A}\left(v_{2}-v_{1}\right) \\
T_{1}\left[1+\left(\frac{\gamma-1}{2}\right) M_{1}^{2}\right]=T_{2}\left[1+\left(\frac{\gamma-1}{2}\right) M_{2}^{2}\right]
\end{gathered}
$$

Equation (E3) can be written as:

$$
\frac{T_{2}}{T_{1}}=\frac{1+\left(\frac{\gamma-1}{2}\right) M_{1}^{2}}{1+\left(\frac{\gamma-1}{2}\right) M_{2}^{2}}
$$

From equation (E1) and the perfect gas law, the ration of temperature can also be expressed as:

$$
\frac{T_{2}}{T_{1}}=\frac{P_{2}}{P_{1}} \frac{n_{1}}{n_{2}}=\frac{P_{2}}{P_{1}} \frac{v_{2}}{v_{1}}
$$

But since 


$$
v_{1}^{2}=\frac{\gamma T_{t} L_{t}}{m} M_{1}^{2}
$$

and

$$
v_{2}^{2}=\frac{\gamma T_{2} L_{t}}{m} M_{2}^{2}
$$

the velocity ratio is:

$$
\frac{v_{2}}{v_{1}}=\frac{M_{2}}{M_{1}} \sqrt{\frac{T_{2}}{T_{1}}}
$$

Therefore the temperature ratio in equation (E5) becomes:

$$
\frac{T_{2}}{T_{1}}=\frac{M_{2}}{M_{1}} \frac{P_{2}}{P_{1}} \sqrt{\frac{T_{2}}{T_{1}}}
$$

So that:

$$
\frac{T_{2}}{T_{1}}=\left(\frac{P_{2}}{P_{1}}\right)^{2}\left(\frac{M_{2}}{M_{1}}\right)^{2}
$$

By combining equations (E4) and (E7), the temperature ratio can be eliminated so that the pressure ratio across the shock is:

$$
\frac{P_{2}}{P_{1}}=\frac{M_{1}}{M_{2}} \sqrt{\frac{1+\left(\frac{\gamma-1}{2}\right) M_{1}^{2}}{1+\left(\frac{\gamma-1}{2}\right) M_{2}^{2}}}
$$


The density ratio across the shock can be written as:

$$
\frac{\rho_{2}}{\rho_{1}}=\frac{P_{2}}{P_{1}} \frac{T_{1}}{T_{2}}
$$

By combining equations (E4) and (E8), the density ratio becomes:

$$
\frac{\rho_{2}}{\rho_{1}}=\frac{M_{1}}{M_{2}} \sqrt{\frac{1+\left(\frac{\gamma-1}{2}\right) M_{2}^{2}}{1+\left(\frac{\gamma-1}{2}\right) M_{1}^{2}}}
$$

The relationship between $M_{1}$ and $M_{2}$ will now be examined.

Since $M^{2}=\frac{m v^{2}}{V T L i}$ and $P=\underset{m}{\rho} T L_{i}$, then the velocity and the Mach number are related as:

$$
\rho v^{2}=\gamma P M^{2}
$$

Accordingly, the momentum equation (E2) becomes:

$$
P_{1}+\gamma P_{1} M_{1}^{2}=P_{2}+\gamma P_{2} M_{2}^{2}
$$

or

$$
\frac{P_{2}}{P_{1}}=\frac{1+\gamma M_{1}^{2}}{1+\gamma M_{2}^{2}}
$$

By combining equations (E8) and (E10), the following is obtained: 


$$
\frac{M_{1} \sqrt{1+\left(\frac{\gamma-1}{2}\right) M_{1}^{2}}}{1+\gamma M_{1}^{2}}=\frac{M_{2} \sqrt{1+\left(\frac{\gamma-1}{2}\right) M_{2}^{2}}}{1+\gamma M_{2}^{2}}
$$

from which,

$$
M_{2}=\sqrt{\frac{2+(\gamma-1) M_{1}^{2}}{2 \gamma M_{1}^{2}-(\gamma-1)}}
$$

Temperature, pressure, and density ratios can now be expressed as a functions of only a single Mach number:

$$
\begin{gathered}
\frac{T_{2}}{T_{1}=} \frac{\left[2 \gamma M_{1}^{2}-(\gamma-1)\right]\left[2+(\gamma-1) M_{1}^{2}\right]}{(\gamma+1)^{2} M_{1}^{2}} \\
\frac{P_{2}}{P_{1}}=\frac{2 \gamma M_{1}^{2}-(\gamma-1)}{\gamma+1} \\
\frac{n_{2}}{n_{1}}=\frac{v_{1}}{v_{2}}=\frac{(\gamma+1) M_{1}^{2}}{2+(\gamma-1) M_{1}^{2}}
\end{gathered}
$$

For the equilibrium channel flow model, it is assumed that the ablatant is in instantaneous charge-state equilibrium on both sides of the discontinuity. With this in mind the conservation laws of energy across the shock is:

$$
\frac{\gamma T_{2} L_{2}}{(\gamma-1) m}+\frac{V_{2}^{2}}{2}+\left.\frac{1}{m} \sum_{j} Q_{j} f_{j}\right|_{2}=\frac{\gamma T_{1} L_{1}}{(\gamma-1) m}+\frac{V_{1}^{2}}{2}+\frac{1}{m} \sum_{j} Q_{j} f_{j}
$$

or 


$$
\frac{\gamma T_{2} L_{2}}{(\gamma-1) m}+\frac{\gamma T_{2} L_{2} M_{2}^{2}}{2 m}+\frac{1}{m} \sum_{j} Q_{j} f_{j}\left|=\frac{\gamma T_{1} L_{1}}{(\gamma-1) m}+\frac{\gamma T_{1} L_{1} M_{1}^{2}}{2 m}+\frac{1}{m} \sum_{j} Q_{j} f_{j}\right|_{1}
$$

where $L_{1}$ and $L_{2}$ are the parameter $L_{i}$, introduced in chapter 2 , evaluated upstream and downstream respectively. For $\gamma=5 / 3$ equation (E15) becomes:

$$
\left.\sum Q f_{1}\right\rfloor_{2}+2.5 T_{2} L_{2}\left(1+\frac{M_{2}^{2}}{3}\right)=\sum Q f_{j} L_{1}+2.5 T_{1} L_{1}\left(1+\frac{M_{1}^{2}}{3}\right)
$$

The continunity and momentum equations (E1) and (E2) remain unchanged for this case. Accordingly, equation (E10) can be written as:

$$
\frac{P_{2}}{P_{1}}=\frac{1+\gamma M_{1}^{2}}{1+\gamma M_{2}^{2}}=\frac{n_{2} T_{2} L_{2}}{n_{1} T_{1} L_{1}}
$$

Hence,

$$
\frac{T_{2} L_{2}}{T_{1} L_{1}}=\frac{n_{1}}{n_{2}} \frac{\left(1+\gamma M_{1}^{2}\right)}{\left(1+\gamma M_{2}^{2}\right)}
$$

but

$$
\frac{n_{1}}{n_{2}}=\frac{v_{2}}{v_{1}}=\frac{M_{2}}{M_{1}} \sqrt{\frac{T_{2} L_{2}}{T_{1} L_{1}}}
$$

Thus, equation (E17) becomes: 


$$
\frac{T_{2} L_{2}}{T_{1} L_{1}}=\left(\frac{M_{2}}{M_{1}}\right)^{2} \frac{\left(1+\gamma M_{1}^{2}\right)^{2}}{\left(1+\gamma M_{2}^{2}\right)^{2}}
$$

If the downstream Mach number $\mathrm{M}_{2}$ is assumed, then temperature, and density are obtained by iterating on equations (E16) and (E18). To minimize the number of iterations, $M_{2}$ is calculated from equation (E11). 


\section{APPENDIX F}

\section{Critical Flow Model}

Recall the ablation cloud energy balance equation (3-3) can be written as:

$$
n v \frac{d}{d r}\left[\frac{\gamma T L_{i}}{(\gamma-1)}+m \frac{v^{2}}{2}+\sum_{j=1}^{6} Q f_{j}\right]=\frac{d q}{d r}
$$

The flow velocity, $v$, is assumed equal to the local sound speed i.e.,

$$
v=\left[\frac{\gamma T L_{i}}{m}\right]^{1 / 2}
$$

Inserting eqn. (F2) into (F1) gives after some arrangement,

$$
\frac{d\left(T L_{\nu}\right)}{d r}=\frac{2(\gamma-1)}{\gamma(\gamma+1)}\left[\frac{1}{n v} \frac{d q}{d r}-\frac{d}{d r} \sum_{j=1}^{6} Q f_{j}\right]
$$

Using the dimensionless quantities (introduced in chapter 3), eqn. (F3) becomes

$$
\frac{d(\theta L)}{d r^{\prime}}=\left(\frac{2}{\gamma+1}\right) A_{*}\left[s-\frac{d}{d r^{\prime}} \sum Q f_{j}\right]
$$

from which we get after dropping the primes:

$$
L \frac{d \theta}{d r}=\left(\frac{2}{\gamma+1}\right) A_{*}\left[S-\frac{d}{d r} \sum Q f_{j}\right]-\theta \frac{d L}{d r}
$$

or 


$$
L \frac{d \theta}{d r}=\left(\frac{2}{\gamma+1}\right) A_{*}\left[S-\frac{d}{d r} \sum Q f_{j}\right]-\frac{\theta}{L_{*}} \sum j \frac{d f_{j}}{d r}
$$

The rate equations and the heat equation do change and are given by eqns. (5-20) and (5-21):

$$
\begin{aligned}
& \frac{d f_{j}}{d r}=\frac{n_{+} r_{*}}{v A} \sum_{j} f_{j}\left[f_{j-1} c_{j-1}^{i \alpha}-f_{j}\left(c_{j}^{\infty \alpha x}+\alpha_{j}^{\operatorname{cor}}\right)+f_{j+1} \alpha_{j+1}^{\infty \alpha x}\right] \\
& \frac{d q}{d r}=C_{*} G(u) / v A
\end{aligned}
$$

where all the variables have their usual definitions. 